\title{
On the origin of divergences in time-dependent orbifolds
}

\author{
Andrea Arduino $^{\mathrm{a}}$, Riccardo Finotello ${ }^{\mathrm{b}}$, Igor Pesando $^{\mathrm{c}}$ \\ Dipartimento di Fisica, I.N.F.N.-Sezione di Torino, Università di Torino, Via P. Giuria 1, 10125 Turin, Italy
}

Received: 26 March 2020 / Accepted: 2 May 2020 / Published online: 26 May 2020

(C) The Author(s) 2020

\begin{abstract}
We consider time-dependent orbifolds in String Theory and we show that divergences are not associated with a gravitational backreaction since they appear in the open string sector too. They are related to the non existence of the underlying effective field theory as in several cases fourth and higher order contact terms do not exist. Since contact terms may arise from the exchange of string massive states, we investigate and show that some three points amplitudes with one massive state in the open string sector are divergent on the time-dependent orbifolds. To check that divergences are associated with the existence of a discrete zero eigenvalue of the Laplacian of the subspace with vanishing volume, we construct the Generalized Null Boost Orbifold where this phenomenon can be turned on and off.
\end{abstract}

\section{Contents}

1 Introduction and conclusion . . . . . . . . . . . 1

2 Scalar QED on NBOand divergences . . . . . . . 3

2.1 Geometric preliminaries ........ 3

2.2 Free scalar action . . . . . . . . . . . . . 4

2.3 Free photon action ......... 5

2.4 Cubic interaction .......... . . 6

2.5 Quartic interactions and divergences ... . . 7

2.6 Failure of obvious divergence regularizations . 7

2.7 A hope from twisted state background . . . . 8

3 NBOeigenfunctions from covering space . . . . . 8

3.1 Spin 0 wave function from Minkowski space . . 9

3.2 Spin 1 wave function from Minkowski space . . 9

3.3 Tensor wave function from Minkowski space . 11

4 Overlap of wave functions and their derivatives . . . 13

4.1 Overlaps without derivatives . . . . . . . . 13

4.2 An overlap with one derivative . . . . . . . . 13

\footnotetext{
a e-mail: andrea.arduino@to.infn.it

b e-mail: riccardo.finotello@to.infn.it

c e-mail: ipesando@to.infn.it (corresponding author)
}

4.3 An overlap with two derivatives . . . . . . . 14

5 String three points amplitudes with one massive state 15

5.1 Level 2 massive state . . . . . . . . . . . 15

5.2 Two tachyons first massive state amplitude . . . 16

6 Scalar QED on GNBOand divergences . . . . . . . 16

6.1 Geometric preliminaries . . . . . . . . . 16

6.2 Free scalar field . . . . . . . . . . . . 17

6.3 Free photon action . . . . . . . . . . 18

6.4 Cubic interaction ............ . . 20

6.5 Quartic interactions ............ 20

6.6 Resurgence of divergences . . . . . . . . . 21

6.7 A comment on the null brane regularization . . 21

7 Quick analysis of the BO ........... . . 21

7.1 Geometric preliminaries ......... 22

7.2 Free scalar action . . . . . . . . . . . . 22

7.3 Eigenmodes on BOfrom covering space . . . . 22 7.3 .1 Spin 0 . . . . . . . . . . . . . . 22

7.3 .2 Spin $2 \ldots . . . . . . . . . .23$

7.4 Overlap of wave functions and their derivatives and a divergent three points String Amplitude . 25

7.4.1 Overlaps without derivatives . . . . . . 25

7.4.2 An overlap with two derivatives . . . . . 25

A Complete tensor wave function in the NBO . . . . . 26

B Complete overlap with two derivatives in the NBO . 27

References ................ 27

\section{Introduction and conclusion}

String Theory is often considered to be one, if not the best, candidate to describe quantum gravity and therefore the Big Bang singularity. Unfortunately and puzzlingly, the first attempts to consider space-like [1] or light-like singularities $[2,3]$ by means of orbifold techniques yielded divergent four points closed string amplitudes (see $[4,5]$ for reviews). This is somewhat embarrassing for a theory touted as a theory of quantum gravity. The aim of this paper is to elucidate the origin of these singularities in amplitudes. 
A number of reasons are cited in the literature for the existence of these singularities. The most widespread is that they are due to large backreaction of the incoming matter into the singularity due to the exchange of a single graviton [6,7]. This claim was already questioned in the literature where the $O$-plane orbifold was constructed. This orbifold should be stable against the gravitational collapse but it exhibits divergences in the amplitudes (see the discussion in [4]). We will show in a more direct way that this claim is false.

What has gone unnoticed is that in the Null Boost Orbifold (NBO) [2] even the four open string tachyons amplitude is divergent. Since we are working at tree level this means that gravity is not the problem. In fact in Eq. (6.16) of [2], the four tachyons amplitude in the divergent region reads

$A_{4} \sim \int_{q \sim \infty} \frac{\mathrm{d} q}{|q|} A(q) \quad$ with

$A_{\text {closed }} \sim q^{\alpha^{\prime}\left(\frac{4}{\alpha^{\prime}}-\vec{p}_{\perp t}^{2}\right)}$ and

$A_{\text {open }} \sim q^{\alpha^{\prime}\left(\frac{1}{\alpha^{\prime}}-\vec{p}_{\perp t}^{2}\right)} \operatorname{tr}\left(\left\{T_{1}, T_{2}\right\}\left\{T_{3}, T_{4}\right\}\right)$,

where $T$ are the Chan-Paton matrices. Moreover divergences in string amplitudes are not limited to four points: interestingly we show that the open string three point amplitude with two tachyons and the first massive state may be divergent when some physical polarization are chosen.

The true problem is therefore not related to a gravitational issue, but to the non existence of the effective field theory. In fact when we express the theory using the eigenmodes of the kinetic terms some coefficients do not exist, even as a distribution. This is true for both open and closed string sectors since it manifests also in the four scalar contact term. This problem can be roughly traced back to the vanishing volume of a subspace and the existence of a discrete zero mode of the Laplacian on this subspace.

In Sect. 2 in order to elucidate the problem of singularities in open string we start by considering the NBOwhere we try to construct a scalar QED theory. However, even the four scalars vertex is ill defined.

Divergences in scalar QED are due to the behaviour of the eigenfunctions of the scalar d'Alembertian near the singularity but in a somehow unexpected way. Near the singularity $u=0$ all but one eigenfunctions behave as $\frac{1}{\sqrt{|u|}} e^{i \frac{\mathcal{A}}{u}}$ with $\mathcal{A} \neq 0$. The product of $N$ eigenfunctions gives a singularity $|u|^{-N / 2}$ which is technically not integrable. However the exponential term $e^{i \frac{\mathcal{A}}{u}}$ allows for an interpretation as distribution when $\mathcal{A}=0$ is not an isolated point. When $\mathcal{A}=0$ is isolated the singularity is definitely not integrable and there is no obvious interpretation as a distribution. Specifically in the $\mathrm{NBO} \mathcal{A} \sim \frac{l^{2}}{k_{+}}$with $l$ the momentum along the compact direction therefore there is one eigenfunction with isolated $\mathcal{A}=0$ : the one which is associated with the discrete momen- tum $l=0$ along the orbifold compact direction. It is the eigenfunction which is constant along that direction and it is the root of all divergences. If the direction were not compact or there were at least one another non compact direction contributing to $\mathcal{A}$ we could avoid the problem.

We then check whether the most obvious ways of regularizing the theory by making $\mathcal{A}$ not vanishing may work. The first regularization we try is to use a Wilson line along the compact direction. It works for the scalar QED and almost for string theory but not completely. The diverging three point string amplitude involves an anti-commutator of the ChanPaton factor therefore it is divergent also for a neutral string, i.e. for a string with both ends attached to the same brane. This kind of string does not feel Wilson lines. Moreover anticommutators are present in amplitudes with massive states in unoriented and supersymmetric strings and therefore neither worldsheet parity nor supersymmetry help. The second obvious regularization is to use higher derivatives couplings to Ricci tensor which is the only non vanishing tensor associated with the (regularized) metric. Unfortunately if we assume that the parameter entering the metric regularization is of the order of the string scale these terms do not help. In any case it seems that a sensible regularization must couple to all open string in the same way and this suggests a gravitational coupling. Therefore we then give a cursory look to whether closed string winding modes could help [8], as already suggested in $[1,3]$ in analogy with the resolution of static singularities. Twisted closed strings become massless near the singularity and they should in some way be included. They generate a background potential $B_{\mu \nu}$ which is equivalent to a electromagnetic background from the open string perspective. Under a plausible modification of the scalar action which is suggested by the two tachyons - two photons amplitude the problems seem to be solvable.

In any case we now understand the origin of the string divergences from the point of view of non existence of contact terms in the effective field theory. String theory divergences come from ill defined contact terms which must be reproduced by string amplitudes. In the effective field theory contact terms arise from String Theory also through the exchange of massive string states and this suggests to examine three point amplitudes with one massive state.

To do so, even if not strictly necessary to show the existence of divergences, we want to understand how to write the polarizations for the massive state on orbifold from the Minkowski ones. This is tackled in Sect. 3.

In Sect. 4 we consider overlaps of different wave functions and derivatives thereof. These overlaps are related in fact to the coefficients of the expansions of the effective theory in eigenfunctions of the kinetic terms and therefore they are strictly related to string amplitudes on orbifolds.

In Sect. 5 we go back to String Theory and we use the result of the previous section in order to verify that in the NBOthe 
open string three points amplitude with two tachyons and one first level massive string state does indeed diverge when some physical polarization are chosen. An intuitive reason is that we have an infinite number of images and the delta functions associated with momentum conservation have an accumulation point of their support. Nevertheless the existence of the accumulation point is not sufficient since three tachyons amplitude converges: the coefficients of the deltas matter too, and the convergence must be verified.

As stated above, if the directions with vanishing volume were not compact or a "mixture" of compact ones and at least one non compact we could avoid the divergences. To check this point, in Sect. 6 we introduce the Generalized Null Boost Orbifold (GNBO) as a generalization of the NBOwhich still has a light-like singularity and is generated by one Killing vector. However in this model there are two directions associated with $\mathcal{A}$, one compact and one non compact. We can then construct the scalar QED and the effective field theory which extends it with the inclusion of higher order terms since all terms have a distributional interpretation. However if a second Killing vector is used to compactify the formerly non compact direction, the theory has again the same problems as in the NBOcase. In the literature there are also other attempts at regularizing the NBOsuch as the Null Brane. This kind of orbifold was originally defined in $[9,10]$ and studied in perturbation theory in [3]. This orbifold shares with the GNBOthe fact that there is a non compact direction as discussed in this section. In this case it is indeed possible to build single particle wave functions which leads to the convergence of the smeared amplitudes on the Null Brane.

In Sect. 7 we then quickly examine the Boost Orbifold (BO) where the divergences are generically milder [11,12]. The scalar eigenfunctions generically behave as $|t|^{ \pm i \frac{l}{\Delta}}$ near the singularity but there is one eigenfunction which behaves as $\log (|t|)$ and again it is the constant eigenfunction along the compact direction which is the origin of all divergences. In particular the scalar QED can be defined and the first term which gives a divergent contribution is of the form $|\phi \dot{\phi}|^{2}$, i.e divergences are hidden into the derivative expansion of the effective field theory. Again three points open string amplitudes with one massive state diverge.

The lessons to be learnt from these examples are many.

First, it seems that String Theory cannot do better than field theory when the latter does not exist, at least at the perturbative level where one deals with particles.

Second, when spacetime becomes singular, the string massive modes are not anymore spectators.

Third and related to the last point, the previous problems seem to suggest that issues with spacetime singularities are hidden into contact terms and interactions with massive states. This would explain in an intuitive way why the eikonal approach to gravitational scattering seems to work well: eikonal is indeed concerned with three point massless interactions. In fact it appears [13] that the classical and quantum scattering on an electromagnetic wave [14] or gravitational wave [15] in BOand NBOare well behaved. From this point of view the ACV approach [16,17], especially when considering massive external states [18], may be more sensible.

Finally it seems that all issues are related with what happens to the Laplacian associated with the spacelike subspace with vanishing volume at the singularity. If there is a discrete zero eigenvalue the theory develops divergences.

\section{Scalar QED on NBOand divergences}

As we discussed in the Introduction, the four open string tachyons amplitude diverges in the NBO Given the suggestion in the literature [4] that this can be cured by the eikonal resummation, we would like to consider the scalar QED on the NBO Another reason is that all eigenmodes can be written using elementary functions thus making the issues more transparent. Its action is given by

$$
\begin{aligned}
& \mathrm{S}_{S Q E D}=\int_{\Omega} \mathrm{d}^{D} x \sqrt{-\operatorname{det} g} \\
& \quad \times\left(-\left(D^{\mu} \phi\right)^{*} D_{\mu} \phi-M^{2} \phi^{*} \phi-\frac{1}{4} f^{\mu \nu} f_{\mu \nu}-\frac{g_{4}}{4}|\phi|^{4}\right),
\end{aligned}
$$

with

$D_{\mu} \phi=\left(\partial_{\mu}-i\right.$ e $\left.a_{\mu}\right) \phi, \quad f_{\mu \nu}=\partial_{\mu} a_{\nu}-\partial_{\nu} a_{\mu}$.

We reserve small letters for quantities defined on the orbifold and capital ones for those defined in Minkowski. Moreover $\Omega$ denotes the orbifold. We will construct directly both the scalar and the spin-1 eigenfunctions which we can use as a starting point for the perturbative computations.

\subsection{Geometric preliminaries}

In $\mathbb{R}^{1, D-1}$ with coordinates $\left(x^{\mu}\right)=\left(x^{+}, x^{-}, x^{2}, \vec{x}\right)$ and metric

$\mathrm{d} s^{2}=-2 \mathrm{~d} x^{+} \mathrm{d} x^{-}+\left(\mathrm{d} x^{2}\right)^{2}+\eta_{i j} \mathrm{~d} x^{i} \mathrm{~d} x^{j}$,

we consider the following change of coordinates to $\left(x^{\alpha}\right)=$ $(u, v, z, \vec{x})$

$\left\{\begin{array}{l}x^{-}=u \\ x^{2}=\Delta u z \\ x^{+}=v+\frac{1}{2} \Delta^{2} u z^{2}\end{array} \Leftrightarrow\left\{\begin{array}{l}u=x^{-} \\ z=\frac{x^{2}}{\Delta x^{-}} \\ v=x^{+}-\frac{1}{2} \frac{\left(x^{2}\right)^{2}}{x^{-}}\end{array}\right.\right.$

Then the metric becomes:

$\mathrm{d} s^{2}=-2 \mathrm{~d} u \mathrm{~d} v+(\Delta u)^{2}(\mathrm{~d} z)^{2}+\eta_{i j} \mathrm{~d} x^{i} \mathrm{~d} x^{j}$, 
along with the non vanishing geometrical quantities

$$
-\operatorname{det} g=(\Delta u)^{2},
$$

and

$\Gamma_{z z}^{v}=\Delta^{2} u, \quad \Gamma_{u z}^{z}=\frac{1}{u}$.

Riemann and Ricci tensor components however vanish since at this stage we only performed a change of coordinates from the original Minkowski spacetime: locally it is the same as the NBOand they must have the same local differential geometry.

The NBOis introduced by identifying points along the orbits of the Killing vector:

$$
\begin{aligned}
\kappa & =-i(2 \pi \Delta) J_{+2} \\
& =(2 \pi \Delta)\left(x^{2} \partial_{+}+x^{-} \partial_{2}\right) \\
& =2 \pi \partial_{z},
\end{aligned}
$$

in such a way that

$x^{\mu} \equiv \mathcal{K}^{n} x^{\mu}, \quad n \in \mathbb{Z}$,

where $\mathcal{K}^{n}=e^{n \kappa}$, leads to the identifications

$$
\begin{aligned}
x & =\left(\begin{array}{c}
x^{-} \\
x^{2} \\
x^{+} \\
\vec{x}
\end{array}\right) \equiv \mathcal{K}^{n} x \\
& =\left(\begin{array}{c}
x^{-} \\
x^{2}+n(2 \pi \Delta) x^{-} \\
x^{+}+n(2 \pi \Delta) x^{2}+\frac{1}{2} n^{2}(2 \pi \Delta)^{2} x^{-} \\
\vec{x}
\end{array}\right)
\end{aligned}
$$

or to

$(u, v, z, \vec{x}) \equiv(u, v, z+2 \pi n, \vec{x})$

in the coordinates $\left(x^{\alpha}\right)$ where $\kappa=2 \pi \partial_{z}$ is a global Killing vector.

For future use in Sect. 2.6, we notice that we could regularize the metric as

$\mathrm{d} s^{2}=-2 \mathrm{~d} u \mathrm{~d} v+\Delta^{2}\left(u^{2}+\epsilon^{2}\right)(\mathrm{d} z)^{2}+\eta_{i j} \mathrm{~d} x^{i} \mathrm{~d} x^{j}$.

Then the non vanishing geometrical quantities are

$-\operatorname{det} g=\Delta^{2}\left(u^{2}+\epsilon^{2}\right)$,

and

$\Gamma_{z z}^{v}=\Delta^{2} u, \quad \Gamma_{u z}^{z}=\frac{u}{u^{2}+\epsilon^{2}}$,

which lead to the following Riemann and Ricci tensor components:

$$
\begin{aligned}
R_{u z u}^{z} & =-\frac{\epsilon^{2}}{\left(u^{2}+\epsilon^{2}\right)^{2}}, \quad R_{z z u}^{v}=-\frac{\Delta^{2} \epsilon^{2}}{u^{2}+\epsilon^{2}}, \\
R i c_{u u} & =-\frac{\epsilon^{2}}{\left(u^{2}+\epsilon^{2}\right)^{2}} .
\end{aligned}
$$

Since $\delta_{\text {reg }}(u)=\frac{1}{\pi} \frac{\epsilon}{u^{2}+\epsilon^{2}}$ this means that $R_{u z u}^{z} \sim\left[\delta_{r e g}(u)\right]^{2}$.

\subsection{Free scalar action}

We now want to find the eigenmodes of the Laplacian in order to write in a diagonal way the scalar kinetic term given by ${ }^{1}$

$$
\begin{aligned}
& \text { S scalar kin } \\
& =\int_{\Omega} \mathrm{d}^{D} x \sqrt{-\operatorname{det} g}\left(-g^{\alpha \beta} \partial_{\alpha} \phi^{*} \partial_{\beta} \phi-M^{2} \phi^{*} \phi\right) \\
& =\int \mathrm{d}^{D-3} \vec{x} \int \mathrm{d} u \int \mathrm{d} v \int_{0}^{2 \pi} \mathrm{d} z|\Delta u| \\
& \quad \times\left(\partial_{u} \phi^{*} \partial_{v} \phi+\partial_{v} \phi^{*} \partial_{u} \phi-\frac{1}{(\Delta u)^{2}} \partial_{z} \phi^{*} \partial_{z} \phi\right. \\
& \left.\quad-\partial_{i} \phi^{*} \partial_{i} \phi-M^{2} \phi^{*} \phi\right) .
\end{aligned}
$$

The solution to the equation of motion is enough when we want to perform the canonical quantization. Since we want to use the Feynman diagrams, we consider the path integral approach: we take off-shell modes and solve the eigenvalue problem $\square \phi_{r}=r \phi_{r}$. By comparing with the flat case we see that $r$ is $2 k_{-} k_{+}-\vec{k}^{2}$ when $k$ is the flat coordinates momentum. We therefore have

$-2 \partial_{u} \partial_{v} \phi_{r}-\frac{1}{u} \partial_{v} \phi_{r}+\frac{1}{(\Delta u)^{2}} \partial_{z}^{2} \phi_{r}+\partial_{i}^{2} \phi_{r}=r \phi_{r}$.

Using Fourier transforms, it then easily follows that the eigenmodes are

$\phi_{\left\{k_{+} l \vec{k} r\right\}}(u, v, z, \vec{x})=e^{i k_{+} v+i l z+i \vec{k} \cdot \vec{x}} \tilde{\phi}_{\left\{k_{+} l \vec{k} r\right\}}(u)$,

with

$\tilde{\phi}_{\left\{k_{+} \mid \vec{k} r\right\}}(u)=\frac{1}{\sqrt{(2 \pi)^{D} 2\left|\Delta k_{+}\right||u|}} e^{-i \frac{l^{2}}{2 \Delta^{2} k_{+}} \frac{1}{u}+i \frac{\vec{k}^{2}+r}{2 k_{+}} u}$,

and

$\phi_{\left\{k_{+} l \vec{k} r\right\}}^{*}(u, v, z, \vec{x})=\phi_{\left\{-k_{+}-l-\vec{k} r\right\}}(u, v, z, \vec{x})$,

$\overline{1}^{1}$ The factor $-g^{\alpha \beta}$ is due to the choice of the East coast convention for the metric, i.e.:

$-g^{\alpha \beta} \partial_{\alpha} \phi^{*} \partial_{\beta} \phi-M^{2} \phi^{*} \phi \sim+|\dot{\phi}|^{2}-M^{2}|\phi|^{2} \sim \mathrm{E}^{2}-M^{2}$. 
where we have chosen the numeric factor in order to get a canonical normalization:

$$
\begin{aligned}
& \left(\phi_{\left\{k_{(1)+} l_{(1)} \vec{k}_{(1)} r_{(1)}\right\}}, \phi_{\left\{k_{(2)+} l_{(2)} \vec{k}_{(2)} r_{(2)\}}\right)}\right. \\
& =\int \mathrm{d}^{D-3} \vec{x} \int \mathrm{d} u \int \mathrm{d} v \\
& \quad \times \int_{0}^{2 \pi} \mathrm{d} z|\Delta u| \phi_{\left\{k_{(1)+} l_{(1)} \vec{k}_{(1)} r_{(1)}\right\}} \phi_{\left\{k_{(2)+} l_{(2)} \vec{k}_{(2)} r_{(2)}\right\}} \\
& =\delta^{D-3}\left(\vec{k}_{(1)}+\vec{k}_{(2)}\right) \delta\left(r_{(1)}-r_{(2)}\right) \\
& \quad \times \delta\left(k_{(1)+}+k_{(2)+}\right) \delta_{l_{(1)},-l_{(2)}} .
\end{aligned}
$$

We can then perform the off-shell expansion

$$
\begin{aligned}
\phi(u, v, z, \vec{x})= & \int \mathrm{d}^{D-3} \vec{k} \int \mathrm{d} r \int \mathrm{d} k_{+} \\
& \times \sum_{l \in \mathbb{Z}} \mathcal{A}_{\left\{k_{+} l \vec{k} r\right\}} \phi_{\left\{k_{+} l \vec{k} r\right\}}(u, v, z, \vec{x}),
\end{aligned}
$$

so that the scalar kinetic term becomes

$$
\begin{aligned}
\mathrm{S}_{\text {scalar kin }}=\int & \mathrm{d}^{D-3} \vec{k} \int \mathrm{d} r \int \mathrm{d} k_{+} \\
& \times \sum_{l \in \mathbb{Z}}\left(r-M^{2}\right) \mathcal{A}_{\left\{k_{+} l \vec{k} r\right\}} \mathcal{A}_{\left\{k_{+} l \vec{k} r\right\}}^{*}
\end{aligned}
$$

\subsection{Free photon action}

The photon action can be written as

$$
\begin{aligned}
\mathrm{S}_{\text {spin-1 kin }}= & \int_{\Omega} \mathrm{d}^{D} x \sqrt{-\operatorname{det} g} \\
& \times\left(-\frac{1}{2} g^{\alpha \beta} g^{\gamma \delta} D_{\alpha} a_{\gamma}\left(D_{\beta} a_{\delta}-D_{\delta} a_{\beta}\right)\right) .
\end{aligned}
$$

If we choose the Lorenz gauge ${ }^{2}$

$$
\begin{aligned}
D^{\alpha} a_{\alpha}= & -\frac{1}{u} a_{v}-\partial_{u} a_{v}-\partial_{v} a_{u}+\frac{1}{\Delta^{2} u^{2}} \partial_{z} a_{z} \\
& +\eta^{i j} \partial_{i} a_{j}=0
\end{aligned}
$$

and remember that covariant derivatives commute since we are locally flat, the equations of motion read $(\square a)_{\alpha}=0$.

\footnotetext{
2 Indeed it is exactly the usual Lorenz gauge since locally the space is Minkowski.
}

Explicitly we have:

$$
\begin{aligned}
(\square a)_{u}= & \frac{1}{u^{2}} a_{v}-\frac{2}{\Delta^{2} u^{3}} \partial_{z} a_{z} \\
& +\left[-2 \partial_{u} \partial_{v}-\frac{1}{u} \partial_{v}+\frac{1}{\Delta^{2} u^{2}} \partial_{z}^{2}+\eta^{i j} \partial_{i} \partial_{j}\right] a_{u}, \\
(\square a)_{v}= & {\left[-2 \partial_{u} \partial_{v}-\frac{1}{u} \partial_{v}+\frac{1}{\Delta^{2} u^{2}} \partial_{z}^{2}+\eta^{i j} \partial_{i} \partial_{j}\right] a_{v}, } \\
(\square a)_{z}= & -\frac{2}{u} \partial_{z} a_{v} \\
& +\left[-2 \partial_{u} \partial_{v}+\frac{1}{u} \partial_{v}+\frac{1}{\Delta^{2} u^{2}} \partial_{z}^{2}+\eta^{i j} \partial_{i} \partial_{j}\right] a_{z}, \\
(\square a)_{i}= & {\left[-2 \partial_{u} \partial_{v}-\frac{1}{u} \partial_{v}+\frac{1}{\Delta^{2} u^{2}} \partial_{z}^{2}+\eta^{i j} \partial_{i} \partial_{j}\right] a_{i} . }
\end{aligned}
$$

As in the scalar case we are actually interested in solving the eigenmodes problem $(\square a)_{\alpha}=r a_{\alpha}$. We proceed hierarchically: first we solve for $a_{v}$ and $a_{i}$ whose equations are the same as the one for the scalar field, then we insert the solutions as a source in the equation ${ }^{3}$ for $a_{z}$ and eventually we solve for $a_{u}$. We get the solutions:

$$
\begin{aligned}
& \left\|\tilde{a}_{\left\{k_{+} l \vec{k} r\right\} \alpha}(u)\right\|=\left(\begin{array}{c}
\tilde{a}_{u} \\
\tilde{a}_{v} \\
\tilde{a}_{z} \\
\tilde{a}_{i}
\end{array}\right) \\
& =\sum_{\underline{\alpha} \in\{\underline{u}, \underline{v}, \underline{z}, \underline{i}\}} \mathcal{E}_{\left\{k_{+} l \vec{k} r\right\} \underline{\alpha}}\left\|\tilde{a}_{\left\{k_{+} l \vec{k} r\right\} \alpha}^{\underline{\alpha}}(u)\right\| \\
& =\mathcal{E}_{\left\{k_{+} l \vec{k} r\right\} \underline{u}}\left(\begin{array}{c}
1 \\
0 \\
0 \\
0
\end{array}\right) \tilde{\phi}_{\left\{k_{+} l \vec{k} r\right\}}(u)
\end{aligned}
$$

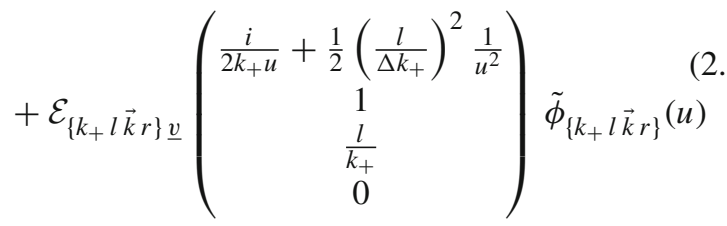

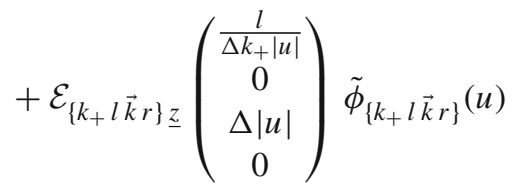

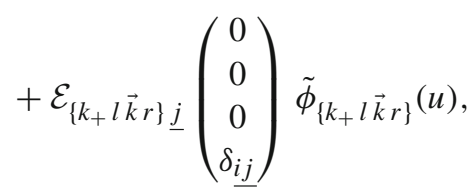

${ }^{3}$ Notice that inside the square brackets of the differential equation for $a_{z}$ there is a different sign for the term $\frac{1}{u} \partial_{v}$ with respect to the equation for the scalar field. 
then we can expand the off-shell fields as

$$
\begin{aligned}
& a_{\alpha}(u, v, z, \vec{x})=\int \mathrm{d}^{D-3} \vec{k} \int \mathrm{d} r \int \mathrm{d} k_{+} \\
& \sum_{l \in \mathbb{Z}} \sum_{\alpha \in\{\underline{u}, \underline{v}, \underline{z}, \underline{i}\}} \mathcal{E}_{\left\{k_{+} l \vec{k} r\right\} \underline{\alpha}} a_{\left\{k_{+} l \vec{k} r\right\} \alpha}^{\underline{\alpha}}(u, v, z, \vec{x}),
\end{aligned}
$$

where $a_{\left\{k_{+} l \vec{k} r\right\} \alpha}(u, v, z, \vec{x})=\tilde{a}_{\left\{k_{+} l \vec{k} r\right\} \alpha}^{\underline{\alpha}}(u) e^{i\left(k_{+} v+l z+\vec{k} \cdot \vec{x}\right)}$.

We can also compute the normalization as

$$
\begin{aligned}
& \left(a_{(1)}, a_{(2)}\right)=\int \mathrm{d}^{D-3} \vec{x} \int \mathrm{d} u \int \mathrm{d} v \int_{0}^{2 \pi} \mathrm{d} z|\Delta u| \\
& \times\left(g^{\alpha \beta} a_{\left\{k_{(1)+} l_{(1)} \vec{k}_{(1)} r_{(1)}\right\} \alpha} a_{\left\{k_{(2)+} l_{(2)} \vec{k}_{(2)} r_{(2)} \beta\right.}\right) \\
& =\mathcal{E}_{\left\{k_{(1)+} l_{(1)} \vec{k}_{(1)} r_{(1)}\right.} \circ \mathcal{E}_{\left\{k_{(2)+} l_{(2)} \vec{k}_{(2)} r_{(2)}\right\}} \\
& \times \delta^{D-3}\left(\vec{k}_{(1)}+\vec{k}_{(2)}\right) \\
& \delta\left(r_{(1)}-r_{(2)}\right) \delta\left(k_{(1)+}+k_{(2)+}\right) \delta_{l_{(1)},-l_{(2)}} \text {, }
\end{aligned}
$$

with $^{4}$

$$
\begin{aligned}
\mathcal{E}_{(1)} \circ \mathcal{E}_{(2)}= & -\mathcal{E}_{(1) \underline{u}} \mathcal{E}_{(2) \underline{v}}-\mathcal{E}_{(1) \underline{v}} \mathcal{E}_{(2) \underline{u}} \\
& +\mathcal{E}_{(1) \underline{z}} \mathcal{E}_{(2) \underline{z}}+\eta \underline{i}-\mathcal{E}_{(1) \underline{i}} \mathcal{E}_{(2) \underline{j}} .
\end{aligned}
$$

Finally the Lorenz gauge reads

$$
\begin{gathered}
\eta^{i j} k_{i} \mathcal{E}_{\left\{k_{+} l \vec{k} r\right\} \underline{j}}-k_{+} \mathcal{E}_{\left\{k_{+} l \vec{k} r\right\} \underline{u}} \\
-\frac{\vec{k}^{2}+r}{2 k_{+}} \mathcal{E}_{\left\{k_{+} l \vec{k} r\right\}_{\underline{v}}}=0,
\end{gathered}
$$

which does not impose any constraint on the transverse polarization $\mathcal{E}_{\left\{k_{+} l \vec{k} r\right\} z}$, while the photon kinetic term becomes

$$
\begin{aligned}
\mathrm{S}_{\text {spin-1 kin }}= & \int \mathrm{d}^{D-3} \vec{k} \int \mathrm{d} r \int \mathrm{d} k_{+} \\
& \sum_{l \in \mathbb{Z}} \frac{1}{2} r \mathcal{E}_{\left\{k_{+} l \vec{k} r\right\}} \circ \mathcal{E}_{\left\{k_{+} l \vec{k} r\right\}}^{*} .
\end{aligned}
$$

\subsection{Cubic interaction}

With the definition of the d'Alembertian eigenmodes we can now examine the cubic vertex which reads

$$
\begin{gathered}
\mathrm{S}_{\text {cubic }}=\int_{\Omega} \mathrm{d}^{D} x \sqrt{-\operatorname{det} g}\left(-i e g^{\alpha \beta}\right. \\
\left.a_{\alpha}\left(\phi^{*} \partial_{\beta} \phi-\partial_{\beta} \phi^{*} \phi\right)\right) .
\end{gathered}
$$

\footnotetext{
${ }^{4}$ We use a shortened version of the polarizations $\mathcal{E}$ for the sake of readability. Specifically we write $\mathcal{E}_{(n) \underline{\alpha}}=\mathcal{E}_{\left\{k_{(n)+} l_{(n)} \vec{k}_{(n)} r_{(n)}\right\} \underline{\alpha}}$ thus hiding the understood dependence of the components of $\mathcal{E}_{(n)}$ on the momenta.
}

Its computation involves integrals such as

$$
\begin{aligned}
& \int \mathrm{d} u|\Delta u|\left(\frac{l}{u}\right)^{2} \prod_{i=1}^{3} \tilde{\phi}_{\left\{k_{(i)+} l_{(i)} \vec{k}_{(i)} r_{(i)}\right\}} \\
& \sim \int_{u \sim 0} \mathrm{~d} u\left(\frac{l^{2}}{|u|^{5 / 2}}\right) e^{-i \sum_{i=1}^{3} \frac{l_{(i)}{ }^{2}}{2 \Delta^{2} k_{(i)+}} \frac{1}{u}},
\end{aligned}
$$

and

$$
\begin{aligned}
& \int \mathrm{d} u|\Delta u|\left(\frac{1}{u}\right) \prod_{i=1}^{3} \tilde{\phi}_{\left\{k_{(i)+} l_{(i)} \vec{k}_{(i)} r_{(i)}\right\}} \\
& \sim \int_{u \sim 0} \mathrm{~d} u\left(\frac{1}{u|u|^{1 / 2}}\right) e^{-i \sum_{i=1}^{3} \frac{l_{(i)}^{2}}{2 \Delta^{2} k_{(i)+}} \frac{1}{u}},
\end{aligned}
$$

which can be interpreted as hints that the theory may be troublesome. The first integral would diverge if the factor $e^{i \frac{\mathcal{A}}{u}}$ were equal 1 . Fortunately it happens when all $l_{(*)}=0$ but in this case the integral vanishes (if we set $l_{(*)}=0$ before its evaluation). This however suggests that when all $l_{(*)}=0$, i.e. when the eigenfunctions are constant along the compact direction $z$, something is happening. On the other side when at least one $l$ is different from zero we have an integral such as

$\int_{u \sim 0} \mathrm{~d} u|u|^{-v} e^{i \frac{\mathcal{A}}{u}} \sim \int_{t \sim \infty} \mathrm{d} t t^{\nu-2} e^{i \mathcal{A} t}$.

All $l_{(*)}$ are discrete but $k_{(*)+}$ are not, therefore $\mathcal{A}$ has an isolated zero but otherwise it has continuous value and may be given a distributional meaning, similar to a derivative of the $\delta$.

The second integral has again issues when all $l_{(*)}=0$ and since it is not proportional to any $l$ as it stands it is divergent unless we take a principal part regularization which may be meaningful.

With all these warnings we can give anyhow meaning to the cubic terms and we get ${ }^{5}$

$$
\begin{aligned}
& \mathrm{S}_{\text {cubic }}=\prod_{i=1}^{3}\left[\int \mathrm{d}^{D-3} \vec{k}_{(i)} \mathrm{d} r_{(i)} \mathrm{d} k_{(i)}+\sum_{l_{(i)}}\right] \\
& \times(2 \pi)^{D-1} \delta\left(\sum \vec{k}_{(i)}\right) \delta\left(\sum k_{(i)+}\right) \\
& \times \delta_{\left(\sum l_{(i)}\right)} e\left(\mathcal{A}_{\left\{-k_{(2)+}-l_{(2)}-\vec{k}_{(2)} r_{(2)}\right\}}\right)^{*} \mathcal{A}_{\left\{k_{(3)+} l_{(3)} \vec{k}_{(3)} r_{(3)}\right\}} \\
& \times\left\{\mathcal{E}_{\left\{k_{(1)+} l_{(1)} \vec{k}_{(1)} r_{(1)}\right\} \underline{u}} k_{(2)+} \mathcal{I}_{\{3\}}^{[0]}\right. \\
& +\mathcal{E}_{\left\{k_{(1)+} l_{(1)} \vec{k}_{(1)} r_{(1)}\right\} \underline{z}} \frac{k_{(2)+} l_{(1)}-l_{(2)} k_{(1)+}}{\Delta k_{(1)+}} \mathcal{J}_{(3)}^{[-1]}
\end{aligned}
$$

5 The notation (2) $\rightarrow$ (3) means that all previous terms inside the curly brackets appear again in exactly the same structure but with momenta of particle (3) in place of those of particle (2). 


$$
\begin{aligned}
& \left.+\mathcal{E}_{\left\{k_{(1)}+\right.} l_{(1)} \vec{k}_{(1)} r_{(1)}\right\} \underline{v} \\
& \times\left[\frac{\vec{k}_{(2)}^{2}+r_{(2)}}{2 k_{(2)+}} \mathcal{I}_{\{3\}}^{[0]}+i \frac{k_{(2)+}}{2 k_{(1)+}} \mathcal{I}_{\{3\}}^{[-1]}\right. \\
& \left.+\frac{1}{2} \frac{k_{(2)+}}{\Delta^{2}}\left(\frac{l_{(1)}}{k_{(1)+}}-\frac{l_{(2)}}{k_{(2)+}}\right)^{2} \mathcal{I}_{\{3\}}^{[-2]}\right]
\end{aligned}
$$

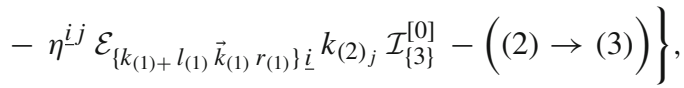

where we have defined also for future use

$$
\begin{aligned}
\mathcal{I}_{(1) \ldots(N)}^{[\nu]} & =\mathcal{I}_{\{N\}}^{[v]} \\
& \left.=\int_{-\infty}^{+\infty} \mathrm{d} u|\Delta u| u^{\nu} \prod_{i=1}^{N} \tilde{\phi}_{\left\{k_{(i)+} l_{(i)}\right.} \vec{k}_{(i)} r_{(i)}\right\} \\
& =\int_{-\infty}^{+\infty} \mathrm{d} u|\Delta u| u^{\nu} \prod_{i=1}^{N} \tilde{\phi}_{(i)}, \\
\mathcal{J}_{(N)}^{[\nu]} & =\int_{-\infty}^{+\infty} \mathrm{d} u|\Delta||u|^{\nu+1} \prod_{i=1}^{N} \tilde{\phi}_{\left\{k_{(i)+} l_{(i)} \vec{k}_{(i)} r_{(i)}\right\}},
\end{aligned}
$$

where $\tilde{\phi}_{(i)}=\tilde{\phi}_{\left\{k_{(i)+} l_{(i)} \vec{k}_{(i)} r_{(i)}\right\}}$ and $\tilde{\phi}_{(i)}=\tilde{\phi}_{\left\{k_{(i)+} l_{(i)} \vec{k}_{(i)} r_{(i)}\right\}}$ which will be used when not causing confusion.

\subsection{Quartic interactions and divergences}

In the previous section we have seen that the theory may have issues when all $l=0$, i.e. with eigenfunctions independent of the compact direction $z$ because some integrals were on the verge of diverging. The divergence issue will appear in a clear and unavoidable way when considering the quartic terms:

$$
\begin{aligned}
\mathrm{S}_{\text {quartic }}= & \int_{\Omega} \mathrm{d}^{D} x \sqrt{-\operatorname{det} g} \\
& \times\left(e^{2} g^{\mu v} a_{\mu} a_{v}|\phi|^{2}-\frac{g_{4}}{4}|\phi|^{4}\right),
\end{aligned}
$$

which can be expressed using the modes as

$$
\begin{aligned}
& \mathrm{S}_{\text {quartic }}=\prod_{i=1}^{4}\left[\int \mathrm{d}^{D-3} \vec{k}_{(i)} \mathrm{d} k_{(i)+} \mathrm{d} r_{(i)} \sum_{l_{(i)}}\right] \\
& \times(2 \pi)^{D-1} \delta\left(\sum \vec{k}_{(i)}\right) \delta\left(\sum k_{(i)+}\right) \delta_{\sum l_{(i)}, 0} \\
& \times\left\{e^{2}\left(\mathcal{A}_{\left\{-k_{(3)+}-l_{(3)}-\vec{k}_{(3)} r_{(3)}\right\}}\right)^{*} \mathcal{A}_{\left\{k_{(4)+} l_{(4)} \vec{k}_{(4)} r_{(4)}\right\}}\right. \\
& \times\left[\left(\mathcal{E}_{\left\{k_{(1)+} l_{(1)} \vec{k}_{(1)} r_{(1)\}}\right.} \circ \mathcal{E}_{\left\{k_{(2)+} l_{(2)} \vec{k}_{(2)} r_{(2)\}}\right.}\right) \mathcal{I}_{\{4\}}^{[0]}\right. \\
& -i \frac{1}{2} \mathcal{E}_{\left\{k_{(1)+} l_{(1)} \vec{k}_{(1)} r_{(1)}\right\} \underline{v}} \mathcal{E}_{\left\{k_{(2)+} l_{(2)} \vec{k}_{(2)} r_{(2)}\right\} \underline{v}} \\
& \times\left(\frac{1}{k_{(2)+}}+\frac{1}{k_{(1)+}}\right) \mathcal{I}_{\{4\}}^{[-1]}
\end{aligned}
$$

$$
\begin{aligned}
&+\frac{1}{2} \frac{\mathcal{E}_{\left\{k_{(1)+} l_{(1)} \vec{k}_{(1)} r_{(1)\}} \underline{\underline{v}}\right.} \mathcal{E}_{\left\{k_{(2)+} l_{(2)} \vec{k}_{(2)} r_{(2)} \underline{v}\right.}}{\Delta^{2}} \\
&\left.\times\left(\frac{l_{(1)}}{k_{(1)+}}-\frac{l_{(2)}}{k_{(2)+}}\right)^{2} \mathcal{I}_{\{4\}}^{[-2]}\right] \\
&- \frac{g_{4}}{4}\left(\mathcal{A}_{\left\{-k_{(1)+}-l_{(1)}-\vec{k}_{(1)} r_{(1)}\right\}}\right)^{*} \\
& \times\left(\mathcal{A}_{\left\{-k_{(2)+}-l_{(2)}-\vec{k}_{(2)} r_{(2)}\right)}\right)^{*} \\
& \mathcal{A}_{\left\{k_{(3)+} l_{(3)} \vec{k}_{(3)} r_{(3)}\right\}} \mathcal{A}_{\left\{k_{(4)+} l_{(4)} \vec{k}_{(4)} r_{(4)\}}\right.} \mathcal{I}_{\{4\}}^{[0]\} .}
\end{aligned}
$$

Now when setting $l_{(*)}=0$ all the surviving terms are divergent, explicitly $\mathcal{I}_{\{4\}}^{[0]} \sim \int d u|u|^{1-4 \times \frac{1}{2}}$ and $\mathcal{I}_{\{4\}}^{[-1]} \sim$ $\int d u|u|^{1-4 \times \frac{1}{2} \frac{1}{u}}$ since $\left.\tilde{\phi}\right|_{l=0} \sim|u|^{-\frac{1}{2}}$.

Obviously higher order terms in the effective field theory will behave even worse. This makes the theory ill defined and the string theory which should give this effective theory ill defined too.

\subsection{Failure of obvious divergence regularizations}

From the discussion in the previous section it is clear that the origin of the divergences is the sector $l=0$. When $l=$ 0 the highest order singularity of the Fourier transformed d'Alembertian equation vanishes. Explicitly we have:

$$
\begin{aligned}
& A \partial_{u} \tilde{\phi}_{\left\{k_{+} l \vec{k} r\right\}}+B(u) \tilde{\phi}_{\left\{k_{+} l \vec{k} r\right\}} \\
& \quad=A e^{-\int^{u} \frac{B(u)}{A} d u} \partial_{u}\left[e^{+\int^{u} \frac{B(u)}{A} d u} \tilde{\phi}_{\left\{k_{+} l \vec{k} r\right\}}\right]=0,
\end{aligned}
$$

with

$$
\begin{aligned}
A & =\left(-2 i k_{+}\right), \\
B(u) & =\left(-\vec{k}^{2}-r\right)+\left(-i k_{+}\right) \frac{1}{u}+\frac{-l^{2}}{\Delta^{2}} \frac{1}{u^{2}},
\end{aligned}
$$

and this in turn implies the absence of the oscillating factor $e^{i \frac{\mathcal{A}}{u}}$ when $l$ vanishes. It follows that any deformation which makes the coefficient of the highest order singularity not vanishing will do the trick.

The first and easiest possibility is to add a Wilson line along $z$, i.e. $a=\theta d z$. This shifts $l \rightarrow l-e \theta$ and regularizes the scalar QED. Unfortunately this does not work for String Theory where Wilson lines on $D 25$ branes are not felt by the neutral strings starting and ending on the same brane. This happens because not all interactions involve commutators of the Chan-Paton factors which vanish for neutral strings. For instance the interaction among two tachyons and the first massive state involves an anti-commutator as we discuss later. The anti-commutators are present also in amplitudes of supersymmetric strings with massive states and therefore the issue is not solved by supersymmetry.

A second possibility is to think about higher derivative couplings to curvature which is also natural in String Theory 
If we regularize the metric in a minimal way as shown at the end of Sect. 2.1, we see that only $R i c_{u u}$ is non vanishing, therefore it would be natural to introduce

$$
\begin{aligned}
& S_{\text {higher R }}=\int_{\Omega} \mathrm{d}^{D} x \sqrt{-\operatorname{det} g} \\
& \quad \times\left(\sum_{k \geq 1} \alpha^{\prime 2 k-1} \prod_{j=1}^{k} g^{\mu_{j} v_{j}} g^{\rho_{j} \sigma_{j}} \operatorname{Ric}_{\mu_{j} \rho_{j}}\right. \\
& \left.\quad \times\left(\sum_{s=0}^{2 k} c_{k s} \partial^{2 k-s} \phi^{*} \partial^{s} \phi\right)\right) \\
& =\int_{\Omega} \mathrm{d}^{D} x \sqrt{-\operatorname{det} g}\left(\alpha^{\prime} g^{\mu \nu} g^{\rho \sigma} \operatorname{Ric}_{\mu \rho}\right. \\
& \left.\quad \times\left(c_{12} \phi^{*} \partial_{\nu \sigma}^{2} \phi+c_{11} \partial_{\nu} \phi^{*} \partial_{\sigma} \phi+c_{10} \partial_{\nu \sigma}^{2} \phi^{*} \phi\right)\right),
\end{aligned}
$$

where $\alpha^{\prime}$ has been introduced for dimensional reasons and in order to have all $c$ 's adimensional. Since only $R i c_{u u}$ is non vanishing and it depends only on $u$, the regularized d'Alembertian eigenmode problem would now read

$$
\begin{gathered}
-2 \partial_{u} \partial_{v} \phi_{r}-\frac{u}{u^{2}+\epsilon^{2}} \partial_{v} \phi_{r}+\frac{1}{\Delta^{2}\left(u^{2}+\epsilon^{2}\right)} \partial_{z}^{2} \phi_{r} \\
+\sum_{k \geq 1} \alpha^{\prime 2 k-1} C_{k} \operatorname{Ric}_{u u}^{k} \partial_{v}^{2 k} \phi+\partial_{i}^{2} \phi_{r}-r \phi_{r}=0
\end{gathered}
$$

with $C_{k}=\sum_{s=0}^{2 k}(-)^{s} c_{k s}$. We can perform the usual Fourier transform and the function $B(u)$ becomes

$$
\begin{aligned}
B(u)= & \left(-\vec{k}^{2}-r\right)+\left(-i k_{+}\right) \frac{u}{u^{2}+\epsilon^{2}}+\frac{-l^{2}}{\Delta^{2}} \frac{1}{u^{2}+\epsilon^{2}} \\
& +\sum_{k \geq 1} \alpha^{\prime 2 k-1} C_{k}\left(\frac{\epsilon^{2}}{\left(u^{2}+\epsilon^{2}\right)^{2}}\right)^{k}\left(-i k_{+}\right)^{2 k} .
\end{aligned}
$$

Then we examine what happens when $u=0$ :

$$
B(0) \sim \frac{-l^{2}}{\Delta^{2}} \frac{1}{\epsilon^{2}}+\sum_{k \geq 1} \alpha^{2 k-1} C_{k}\left(-i k_{+}\right)^{2 k} \frac{1}{\epsilon^{2 k}} .
$$

Even though it looks as if it presents the possibility to cure the issue, unfortunately it is not so. If we consider $\alpha^{\prime}$ and $\epsilon^{2}$ uncorrelated we lose predictability but if we consider $\alpha^{\prime} \sim$ $\epsilon^{2}$, as it is natural in String Theory, we do not solve the problem since $B(0) \sim \frac{-l^{2}}{\Delta^{2}} \frac{1}{\epsilon^{2}}+\sum_{k \geq 1} C_{k}\left(-i k_{+}\right)^{2 k} \epsilon^{2 k-2}$ and the curvature terms are not anymore singular.

\subsection{A hope from twisted state background}

It is clear from the previous discussion that the true problem is associated with the dipole string and its charge neutral states since the charged ones can be cured rather trivially by a Wilson line.
On the other side we know that the usual time-like orbifolds are well defined because of a presence of a $B_{\mu \nu}$ background and this field is sourced by strings. So we can think of switching on such a background in the open string. For open strings $B$ is equivalent to $F$ so we can consider what happens to an open string in an electromagnetic background.

The choice of such a background is limited first of all by the request that it must be an exact string solution, i.e. that it satisfies the e.o.m derived from the DBI. If a closed string winds the compact direction $z$ is coupled to $B_{z u}, B_{z v}$ and $B_{z i}$ but if we choose

$\frac{1}{2 \pi \alpha^{\prime}} B=f(u) d u \wedge d z$.

then

$\operatorname{det}\left(g+2 \pi \alpha^{\prime} f\right)=\operatorname{det}(g)$,

therefore it is a solution of open string e.o.m. for any $f\left(u, v, z, x^{i}\right)$. Suppose that the action for a real neutral scalar $\phi$ is given by (as the 2 tachyons -2 photons amplitude suggests)

$$
\begin{aligned}
& S_{\text {Scalar kin }}=\int_{\Omega} d^{D} x \sqrt{-\operatorname{det} g} \frac{1}{2} \\
& \quad \times\left(-g^{\alpha \beta} \partial_{\alpha} \phi \partial_{\beta} \phi-M^{2} \phi^{2}+c_{1} \alpha^{\prime 2} \partial_{\mu} \phi \partial_{\nu} \phi f^{\mu \kappa} f_{\kappa}^{v}\right) \\
& =\int d^{D-3} \vec{x} \int d u \int d v \int_{0}^{2 \pi} d z|\Delta u| \frac{1}{2} \\
& \quad \times\left(2 \partial_{u} \phi \partial_{v} \phi-\frac{1}{(\Delta u)^{2}}\left(\partial_{z} \phi\right)^{2}\right. \\
& \left.\quad-(\vec{\partial} \phi)^{2}-M^{2} \phi^{2}+c_{1} \alpha^{\prime 2} \frac{1}{(\Delta u)^{2}}\left(\partial_{v} \phi\right)^{2} f^{2}(u)\right),
\end{aligned}
$$

Performing the same steps as before we get

$$
\begin{aligned}
B(u)= & \left(-\vec{k}^{2}-r\right)+\left(-i k_{+}\right) \frac{1}{u} \\
& +\frac{\left(-l^{2}+c_{1} \alpha^{\prime 2} f(u)^{2} k_{+}{ }^{2}\right)}{\Delta^{2} u^{2}},
\end{aligned}
$$

so even for a constant $f(u)=f_{0}$ we get a solution which solves the issues. Notice however that the "trivial" solution $f=f_{0} d u \wedge d z$ is not so trivial in Minkowski coordinates $f=\frac{f_{0}}{x^{-}} d x^{-} \wedge d x^{2}$. Though appealing, the study of the string in the presence of this non trivial background needs a deeper analysis which goes beyond the scope of this paper.

\section{NBOeigenfunctions from covering space}

In this section we recover the eigenfunctions from the covering Minkowski space in order to elucidate the connection 
between the polarizations in NBOand in Minkowski. Moreover we want to generalize the result to a symmetric two index tensor which is the polarization of the first massive state to compute the two tachyons one massive state in the next section and to show that it diverges.

\subsection{Spin 0 wave function from Minkowski space}

We start with the usual plane wave in flat space and we express it in the new coordinates (we do not write the dependence on $\vec{x}$ since it is trivial)

$$
\begin{aligned}
& \psi_{k_{+} k_{-} k_{2}}\left(x^{+}, x^{-}, x^{2}\right) \\
& =e^{i\left(k_{+} x^{+}+k_{-} x^{-}+k_{2} x^{2}\right)}=\psi_{k_{+} k_{-} k_{2}}(u, v, z) \\
& =e^{i\left[k_{+} v+\frac{2 k_{+} k_{--k_{2}}}{2 k_{+}} u+\frac{1}{2} \Delta^{2} k_{+} u\left(z+\frac{k_{2}}{\Delta k_{+}}\right)^{2}\right]} .
\end{aligned}
$$

The corresponding wave function on the NBOis obtained by making it periodical in $z$. This can be done in two ways either in $x$ coordinates or in $u v z$ ones. The first way is more useful in deducing how the passage to the orbifold makes the function depend on the equivalence class of momenta. Implementing the projection on periodic $z$ functions we get

$$
\begin{aligned}
\Psi_{\left[k_{+} k_{-} k_{2}\right]}\left(\left[x^{+}, x^{-}, x^{2}\right]\right) & =\sum_{n \in \mathbb{Z}} \psi_{k_{+} k_{-} k_{2}}\left(\mathcal{K}^{n}\left(x^{+}, x^{-}, x^{2}\right)\right) \\
& =\sum_{n \in \mathbb{Z}} \psi_{\mathcal{K}^{-n}\left(k_{+} k_{-} k_{2}\right)}\left(x^{+}, x^{-}, x^{2}\right),
\end{aligned}
$$

where we write $\left[k_{+} k_{-} k_{2}\right]$ because the function depends on the equivalence class of $k_{+} k_{-} k_{2}$ only. The equivalence relation is given by

$$
\begin{aligned}
k & =\left(\begin{array}{l}
k_{+} \\
k_{-} \\
k_{2}
\end{array}\right) \equiv \mathcal{K}^{-n} k \\
& =\left(\begin{array}{c}
k_{+}+n(2 \pi \Delta) k_{2}+\frac{1}{2} n^{2}(2 \pi \Delta)^{2} k_{+} \\
k_{2}+n(2 \pi \Delta) k_{+}
\end{array}\right),
\end{aligned}
$$

and allows to choose a representative with

$$
\left\{\begin{array}{l}
0 \leq \frac{k_{2}}{\Delta \mid k_{+}}<2 \pi \quad k_{+} \neq 0 \\
0 \leq \frac{k_{-}}{\Delta\left|k_{2}\right|}<2 \pi \quad k_{+}=0, k_{2} \neq 0
\end{array} .\right.
$$

If we perform the computation in $u v z$ coordinates we get

$$
\begin{aligned}
& \Psi_{\left[k_{+} k_{-} k_{2}\right]}(u, v, z) \\
& \quad=\sum_{n \in \mathbb{Z}} \psi_{k_{+} k_{-} k_{2}}(u, v, z+2 \pi n) \\
& =\sum_{n \in \mathbb{Z}} e^{i\left\{k_{+} v+\frac{r}{2 k_{+}} u+\frac{1}{2}(2 \pi \Delta)^{2} k_{+} u\left[n+\frac{1}{2 \pi}\left(z+\frac{k_{2}}{\Delta k_{+}}\right)\right]^{2}\right\},}
\end{aligned}
$$

with $r=2 k_{+} k_{-}-k_{2}^{2}$ and $\operatorname{Im}\left(k_{+} u\right)>0$, i.e. $k_{+} u=$ $\left|k_{+} u\right| e^{i \epsilon}$ and $\pi>\epsilon>0$. Notice that there is no separate dependence on $z$ and on $\frac{k_{2}}{\Delta k_{+}}$therefore one could fix the range $0 \leq z+\frac{k_{2}}{\Delta k_{+}}<2 \pi$. However this symmetry is broken when considering the photon eigenfunction.

We can now use the Poisson resummation

$$
\begin{aligned}
\sum_{n} e^{i a(n+b)^{2}} & =\int \mathrm{d} s \delta_{P}(s) e^{i a(s+b)^{2}} \\
& =(2 \pi)^{2} \frac{\left.e^{-i\left(\frac{\pi}{4}+\frac{1}{2} \arg (a)\right.}\right)}{2 \sqrt{\pi|a|}} \sum_{m} e^{+\frac{\pi^{2} m^{2}}{i a}+i 2 \pi b m},
\end{aligned}
$$

to finally get, reintroducing the other variables $\vec{k}, \vec{x}$ and setting therefore $r=2 k_{+} k_{-}-k_{2}^{2}-\vec{k}^{2}$,

$$
\begin{aligned}
& \Psi_{\left[k_{+} k_{-} k_{2} \vec{k}\right]}(u, v, z, \vec{x}) \\
& =(2 \pi)^{2} \sqrt{\frac{2}{\pi}} \frac{e^{-i \pi / 4}(2 \pi \Delta)}{} \\
& \quad \times \sum_{l}\left[\frac{1}{\sqrt{\left|k_{+} u\right|}} e^{i\left\{k_{+} v+l z-\frac{l^{2}}{2 \Delta^{2} k_{+}} \frac{1}{u}+\frac{r+\vec{k}^{2}}{2 k_{+}} u+\vec{k} \cdot \vec{x}\right\}}\right] e^{i l \frac{k_{2}}{\Delta k_{+}}} \\
& =\mathcal{N} \sum_{l} \phi_{\left\{k_{+} l \vec{k} r\right\}}(u, v, z, \vec{x}) e^{i l \frac{k_{2}}{\Delta k_{+}}} \quad \text { when } k_{+} \neq 0,
\end{aligned}
$$

with

$\mathcal{N}=\sqrt{\frac{(2 \pi)^{D}}{\pi \Delta}} \frac{e^{-i \pi / 4}}{\pi}$.

The fact that $\Psi$ depends only on the equivalence class $\left[k_{+} k_{-} k_{2} \vec{k}\right]$ allows to restrict to $0 \leq \frac{k_{2}}{\Delta\left|k_{+}\right|}<2 \pi$ so that we can invert the previous expression and get

$$
\begin{aligned}
& \phi_{\left\{k_{+} l \vec{k} r\right\}}(u, v, z, \vec{x}) \\
& \quad=\frac{1}{\mathcal{N}} \int_{0}^{2 \pi \Delta\left|k_{+}\right|} \frac{\mathrm{d} k_{2}}{2 \pi \Delta\left|k_{+}\right|} e^{-i l \frac{k_{2}}{\Delta k_{+}}} \Psi_{\left[k_{+} k_{-} k_{2} \vec{k}\right]}(u, v, z, \vec{x}) .
\end{aligned}
$$

\subsection{Spin 1 wave function from Minkowski space}

We can repeat the steps of the previous section in the case of an electromagnetic wave. Again we concentrate on $x^{+}, x^{-}$ and $x^{2}$ coordinates and reinstate $\vec{x}$ at the end. We start with the usual plane wave in flat space $\psi_{k_{+} k_{-} k_{2}, \epsilon_{+} \epsilon_{-} \epsilon_{2}}$ and we express it in both Minkowskian and orbifold coordinates. We use the notation $\psi_{k_{+} k_{-} k_{2}, \epsilon_{+} \epsilon_{-} \epsilon_{2}}^{[0]}$ to stress that it is the eigenfunction and not the field which is obtained as

$A_{\mu}(x) d x^{\mu}=\int \mathrm{d}^{3} k \sum_{\epsilon} \psi_{k_{+} k_{-} k_{2}, \epsilon_{+} \epsilon_{-} \epsilon_{2}}^{[1]}$, 
where the sum is performed over $\epsilon$ which are independent and compatible with $k$. The explicit expression for the eigenfunction with $\epsilon$ constant is ${ }^{6}$

$$
\begin{aligned}
\mathcal{N} & \psi_{k_{+} k_{-} k_{2}, \epsilon_{+} \epsilon_{-} \epsilon_{2}}^{[1]}\left(x^{+}, x^{-}, x^{2}\right) \\
= & \left(\epsilon_{+} d x^{+}+\epsilon_{-} d x^{-}+\epsilon_{2} d x^{2}\right) e^{i\left(k_{+} x^{+}+k_{-} x^{-}+k_{2} x^{2}\right)} \\
= & \mathcal{N} \psi_{k_{+} k_{-} k_{2}, \epsilon_{+} \epsilon_{-} \epsilon_{2}}^{[1]}(u, v, z) \\
= & \left(\epsilon_{u} \mathrm{~d} u+\epsilon_{z} \mathrm{~d} z+\epsilon_{v} \mathrm{~d} v\right) \\
& e^{i\left[k_{+} v+\frac{2 k_{+} k_{-}-k_{2}}{2 k_{+}} u+\frac{1}{2} \Delta^{2} k_{+} u\left(z+\frac{k_{2}}{\Delta k_{+}}\right)^{2}\right],}
\end{aligned}
$$

with

$$
\begin{aligned}
\epsilon_{v} & =\epsilon_{+} \\
\epsilon_{u}(z) & =\epsilon_{-}+(\Delta z) \epsilon_{2}+\left(\frac{1}{2} \Delta^{2} z^{2}\right) \epsilon_{+}, \\
\epsilon_{z}(u, z) & =(\Delta u)\left(\epsilon_{2}+\Delta z \epsilon_{+}\right) .
\end{aligned}
$$

Notice that we are not yet imposing any gauge and also that if $\left(\epsilon_{+}, \epsilon_{-}, \epsilon_{2}\right)$ are constant $\left(\epsilon_{u}, \epsilon_{v}, \epsilon_{z}\right)$ are generically functions but it is worth stressing that $\left(\epsilon_{u}, \epsilon_{v}, \epsilon_{z}\right)$ are not the polarizations in the orbifold which are anyhow constant, the fact that they depend on the coordinates is simply the statement that not all eigenfunctions of the vector d'Alembertian are equal.

Building the corresponding function on the orbifold amounts to summing the images

$$
\begin{aligned}
\mathcal{N} \Psi_{[k, \epsilon]}^{[1]}([x]) & =\sum_{n} \epsilon \cdot\left(\mathcal{K}^{-n} \mathrm{~d} x\right) \psi_{k}\left(\mathcal{K}^{-n} x\right) \\
& =\sum_{n} \mathcal{K}^{n} \epsilon \cdot \mathrm{d} x \psi_{\mathcal{K}^{n} k}(x),
\end{aligned}
$$

this expression makes clear that under the action of the Killing vector $\epsilon$ transforms exactly as the $k$ since it is induced by $\epsilon \cdot \mathcal{K}^{n} \mathrm{~d} x=\mathcal{K}^{-n} \epsilon \cdot \mathrm{d} x$, i.e.

$$
\begin{aligned}
\epsilon & =\left(\begin{array}{c}
\epsilon_{+} \\
\epsilon_{2} \\
\epsilon_{-}
\end{array}\right) \equiv \mathcal{K}^{-n} \epsilon \\
& =\left(\begin{array}{c}
\epsilon_{+} \\
\epsilon_{2}+n(2 \pi \Delta) \epsilon_{+} \\
\epsilon_{-}+n(2 \pi \Delta) \epsilon_{2}+\frac{1}{2} n^{2}(2 \pi \Delta)^{2} \epsilon_{+}
\end{array}\right),
\end{aligned}
$$

however the pair $(k, \epsilon)$ transforms with the same $n$ since both are "dual" to $x$, i.e. their transformation rules are dictated by the $x$. Therefore there is only one equivalence class $[k, \epsilon]$ and

\footnotetext{
${ }^{6}$ We introduce the normalization factor $\mathcal{N}$ in order to have a less cluttered relation between $\epsilon$ and $\mathcal{E}$.
}

not two $[k],[\epsilon]$. Said differently, a representative of the combined equivalence class is the one with $0 \leq k_{2}<2 \pi \Delta\left|k_{+}\right|$ when $k_{+} \neq 0$.

We now proceed to find the eigenfunctions on the orbifold in orbifold coordinates. We notice that $\mathrm{d} u, \mathrm{~d} v$ and $\mathrm{d} z$ are invariant and therefore their coefficients in $a$ are as well. So we write

$$
\begin{aligned}
\mathcal{N} & \Psi_{[k, \epsilon]}^{[1]}([x]) \\
= & \sum_{n} \epsilon \cdot\left(\mathcal{K}^{n} \mathrm{~d} x\right) \psi_{k}\left(\mathcal{K}^{n} x\right) \\
= & \mathrm{d} v\left[\epsilon_{+} \sum_{n} \psi_{k}\left(\mathcal{K}^{n} x\right)\right]+\mathrm{d} z(\Delta u) \\
& {\left[\epsilon_{2} \sum_{n} \psi_{k}\left(\mathcal{K}^{n} x\right)+\epsilon_{+} \Delta \sum_{n}(z+2 \pi n) \psi_{k}\left(\mathcal{K}^{n} x\right)\right] } \\
& +\mathrm{d} u\left[\epsilon_{-} \sum_{n} \psi_{k}\left(\mathcal{K}^{n} x\right)+\epsilon_{2} \Delta \sum_{n}(z+2 \pi n) \psi_{k}\left(\mathcal{K}^{n} x\right)\right. \\
& \left.+\frac{1}{2} \epsilon_{+} \Delta^{2} \sum_{n}(z+2 \pi n)^{2} \psi_{k}\left(\mathcal{K}^{n} x\right)\right] .
\end{aligned}
$$

From direct computation we get $^{7}$

$$
\begin{aligned}
& \sum_{n}(z+2 \pi n) \psi_{k}\left(\mathcal{K}^{n} x\right)=\left(\frac{1}{i \Delta u} \frac{\partial}{\partial k_{2}}-\frac{k_{2}}{\Delta k_{+}}\right) \Psi_{[k]}([x]) \\
& \sum_{n}(z+2 \pi n)^{2} \psi_{k}\left(\mathcal{K}^{n} x\right)=\left(\frac{1}{i \Delta u} \frac{\partial}{\partial k_{2}}-\frac{k_{2}}{\Delta k_{+}}\right)^{2} \Psi_{[k]}([x]) .
\end{aligned}
$$

Then it follows that

$$
\begin{aligned}
& \mathcal{N} \Psi_{[k, \epsilon]}^{[1]}([x]) \\
& =\mathrm{d} v\left[\epsilon_{+} \Psi_{[k]}([x])\right]+\mathrm{d} z(\Delta u) \\
& {\left[\frac{\epsilon_{2} k_{+}-\epsilon_{+} k_{2}}{k_{+}} \Psi_{[k]}([x])+\epsilon_{+} \frac{-i}{u} \frac{\partial}{\partial k_{2}} \Psi_{[k]}([x])\right]} \\
& +\mathrm{d} u\left[\left(\epsilon_{-}-\epsilon_{2} \frac{k_{2}}{k_{+}}+\frac{1}{2} \epsilon_{+}\left(\frac{k_{2}}{k_{+}}\right)^{2}\right) \Psi_{[k]}([x])\right. \\
& +\frac{i}{2 u} \frac{\epsilon_{+}}{k_{+}} \Psi_{[k]}([x]) \\
& +\frac{\epsilon_{2} k_{+}-\epsilon_{+} k_{2}}{k_{+}} \frac{-i}{u} \frac{\partial}{\partial k_{2}} \Psi_{[k]}([x]) \\
& \left.+\frac{1}{2} \epsilon_{+} \frac{-1}{u^{2}} \frac{\partial^{2}}{\partial k_{2}^{2}} \Psi_{[k]}([x])\right]
\end{aligned}
$$

where many coefficients of $\Psi$ or its derivatives contain $k_{2}$. They cannot be expressed using the quantum numbers of

\footnotetext{
${ }_{7}$ Notice that these expressions may be written using Hermite polynomials.
} 
the orbifold $\left\{k_{+} l \vec{k} r\right\}$ but are invariant on the orbifold and therefore are new orbifold quantities which we can interpret as orbifold polarizations. Using (3.7) we can finally write

$$
\begin{aligned}
& \Psi_{[k, \epsilon]}^{[1]]}([x]) \\
& =\sum_{l} \phi_{\left\{k_{+} l \vec{k} r\right\}}(u, v, z, \vec{x}) e^{i l \frac{k_{2}}{\Delta k_{+}}} \\
& \quad \times\left\{\mathrm{d} v\left[\epsilon_{+}\right]+\mathrm{d} z(\Delta u)\left[\frac{\epsilon_{2} k_{+}-\epsilon_{+} k_{2}}{k_{+}}+\epsilon_{+} \frac{1}{\Delta u} \frac{l}{k_{+}}\right]\right. \\
& \quad+\mathrm{d} u\left[\left(\epsilon_{-}-\epsilon_{2} \frac{k_{2}}{k_{+}}+\frac{1}{2} \epsilon_{+}\left(\frac{k_{2}}{k_{+}}\right)^{2}\right)\right. \\
& \quad+\frac{i}{2 u} \frac{\epsilon_{+}}{k_{+}}+\frac{\epsilon_{2} k_{+}-\epsilon_{+} k_{2}}{k_{+}} \frac{1}{u} \frac{l}{\Delta k_{+}} \\
& \left.\left.\quad+\epsilon_{+} \frac{1}{2 u^{2}}\left(\frac{l}{\Delta k_{+}}\right)^{2}\right]\right\} .
\end{aligned}
$$

If we compare with (2.27) we find

$$
\begin{aligned}
& \mathcal{E}_{\left\{k_{+} l \vec{k} r\right\} \underline{v}}=\epsilon_{+} \\
& \mathcal{E}_{\left\{k_{+} l \vec{k} r\right\}_{\underline{z}}}=\operatorname{sgn}(u) \frac{\epsilon_{2} k_{+}-\epsilon_{+} k_{2}}{k_{+}} \\
& \mathcal{E}_{\left\{k_{+} l \vec{k} r\right\}_{\underline{u}}}=\epsilon_{-}-\epsilon_{2} \frac{k_{2}}{k_{+}}+\frac{1}{2} \epsilon_{+}\left(\frac{k_{2}}{k_{+}}\right)^{2},
\end{aligned}
$$

which implies that the true polarizations $\left(\epsilon_{+}, \epsilon_{-}, \epsilon_{2}\right)$ and $\mathcal{E}_{\left\{k_{+} l \vec{k} r\right\}_{*}}$ are constant as it turns out from direct computation.

A different way of reading the previous result is that the polarizations on the orbifold are the coefficients of the highest power of $u$.

We can also invert the previous relations to get

$$
\begin{aligned}
\epsilon_{+}= & \mathcal{E}_{\left\{k_{+} l \vec{k} r\right\} \underline{v}} \\
\epsilon_{2}= & \mathcal{E}_{\left\{k_{+} l \vec{k} r\right\}_{\underline{z}}} \operatorname{sgn}(u)+\frac{k_{2}}{k_{+}} \mathcal{E}_{\left\{k_{+} l \vec{k} r\right\} \underline{v}} \\
\epsilon_{-}= & \mathcal{E}_{\left\{k_{+} l \vec{k} r\right\}_{\underline{u}}}+\frac{k_{2}}{k_{+}} \mathcal{E}_{\left\{k_{+} l \vec{k} r\right\}_{\underline{z}}} \operatorname{sgn}(u) \\
& +\frac{1}{2}\left(\frac{k_{2}}{k_{+}}\right)^{2} \mathcal{E}_{\left\{k_{+} l \vec{k} r\right\} \underline{v}},
\end{aligned}
$$

and use them in Lorenz gauge $k \cdot \epsilon=0$ in order to get the expression of Lorenz gauge with orbifold polarizations. If the definition of orbifold polarizations is right the result cannot depend on $k_{2}$ since $k_{2}$ is not a quantum number of orbifold eigenfunctions. Taking in account $k_{-}=\frac{\vec{k}^{2}+k_{2}^{2}+r}{2 k_{+}}$ in $k \cdot \epsilon=0$ we get exactly the expression for the Lorenz gauge for orbifold polarizations (2.25).

\subsection{Tensor wave function from Minkowski space}

Once again, we can use the analysis of the previous section in the case of a second order symmetric tensor wave function. Again we suppress the dependence on $\vec{x}$ and $\vec{k}$ with a caveat: the Minkowskian polarizations $S_{+i}, S_{-i}$ and $S_{2 i}$ do transform non trivially, therefore we give the full expressions in Appendix A even if these components contribute in a somewhat trivial way since they behave effectively as a vector of the orbifold.

We start with the usual wave in flat space and we express either in the Minkowskian coordinates

$$
\begin{aligned}
\mathcal{N} \psi_{k S}^{[2]}\left(x^{+}, x^{-}, x^{2}\right)= & S_{\mu \nu} \psi_{k}(x) \mathrm{d} x^{\mu} \mathrm{d} x^{\nu} \\
= & \left(S_{++} \mathrm{d} x^{+} \mathrm{d} x^{+}+2 S_{+2} \mathrm{~d} x^{+} \mathrm{d} x^{2}\right. \\
& +2 S_{+-} \mathrm{d} x^{+} \mathrm{d} x^{-} \\
& +2 S_{22} \mathrm{~d} x^{2} \mathrm{~d} x^{2}+2 S_{2-} \mathrm{d} x^{2} \mathrm{~d} x^{-} \\
& \left.+2 S_{--} \mathrm{d} x^{-} \mathrm{d} x^{-}\right) e^{i\left(k_{+} x^{+}+k_{-} x^{-}+k_{2} x^{2}\right)}
\end{aligned}
$$

or in orbifold coordinates

$$
\begin{aligned}
& \mathcal{N} \psi_{k S}^{[2]}(x)=S_{\alpha \beta} \psi_{k}(x) \mathrm{d} x^{\alpha} \mathrm{d} x^{\beta} \\
&=\{(\mathrm{d} v)^{2}\left[S_{++}\right] \\
&+\mathrm{d} v \mathrm{~d} z \Delta u\left[2 S_{+2}+S_{++} \Delta z\right] \\
& \quad+\mathrm{d} v \mathrm{~d} u\left[2 S_{+-}+2 S_{+2} \Delta z+S_{++} \Delta^{2} z^{2}\right] \\
&+\mathrm{d} z^{2} \Delta^{2} u^{2}\left[S_{22}+2 S_{+2} \Delta z+S_{++} \Delta^{2} z^{2}\right] \\
&+\mathrm{d} z \mathrm{~d} v \Delta u \\
& \times\left[2 S_{-2}+2\left(S_{22}+S_{+-}\right) \Delta z+3 S_{+2} \Delta^{2} z^{2}+S_{++} \Delta^{3} z^{3}\right] \\
&+\mathrm{d} u^{2}\left[S_{--}+2 S_{-2} \Delta z+\left(S_{22}+S_{+-}\right) \Delta^{2} z^{2}\right. \\
&\left.\left.+S_{+2} \Delta^{3} z^{3}+\frac{1}{4} S_{++} \Delta^{4} z^{4}\right]\right\} \\
& \quad e^{i\left[k_{+} v+\frac{2 k_{+} k_{-} k_{2}}{2 k_{+}} u+\frac{1}{2} \Delta^{2} k_{+} u\left(z+\frac{k_{2}}{\Delta k_{+}}\right)^{2}\right] .}
\end{aligned}
$$

Now we define the tensor on the orbifold as a sum over all images as

$$
\begin{aligned}
\mathcal{N} \Psi_{[k S]}^{[2]}([x]) & =\sum_{n}\left(\mathcal{K}^{n} d x\right) \cdot S \cdot\left(\mathcal{K}^{n} \mathrm{~d} x\right) \psi_{k}\left(\mathcal{K}^{n} x\right) \\
& =\sum_{n} \mathrm{~d} x \cdot\left(\mathcal{K}^{-n} S\right) \cdot \mathrm{d} x \psi_{\mathcal{K}^{-n} k}(x) .
\end{aligned}
$$


In the last line we have defined the induced action of the Killing vector on $(k, S)$ which can be explicitly written as

$$
\begin{aligned}
\mathcal{K}^{-n}\left(\begin{array}{l}
S_{++} \\
S_{+2} \\
S_{+-} \\
S_{22} \\
S_{2-} \\
S_{--}
\end{array}\right) \\
=\left(\begin{array}{l}
S_{++} \\
S_{+2}+n \Delta S_{++} \\
S_{+-}+n \Delta S_{+2}+\frac{1}{2} n^{2} \Delta^{2} S_{++} \\
S_{22}+2 n \Delta S_{+2}+n^{2} \Delta^{2} S_{++} \\
S_{2-}+n \Delta\left(S_{22}+S_{+-}\right)+\frac{3}{2} n^{2} \Delta^{2} S_{+2}+\frac{1}{2} n^{3} \Delta^{3} S_{++} \\
S_{--}+2 n \Delta S_{-2}+n^{2} \Delta^{2}\left(S_{22}+S_{+-}\right)+n^{3} \Delta^{3} S_{+2}+\frac{1}{4} n^{4} \Delta^{4} S_{++}
\end{array}\right)
\end{aligned}
$$

In orbifold coordinates to compute the tensor on the orbifold simply amounts to sum over all the shifts $z \rightarrow(z+2 \pi n)$ and the use of the generalization of (3.16), i.e. to substitute $(\Delta z)^{j} \psi_{k} \rightarrow\left(\frac{1}{i u} \frac{\partial}{\partial k_{2}}-\frac{k_{2}}{\Delta k_{+}}\right)^{j} \Psi_{[k]}([x])$. When expressing all in the $\phi$ basis this last step is equivalent to $(\Delta z)^{j} \psi_{k} \rightarrow$ $\left(\frac{l}{\Delta u k_{+}}\right)^{j}+\ldots$ We identify the basic polaritazions on the orbifold by considering the highest power in $u$ and get

$$
\begin{aligned}
\mathcal{S}_{u u}= & \frac{1}{4} K^{4} S_{++}+K^{2} S_{+-}-K^{3} S_{+2} \\
& +S_{--}-2 K S_{-2}+S_{22} K^{2} \\
\mathcal{S}_{u v}= & \frac{1}{2} K^{2} S_{++}+S_{+-}-K S_{+2} \\
\mathcal{S}_{u z}= & -\frac{1}{2} K^{3} S_{++}-K S_{+-}+\frac{3}{2} K^{2} S_{+2}+S_{-2}-K S_{22} \\
\mathcal{S}_{v v}= & S_{++} \\
\mathcal{S}_{v z}= & S_{+2} K S_{++} \\
\mathcal{S}_{z z}= & K^{2} S_{++}-2 K S_{+2}+S_{22} .
\end{aligned}
$$

with $K=\frac{k_{2}}{k_{+}}$. The previous equations can be inverted into

$$
\begin{aligned}
S_{--}= & K^{2}\left(\mathcal{S}_{z z}+\mathcal{S}_{u v}\right)+K^{3} \mathcal{S}_{v z} \\
& +\frac{1}{4} K^{4} \mathcal{S}_{v v}+2 K \mathcal{S}_{u z}+\mathcal{S}_{u u} \\
S_{+-}= & K \mathcal{S}_{v z}+\frac{1}{2} K^{2} \mathcal{S}_{v v}+\mathcal{S}_{u v} \\
S_{-2}= & K\left(\mathcal{S}_{z z}+\mathcal{S}_{u v}\right)+\frac{3}{2} K^{2} \mathcal{S}_{v z}+\frac{1}{2} K^{3} \mathcal{S}_{v v}+\mathcal{S}_{u z} \\
S_{++}= & \mathcal{S}_{v v} \\
S_{+2}= & \mathcal{S}_{v z}+K \mathcal{S}_{v v} \\
S_{22}= & \mathcal{S}_{z z}+2 K \mathcal{S}_{v z}+K^{2} \mathcal{S}_{v v} .
\end{aligned}
$$

Since we plan to use the previous quantities in the case of the first massive string state we compute the relevant quantities: the trace

$\operatorname{tr}(S)=\mathcal{S}_{z z}-2 \mathcal{S}_{u v}$

and the transversality conditions

$$
\begin{aligned}
\operatorname{trans} \mathcal{S}_{v} & =(k \cdot S)_{+}=-\frac{\left(r+\vec{k}^{2}\right)}{2 k_{+}} \mathcal{S}_{v v}-k_{+} \mathcal{S}_{u v}, \\
\operatorname{trans} \mathcal{S}_{z} & =(k \cdot S)_{2}-K(k \cdot S)_{+} \\
& =-\frac{\left(r+\vec{k}^{2}\right)}{2 k_{+}} \mathcal{S}_{v z}-k_{+} \mathcal{S}_{u z}, \\
\operatorname{trans} \mathcal{S}_{u} & =(k \cdot S)_{-}-K(k \cdot S)_{2}+\frac{1}{2} K^{2}(k \cdot S)_{+} \\
& =-\frac{\left(r+\vec{k}^{2}\right)}{2 k_{+}} \mathcal{S}_{u v}-k_{+} \mathcal{S}_{u u} .
\end{aligned}
$$

where we used $k_{-}=\left(r+\vec{k}^{2}+k_{2}^{2}\right) /\left(2 k_{+}\right)$. These conditions correctly do no depend on $K$ since $k_{2}$ is not an orbifold quantum number.

The final expression for the orbifold symmetric tensor is

$$
\begin{aligned}
& \Psi_{[k, S]}^{[2]}([x])=\sum_{l} \phi_{\left\{k_{+} l \vec{k} r\right\}}(u, v, z, \vec{x}) e^{i l \frac{k_{2}}{\Delta k_{+}}} \\
& \times\left\{(\mathrm{d} v)^{2}\left[\mathcal{S}_{v v}\right]+2 \Delta u \mathrm{~d} v \mathrm{~d} z\left[\mathcal{S}_{v z}+\left(\frac{L \mathcal{S}_{v v}}{\Delta}\right) \frac{1}{u}\right]\right. \\
& +2 \mathrm{~d} v \mathrm{~d} u \\
& \times\left[\mathcal{S}_{u v}+\left(\frac{L \mathcal{S}_{v z}}{\Delta}+\frac{i \mathcal{S}_{v v}}{2 k_{+}}\right) \frac{1}{u}+\left(\frac{L^{2} \mathcal{S}_{v v}}{2 \Delta^{2}}\right) \frac{1}{u^{2}}\right] \\
& +(\Delta u)^{2} \mathrm{~d} z^{2} \\
& \times\left[\mathcal{S}_{z z}+\left(\frac{2 L \mathcal{S}_{v z}}{\Delta}+\frac{i \mathcal{S}_{v v}}{k_{+}}\right) \frac{1}{u}+\left(\frac{L^{2} \mathcal{S}_{v v}}{\Delta^{2}}\right) \frac{1}{u^{2}}\right] \\
& +2 \Delta u \mathrm{~d} z \mathrm{~d} u \\
& \times\left[\mathcal{S}_{u z}+\left(\frac{L \mathcal{S}_{z z}}{\Delta}+\frac{3 i \mathcal{S}_{v z}}{2 k_{+}}+\frac{L \mathcal{S}_{u v}}{\Delta}\right) \frac{1}{u}\right. \\
& \left.+\left(\frac{3 L^{2} \mathcal{S}_{v z}}{2 \Delta^{2}}+\frac{3 i L \mathcal{S}_{v v}}{2 \Delta k_{+}}\right) \frac{1}{u^{2}}+\left(\frac{L^{3} \mathcal{S}_{v v}}{2 \Delta^{3}}\right) \frac{1}{u^{3}}\right] \\
& +\mathrm{d} u^{2}\left[\mathcal{S}_{u u}+\left(\frac{i \mathcal{S}_{z z}}{k_{+}}+\frac{2 L \mathcal{S}_{u z}}{\Delta}+\frac{i \mathcal{S}_{u v}}{k_{+}}\right) \frac{1}{u}\right. \\
& +\left(\frac{L^{2} \mathcal{S}_{z z}}{\Delta^{2}}+\frac{3 i L \mathcal{S}_{v z}}{\Delta k_{+}}-\frac{3 \mathcal{S}_{v v}}{4 k_{+}^{2}}+\frac{L^{2} \mathcal{S}_{u v}}{\Delta^{2}}\right) \frac{1}{u^{2}} \\
& \left.\left.+\left(\frac{L^{3} \mathcal{S}_{v z}}{\Delta^{3}}+\frac{3 i L^{2} \mathcal{S}_{v v}}{2 \Delta^{2} k_{+}}\right) \frac{1}{u^{3}}+\left(\frac{L^{4} \mathcal{S}_{v v}}{4 \Delta^{4}}\right) \frac{1}{u^{4}}\right]\right\},
\end{aligned}
$$

with $L=\frac{l}{k_{+}}$. 


\section{Overlap of wave functions and their derivatives}

In this section we compute overlaps of wave functions and give their expressions both using integrals over the eigenfunctions and sum of products of $\delta$. The latter is the expression which is naturally obtained by computing tree level string amplitudes on the orbifold when one starts with Minkowski amplitudes and adds the amplitudes due to images. This is equivalent to compute emission vertices on the orbifold and then compute their correlations since this amounts to transferring the sum over the spacetime images to the sum of the polarizations images. We show this carefully in the next section. We consider also when and if they diverge. Finally we use the wording wave function for the functions on Minkowski space because we do not assume any constraint on polarizations.

\subsection{Overlaps without derivatives}

Let us start with the simplest case of the overlap of $N$ scalar wave function. We compute the overlap of orbifold wave functions and then we re-express it as sum of images of the corresponding Minkowski overlap thus establishing a dictionary between Minkowski and orbifold overlaps. Explicitly we consider the following overlap where all the polarizations $\mathcal{A}_{(i)}$ have been set to one

$$
\begin{aligned}
I^{(N)}= & \int_{\Omega} \mathrm{d}^{3} x \sqrt{-\operatorname{det} g} \\
& \left.\times \prod_{i=1}^{N} \Psi_{\left[k_{(i)+} k_{(i)-} k_{(i) 2}\right]}\left(\left[x^{+}, x^{-}, x^{2}\right]\right)\right) \\
= & \int_{\mathbb{R}^{1,2}} \mathrm{~d}^{3} x \sqrt{-\operatorname{det} g} \psi_{\left.k_{(1)+} k_{(1)-} k_{(1) 2}\left(x^{+}, x^{-}, x^{2}\right)\right)}^{N} \\
& \times \prod_{i=2} \sum_{m_{(i)} \in \mathbb{Z}} \psi_{k_{(i)+} k_{(i)-} k_{(i) 2}}\left(\mathcal{K}^{m_{(i)}}\left(x^{+}, x^{-}, x^{2}\right)\right) \\
= & \left.\int_{\mathbb{R}^{1,2}} \mathrm{~d}^{3} x \sqrt{-\operatorname{det} g} \psi_{k_{(1)+} k_{(1)-} k_{(1) 2}}\left(x^{+}, x^{-}, x^{2}\right)\right) \\
& \times \prod_{i=2}^{N} \sum_{m_{(i)} \in \mathbb{Z} \psi_{\mathcal{K}^{m}(i)}\left(k_{(i)+} k_{(i)-} k_{(i) 2}\right.}\left(x^{+}, x^{-}, x^{2}\right) \\
= & (2 \pi)^{3} \delta\left(\sum_{i} k_{(i)+}\right) \prod_{i=2}^{N} \sum_{m_{(i)} \in \mathbb{Z}} \delta \\
& \times\left.\left(\sum_{i} \mathcal{K}^{m_{(i)}} k_{(i) 2}\right) \delta\left(\sum_{i} \mathcal{K}^{\left.m_{(i)}\right)} k_{(i)-}\right)\right|_{m_{(1)}=0}
\end{aligned}
$$

where $\Omega=\mathbb{R}^{1,2} / \Gamma$ is the orbifold identified with the fundamental region of $\mathbb{R}^{1,2} / \Gamma$. We used the unfolding trick to rewrite the integral as an integral over $R^{1,2}$ thus dropping the sum over the images of particle (1). Then we moved the action of the Killing vector from $x$ to $k$ and finally we used the usual $\delta$ definition. The previous integral can be expressed explicitly as

$$
\begin{aligned}
I^{(N)}= & \mathcal{N}^{N} \sum_{\left\{l_{(i)}\right\} \in \mathbb{Z}^{N}} e^{i \sum_{i=1}^{N} l_{(i)} \frac{k_{(i) 2}}{\Delta k_{(i)+}}} \\
& \int_{\Omega} \mathrm{d}^{3} x \sqrt{-\operatorname{det} g} \prod_{i=1}^{N} \phi_{\left\{k_{(i)+} k_{(i)}-l_{(i)} r_{(i)}([x])\right)} \\
= & \mathcal{N}^{N} \sum_{\left\{l_{(i)\} \in \mathbb{Z}^{N}}\right.} e^{i \sum_{i=1}^{N} l_{(i)} \frac{k_{(i) 2}}{\Delta k_{(i)+}}}(2 \pi)^{2} \delta \\
& \left(\sum k_{(i)+}\right) \delta_{\sum l_{(i)}} \mathcal{I}_{\{N\}}^{[0]},
\end{aligned}
$$

from which we can reexpress the overlap of the wave functions using integrals over the infinite $\operatorname{sum} \delta^{2}$ as

$$
\begin{aligned}
\int_{\Omega} \mathrm{d}^{3} x \prod_{i=1}^{N} \phi_{\left\{k_{(i)+} k_{(i)-} l_{(i)} r_{(i)\}}([x])\right)} \\
=\frac{1}{\mathcal{N}^{N}} \prod_{i=1}^{N} \int_{0}^{2 \pi \Delta\left|k_{(i)+}\right|} \frac{\mathrm{d} k_{(i) 2}}{2 \pi \Delta\left|k_{(i)+}\right|} e^{-i l_{(i)} \frac{k_{(i) 2}}{\Delta k_{+}+} I^{(N)}} \\
=(2 \pi)^{3} \delta\left(\sum_{i} k_{(i)+}\right) \frac{1}{\mathcal{N}^{N}} \\
\times \prod_{i=1}^{N} \int_{0}^{2 \pi \Delta\left|k_{(i)+}\right|} \frac{\mathrm{d} k_{(i) 2}}{2 \pi \Delta\left|k_{(i)+}\right|} e^{-i l_{(i)} \frac{k_{(i) 2}}{\Delta k_{(i)+}}} \\
\quad \times \prod_{j=2}^{N} \sum_{m_{(j)} \in \mathbb{Z}} \delta\left(\sum_{j} \mathcal{K}^{m_{(j)}} k_{(j) 2}\right) \delta\left(\sum_{j} \mathcal{K}^{m_{(j)}} k_{(j)-}\right) .
\end{aligned}
$$

In particular it follows from the explicit expression of $\mathcal{I}_{\{n\}}^{[0]}$ that all overlaps $I^{(N)}$ for $N \geq 4$ are infinite.

Is there any intuitive reason for the divergence of the overlapping? We are summing over infinite distributions with accumulation points of their support. Nevertheless the existence of the accumulation point is not sufficient since the three scalars overlap, i.e. the three tachyons amplitude converges: the coefficients of the deltas matter, too, and the convergence must be verified.

\subsection{An overlap with one derivative}

Since we will also compute the amplitude involving two tachyons and one photon, as a preliminary step we consider the overlap in Minkowski space 


$$
\begin{aligned}
J_{\text {Mink }}= & i\left(\epsilon_{(1)} \cdot k_{(2) 2}\right)(2 \pi)^{3} \delta\left(\sum_{i} k_{(i)+}\right) \\
& \delta\left(\sum_{i} k_{(i) 2}\right) \delta\left(\sum_{i} k_{(i)-}\right) .
\end{aligned}
$$

Applying the recipe of summing over momentum and polarizations images of all but one particle, we can produce an expression which depends on equivalence classes as

$$
\begin{aligned}
& J\left(\left[k_{(1)}, \epsilon_{(1)}\right],\left[k_{(2)}\right],\left[k_{(3)}\right]\right)=i(2 \pi)^{3} \delta\left(\sum_{i} k_{(i)+}\right) \\
& \times \sum_{\left\{m_{(i)}\right\} \in \mathbb{Z}^{3}} \delta_{m_{(1)}, 1}\left(\mathcal{K}^{m_{(1)}} \epsilon_{(1)} \cdot \mathcal{K}^{m_{(2)}} k_{(2) 2}\right) \\
& \times \delta\left(\sum_{i} \mathcal{K}^{m_{(i)}} k_{(i) 2}\right) \delta\left(\sum_{i} \mathcal{K}^{m_{(i)}} k_{(i)-}\right) .
\end{aligned}
$$

This expression depends only on equivalence classes, for example under $\left(k_{(1)}, \epsilon_{(1)}\right) \rightarrow \mathcal{K}^{s}\left(k_{(1)}, \epsilon_{(1)}\right)$, we can use $\mathcal{K}^{s} a \cdot b=a \cdot \mathcal{K}^{-s} b$ and the invariance of deltas $\delta^{3}\left(\mathcal{K}^{s} a\right)=$ $\delta^{3}(a)$ to demonstrate it.

Now it is not difficult to show that the previous expression can be written as

$$
J=\int_{\Omega} \mathrm{d}^{3} x \eta^{\mu \nu} \Psi_{\left[k_{(1)}, \epsilon_{(1)}\right] \mu}^{[[]}([x]) \partial_{\nu} \Psi_{\left[k_{(2)}\right]}([x]) \Psi_{\left[k_{(3)}\right]}([x])
$$

where we performed the unfolding using $a_{\left[k_{(1)}, \epsilon_{(1)}\right] \mu}([x])$. Obviously we can perform the unfolding using whichever other field and this amount to keep the corresponding $m_{(i)}$ fixed in place of $m_{(1)}$.

Notice that the previous expression is invariant despite the fact that the derivatives $\partial_{\mu}$ are not well defined on the orbifold since not invariant and would hamper the use of the unfolding trick. The expression is invariant because $\Psi_{\mu}^{[1]}$ is not invariant too and compensates.

We can then evaluate the previous expression with Minkowskian polarizations using (3.18) which is nothing else but a rearrangement of terms of (4.5) to write

$$
\begin{aligned}
J= & i \mathcal{N}^{2} \sum_{\left\{l_{(i)}\right\} \in \mathbb{Z}^{3}} e^{i \sum_{i=1}^{3} l_{(i)} \frac{k_{(i) 2}}{\Delta k_{(i)+}}}(2 \pi)^{2} \delta\left(\sum k_{(i)+}\right) \delta_{\sum l_{(i)}} \\
& \times \int_{\Omega} \mathrm{d}^{3} x \prod_{i=1}^{3} \phi_{\left\{k_{(i)+} k_{(i)-} l_{(i)} r_{(i)}([x])\right)} \\
& \times\left\{\epsilon_{(1)+}\left[+\frac{i}{2 u}+\frac{l_{(2)}^{2}}{k_{(2)+}} \frac{1}{2 \Delta^{2} u^{2}}+\frac{r_{(2)}}{2 k_{(2)+}}\right]\right. \\
& +\frac{1}{\Delta u}\left[\epsilon_{(1) 2}+\frac{1}{\Delta u} \epsilon_{(1)+\frac{l_{(1)}}{k_{(1)+}}}\right] l_{(2)}
\end{aligned}
$$

$$
\begin{aligned}
& +\left[\epsilon_{(1)-}+\epsilon_{(1) 2} \frac{1}{\Delta u} \frac{l_{(1)}}{k_{(1)+}}+\epsilon_{(1)}+\frac{1}{2(\Delta u)^{2}} \frac{l_{(1)}{ }^{2}}{k_{(1)+}{ }^{2}}\right] \\
& \left.k_{(2)+}\right\} .
\end{aligned}
$$

Possible divergences come when $l=0$ because the absence of the factor $e^{i \frac{A}{u}}$, however all explicit factor $\frac{1}{u}$ come always with $l$ therefore when $l=0$ they do not give any contribution. A divergence when $l=0$ comes actually only from the contribution of the first line $\left.\partial_{u} \phi\right|_{l=0}=-\left.\frac{1}{2 u} \phi\right|_{l=0}$ but this cancels in scalar QED or with abelian tachyons because we have to subtract the contribution obtained exchanging (2) and (3). Because of color factors it does not cancel when considering the non abelian case unless one uses a kind of principal part prescription since replacing $\int_{-|a|}^{|b|} \mathrm{d} u \frac{\operatorname{sgn}(u)}{|u|^{3 / 2}}$ with $\lim _{\delta \rightarrow 0}\left[\int_{-|a|}^{-|\delta|}+\int_{-|\delta|}^{|b|}\right] \mathrm{d} u \frac{\operatorname{sgn}(u)}{|u|^{3 / 2}}$ gives a finite result.

\subsection{An overlap with two derivatives}

We can generalize the previous expressions to more general cases. Since we use the results from Sect. 3 we miss some non trivial contributions from polarizations like $\mathcal{S}_{v i}$. These contributions do not alter the discussion. However for completeness we give the lengthy full expression in Appendix B.

Having in mind the amplitudes with two tachyons and one massive state, we can consider an expression like

$$
\begin{aligned}
K= & \int_{\Omega} \mathrm{d}^{3} x \sqrt{-\operatorname{det} g} \eta^{\mu \nu} \eta^{\rho \sigma} \Psi_{\left[k_{(3)}, S_{(3)}\right] \mu \rho}^{[2]}([x]) \\
& \partial_{\nu \sigma}^{2} \Psi_{\left[k_{(2)}\right]}([x]) \Psi_{\left[k_{(1)}\right]}([x]),
\end{aligned}
$$

in Minkowskian coordinates or

$$
\begin{aligned}
K= & \int_{\Omega} \mathrm{d}^{3} x \sqrt{-\operatorname{det} g} g^{\alpha \beta} g^{\gamma \delta} \Psi_{\left[k_{(3)}, S_{(3)}\right] \alpha \gamma}^{[2]}([x]) D_{\beta} \\
& \partial_{\delta} \Psi_{\left[k_{(2)}\right]}([x]) \Psi_{\left[k_{(1)}\right]}([x])
\end{aligned}
$$

in orbifold coordinates where we need to use covariant derivatives. Using the unfolding trick over (3) we get

$$
\begin{aligned}
K= & (2 \pi)^{3} \delta\left(\sum_{i} k_{(i)+}\right) \\
& \times \prod_{i=2}^{N} \sum_{m_{(i)} \in \mathbb{Z}} S_{(3) \mu \rho}\left(\mathcal{K}^{m_{(2)}} k_{(2) 2}\right)^{\mu}\left(\mathcal{K}^{m_{(2)}} k_{(2) 2}\right)^{\rho} \\
& \times \delta\left(\sum_{i} \mathcal{K}^{m_{(i)}} k_{(i) 2}\right) \delta\left(\sum_{i} \mathcal{K}^{m_{(i)}} k_{(i)}\right)
\end{aligned}
$$

Explicitly in orbifold coordinates we can write 


$$
\begin{aligned}
K= & \int_{\Omega} \mathrm{d}^{3} x \sqrt{-\operatorname{det} g}\left[+\Psi_{\left[k_{(3)}, S_{(3)}\right] u u}^{[2]} \partial_{v}^{2} \Psi_{\left[k_{(2)}\right]}\right. \\
& -2 \frac{1}{(\Delta u)^{2}} \Psi_{\left[k_{(3)}, S_{(3)}\right] u z}^{[2]} \partial_{v} \partial_{z} \Psi_{\left[k_{(2)}\right]} \\
& +2 \Psi_{\left[k_{(3)}, S_{(3)}\right] u v}^{[2]} \partial_{v} \partial_{u} \Psi_{\left[k_{(2)}\right]} \\
& +\frac{1}{(\Delta u)^{4}} \Psi_{\left[k_{(3)}, S_{(3)}\right] z z}^{[2]}\left(\partial_{z}^{2} \Psi_{\left[k_{(2)}\right]}-\Delta^{2} u \partial_{v} \Psi_{\left[k_{(2)}\right]}\right) \\
& -2 \frac{1}{(\Delta u)^{2}} \Psi_{\left[k_{(3)}, S_{(3)}\right] z v}^{[2]}\left(\partial_{z} \partial_{u} \Psi_{\left[k_{(2)}\right]}-\frac{1}{u} \partial_{z} \Psi_{\left[k_{(2)}\right]}\right) \\
& \left.+\Psi_{\left[k_{(3)}, S_{(3)}\right] v v}^{[2]} \partial_{u}^{2} \Psi_{\left[k_{(2)}\right]}\right] \Psi_{\left[k_{(1)}\right]} .
\end{aligned}
$$

Keeping the terms which do not vanish when all $l=0$ and considering only the leading order in $\frac{1}{u}$ we get

$\left.K \sim \int \mathrm{d} u|u| \frac{3}{4} \frac{\left(k_{(2)+}+k_{(3)+}\right)^{2}}{k_{(3)+}{ }^{2}} \mathcal{S}_{v v(3)} \frac{1}{u^{2}} \prod_{i=1}^{3} \phi_{(i)}\right|_{l_{(*)}=0}$

which is divergent as $\frac{1}{|u|^{5 / 2}}$.

\section{String three points amplitudes with one massive state}

In this section we consider string amplitudes including string massive states. They are obtained using the inheritance principle and therefore they are connected to the integrals and relations derived in Sect. 4.

In particular we want to use the inheritance principle on the momenta and polarizations, i.e. we start form amplitudes in Minkowski expressed with momenta and polarizations and then we implement on them the projection to the orbifold. In particular it is worth stressing that as there is one Killing vector acting on the spacetime coordinates there is only one common Killing vector action on all the momenta and polarizations of each field as discussed in the spin-1 and spin2 cases. Moreover this approach gives the complete answer only tree level amplitudes since inside the loops twisted states may be created in pairs.

The final result is that the open string amplitude with two tachyons and the first massive (level 2) state diverges and there is no obvious way of curing it since the divergence is also present in the Abelian sector.

The open string expansion we use is

$$
\begin{aligned}
X(u, \bar{u})= & x_{0}-i 2 \alpha^{\prime} p \ln (|u|) \\
& +i \sqrt{\frac{\alpha^{\prime}}{2}} \sum_{n \neq 0} \frac{\alpha_{n}}{n}\left(u^{-n}+\bar{u}^{-n}\right) .
\end{aligned}
$$

\subsection{Level 2 massive state}

Before computing the amplitude we would like to review the possible polarizations of the first massive state in open string. The first massive vertex is

$$
\begin{aligned}
& V_{M}(x ; k, S, \xi)=:\left(\frac{i}{\sqrt{2 \alpha^{\prime}}} \xi \cdot \partial_{x}^{2} X(x, x)\right. \\
& \left.\quad+\left(\frac{i}{\sqrt{2 \alpha^{\prime}}}\right)^{2} S_{\mu \nu} \partial_{x} X^{\mu}(x, x) \partial_{x} X^{\nu}(x, x)\right) \\
& \quad \times e^{i k \cdot X(x, x)}:
\end{aligned}
$$

and the corresponding state is

$$
\begin{aligned}
& \lim _{x \rightarrow 0} V_{M}(x ; k, S, \xi)|0\rangle \\
& \quad=|k, S, \xi\rangle=\left(\xi \cdot \alpha_{-2}+\alpha_{-1} \cdot S \cdot \alpha_{-1}\right)|k\rangle .
\end{aligned}
$$

The physical conditions read

$$
\begin{aligned}
\left(L_{0}-1\right)|k, S, \xi\rangle & =0 \Rightarrow & \alpha^{\prime} k^{2}=-1 \\
L_{1}|k, S, \xi\rangle=0 \Rightarrow & & S \cdot k+\xi=0 \\
L_{2}|k, S, \xi\rangle=0 & \Rightarrow & k \cdot \xi+\operatorname{tr}(S)=0 .
\end{aligned}
$$

String gauge invariance allows to add

$L_{-1}\left(\chi \cdot \alpha_{-1}|k\rangle\right)=\left(\chi \cdot \alpha_{-2}+\chi \cdot \alpha_{-1} k \cdot \alpha_{-1}\right)|k\rangle$,

subject to the physical constraints, i.e.

$\alpha^{\prime} k^{2}=-1, \quad \chi \cdot k=0$.

Actually in critical string theory there is another gauge invariance generated by $L_{-2}+\frac{3}{2} L_{-1}^{2}$, in this case we can add a multiple of

$$
\begin{aligned}
& \left(L_{-2}+\frac{3}{2} L_{-1}^{2}\right)|k\rangle \\
& =\left(\frac{5}{2} k \cdot \alpha_{-2}+\frac{3}{2}\left(k \cdot \alpha_{-1}\right)^{2}+\frac{1}{2} \alpha_{-1}^{2}\right)|k\rangle
\end{aligned}
$$

to set $a=0$. Therefore the only non trivial d.o.f. are $S^{T T}$, i.e.

$\operatorname{tr}\left(S^{T T}\right)=k \cdot S^{T T}=\xi=0$.

In view of the computation for the orbifold we can check that given $k=\left(k_{+}, k_{-}, k_{2}, \vec{k}\right)\left(-2 k_{+} k_{-}+k_{2}^{2}+\vec{k}^{2}=-1\right)$ we can find a non trivial $S^{T T}$ with non vanishing components in the directions, \pm 2 only. We find in fact a two parameters family of solutions. The parameters may be taken to be $S_{++}$ 
and $S_{+2}$. Explicitly

$$
\begin{aligned}
&\left(\begin{array}{c}
S_{++} \\
S_{+-} \\
S_{+2} \\
S_{--} \\
S_{-2} \\
S_{22}
\end{array}\right)=\left(\begin{array}{c}
1 \\
-\frac{k_{-}}{k_{+}} \\
0 \\
\frac{k_{-}\left(k_{-} k_{+}-2 k_{2}^{2}\right)}{k_{+}^{3}} \\
-2 \frac{k_{-} k_{2}}{k_{+}{ }^{2}} \\
-2 \frac{k_{-}}{k_{+}}
\end{array}\right) S_{++} \\
& 0 \\
&+\left(\begin{array}{c}
\frac{k_{2}}{k_{+}} \\
1 \\
\frac{2 k_{2}\left(-k_{-} k_{+}+k_{2}{ }^{2}\right)}{k_{+}{ }^{3}} \\
\frac{k_{-} k_{+}-2 k_{2}^{2}}{k_{+}^{2}} \\
2 \frac{k_{2}}{k_{+}}
\end{array}\right) S_{+2}
\end{aligned}
$$

There is even a non trivial solution for the more special case $k=\left(k_{+}, k_{-}=1 / k_{+}, k_{2}=0, \overrightarrow{0}\right)$.

Similarly using the expressions for $S^{T T}$ in orbifold coordinates we check that there are two possible independent polarizations $\mathcal{S}_{v v}$ and $\mathcal{S}_{v z}$ which correspond to the the ones used above. Then the non trivial solution is

$$
\left(\begin{array}{c}
\mathcal{S}_{v v} \\
\mathcal{S}_{u v} \\
\mathcal{S}_{v z} \\
\mathcal{S}_{u u} \\
\mathcal{S}_{u z} \\
\mathcal{S}_{z z}
\end{array}\right)=\left(\begin{array}{c}
1 \\
-\frac{r+\vec{k}^{2}}{2 k_{+}{ }^{2}} \\
0 \\
\left(\frac{r+\vec{k}^{2}}{2 k_{+}{ }^{2}}\right)^{2} \\
0 \\
-2 \frac{r+\vec{k}^{2}}{2 k_{+}{ }^{2}}
\end{array}\right) \mathcal{S}_{v v}+\left(\begin{array}{c}
0 \\
-\frac{r+\vec{k}^{2}}{2 k_{+}{ }^{2}} \\
1 \\
0 \\
0 \\
0
\end{array}\right) \mathcal{S}_{v z .}
$$

\subsection{Two tachyons first massive state amplitude}

This Minkowskian full amplitude is given by the sum of two color ordered ones as

$$
\begin{aligned}
\mathcal{A}_{T T M}= & A_{T_{(1)} T_{(2)} M_{(3)}} \operatorname{tr}\left(T_{(1)} T_{(2)} T_{(3)}\right) \\
& +A_{T_{(2)} T_{(1)} M_{(3)}} \operatorname{tr}\left(T_{(2)} T_{(1)} T_{(3)}\right),
\end{aligned}
$$

where an easy computation gives

$$
\begin{aligned}
& A_{T_{(1)} T_{(2)} M_{3)}} \\
& \quad=\left\langle\left\langle k_{(1)}\right| V_{T}\left(1 ; k_{(2)}\right)\left(\alpha_{-1} \cdot S_{(3)}^{T T} \cdot \alpha_{-1}\left|k_{(3)}\right\rangle\right)\right. \\
& \quad=\left\langle\left\langle k_{(1)}\right| e^{i k_{(2)} \cdot x_{0}} e^{-\sqrt{2 \alpha^{\prime}} k_{(2)} \cdot \alpha_{1}}\left(\alpha_{-1} \cdot S_{(3)}^{T T} \cdot \alpha_{-1}\left|k_{(3)}\right\rangle\right)\right. \\
& =(2 \pi)^{D} \delta^{D}\left(\sum k_{(i)}\right)\left(\sqrt{2 \alpha^{\prime}}\right)^{2} k_{(2)} \cdot S_{(3)}^{T T} \cdot k_{(2)} \cdot
\end{aligned}
$$

Because of transversality of $S_{(3)}^{T T}$ the other term gives the same result of this one, hence the final Minkowskian amplitude is

$$
\begin{aligned}
\mathcal{A}_{T T M}= & (2 \pi)^{D} \delta^{D}\left(\sum k_{(i)}\right) 2\left(\sqrt{2 \alpha^{\prime}}\right)^{2} k_{(2)} \cdot S_{(3)}^{T T} \\
& \cdot k_{(2)} \operatorname{tr}\left(\left\{T_{(1)}, T_{(2)}\right\} T_{(3)}\right) .
\end{aligned}
$$

Then we can compute the orbifold amplitude as

$$
\begin{aligned}
& \mathcal{A}_{T T M}=(2 \pi)^{D-2} \delta^{D-3}\left(\sum \vec{k}_{(i)}\right) \delta\left(\sum k_{(i)+}\right) \\
& 2\left(\sqrt{2 \alpha^{\prime}}\right)^{2} \sum_{\left\{m_{(1)}, m_{(2)}, m_{(3)}\right\} \in \mathbb{Z}^{3}} \delta_{m_{(3)}, 1}\left(\mathcal{K}^{m_{(2)}} k_{(2)}\right) \cdot S_{(3)}^{T T} \\
& \\
& \cdot\left(\mathcal{K}^{m_{(2)}} k_{(2)}\right) \delta\left(\sum ( \mathcal { K } ^ { m _ { ( i ) } } k _ { ( i ) 2 } ) \delta \left(\sum\left(\mathcal{K}^{m_{(i)}} k_{(i)-}\right)\right.\right. \\
& \operatorname{tr}\left(\left\{T_{(1)}, T_{(2)}\right\} T_{(3)}\right) .
\end{aligned}
$$

The previous amplitude can then be expressed using an overlap as

$$
\begin{aligned}
\mathcal{A}_{T T M}= & 2\left(-i \sqrt{2 \alpha^{\prime}}\right)^{2} \int_{\Omega} \mathrm{d}^{3} x g^{\mu \nu} g^{\rho \sigma} \Psi_{\left[k_{(3)}, S_{(3)}\right] \mu \rho}^{[[]}([x]) \\
& \partial_{\nu \sigma}^{2} \Psi_{\left[k_{(2)}\right]}([x]) \Psi_{\left[k_{(1)}\right]}([x]) \operatorname{tr}\left(\left\{T_{(1)}, T_{(2)}\right\} T_{(3)}\right), \\
= & 2\left(-i \sqrt{2 \alpha^{\prime}}\right)^{2} \\
& \int_{\Omega} \mathrm{d}^{3} x g^{\alpha \beta} g^{\gamma \delta} \Psi_{\left[k_{(3)}, S_{(3)}\right] \alpha \gamma}^{[2]}([x]) D_{\beta} \\
& \partial_{\delta} \Psi_{\left[k_{(2)}\right]}([x]) \Psi_{\left[k_{(1)}\right]}([x]) \operatorname{tr}\left(\left\{T_{(1)}, T_{(2)}\right\} T_{(3)}\right) .
\end{aligned}
$$

As discussed in the Sect. 4 the last integral is divergent when $S_{++}=\mathcal{S}_{v v} \neq 0$ and the divergence cannot be avoided even introducing a Wilson line around $z$ since the amplitude involves an anticommutator which does not vanish in the Abelian sector.

\section{Scalar QED on GNBOand divergences}

As seen in the previous sections, the issues related to the vanishing volume of the compact directions lead to incurable divergences. We introduce the GNBOby inserting one additional non compact direction with respect to the NBOand show that divergences no longer occur.

As a parallel discussion to the NBO, we introduce the geometry of the GNBOand study scalar and spin-1 eigenfunctions to build the SQED on the orbifold. We then show how the presence of a non compact direction (we will stress the key differences from the NBO) can cure the theory when considering amplitudes and overlaps.

\subsection{Geometric preliminaries}

Consider Minkowski spacetime $\mathbb{R}^{1, D-1}$ and the change of coordinates from the lightcone set $\left(x^{\mu}\right)=\left(x^{+}, x^{-}, x^{2}, x^{3}, \vec{x}\right)$ 


$$
\begin{aligned}
& \text { to }\left(x^{\alpha}\right)=(u, v, w, z, \vec{x}) \text { : } \\
& \left\{\begin{array}{l}
x^{-}=u \\
x^{+}=v+\frac{\Delta_{2}^{2}}{2} u(z+w)^{2}+\frac{\Delta_{3}^{2}}{2} u(z-w)^{2} \\
x^{2}=\Delta_{2} u(z+w) \\
x^{3}=\Delta_{3} u(z-w)
\end{array}\right. \\
& \Leftrightarrow\left\{\begin{array}{l}
u=x^{-} \\
v=x^{+}-\frac{1}{2 x^{-}}\left(\left(x^{2}\right)^{2}+\left(x^{3}\right)^{2}\right) \\
w=\frac{1}{2 x^{-}}\left(\frac{x^{2}}{\Delta_{2}}-\frac{x^{3}}{\Delta_{3}}\right) \\
z=\frac{1}{2 x^{-}}\left(\frac{x^{2}}{\Delta_{2}}+\frac{x^{3}}{\Delta_{3}}\right)
\end{array}\right.
\end{aligned}
$$

where we do not perform any change on the transverse coordinates $\vec{x}$. The metric in these coordinates is non diagonal:

$$
\begin{aligned}
\mathrm{d} s^{2}= & -2 \mathrm{~d} u \mathrm{~d} v+\left(\Delta_{2}^{2}+\Delta_{3}^{2}\right) u^{2}\left(\mathrm{~d} w^{2}+\mathrm{d} z^{2}\right) \\
& +2\left(\Delta_{2}^{2}-\Delta_{3}^{2}\right) u^{2} \mathrm{~d} w \mathrm{~d} z+\eta_{i j} \mathrm{~d} x^{i} \mathrm{~d} x^{j}
\end{aligned}
$$

and its determinant is:

$$
-\operatorname{det} g=4 \Delta_{2}^{2} \Delta_{3}^{2} u^{4}
$$

From the previous expressions we can also derive the non vanishing Christoffel symbols:

$$
\begin{aligned}
& \Gamma_{w w}^{v}= \Gamma_{z z}^{v}=\left(\Delta_{2}^{2}+\Delta_{3}^{2}\right) u, \\
& \Gamma_{w z}^{v}=\left(\Delta_{2}^{2}-\Delta_{3}^{2}\right) u, \\
& \Gamma_{u w}^{w}=\Gamma_{u z}^{z}=\frac{1}{u},
\end{aligned}
$$

which however produce a vanishing Ricci tensor and curvature scalar, since we are considering Minkowski spacetime anyway.

We now introduce the GNBOby identifying points in space along the orbits of the Killing vector:

$$
\begin{aligned}
\kappa= & -2 \pi i\left(\Delta_{2} J_{+2}+\Delta_{3} J_{+3}\right) \\
= & 2 \pi\left(\Delta_{2} x^{2}+\Delta_{3} x^{3}\right) \partial_{+} \\
& +2 \pi \Delta_{2} x^{-} \partial_{2}+2 \pi \Delta_{3} x^{-} \partial_{3} \\
= & 2 \pi \partial_{z}
\end{aligned}
$$

in such a way that

$x^{\mu} \sim e^{n \kappa} x^{\mu}, \quad n \in \mathbb{Z}$

leads to the identifications

$$
x=\left(\begin{array}{c}
x^{-} \\
x^{2} \\
x^{3} \\
x^{+} \\
\vec{x}
\end{array}\right) \equiv \mathcal{K}^{n} x
$$

$$
=\left(\begin{array}{c}
x^{-} \\
x^{2}+2 \pi n \Delta_{2} x^{-} \\
x^{3}+2 \pi n \Delta_{3} x^{-} \\
x^{+}+2 \pi n \Delta_{2} x^{2}+2 \pi n \Delta_{3} x^{3}+(2 \pi n)^{2} \frac{\Delta_{2}^{2}+\Delta_{3}^{2}}{2} x^{-} \\
\vec{x}
\end{array}\right),
$$

or to the simpler

$(u, v, w, z) \sim(u, v, w, z+2 \pi n)$

using the map to the orbifold coordinates (6.1) where the Killing vector $\kappa=2 \pi \partial_{z}$ does not depend on the local spacetime configuration. As in the previous case, the difference between Minkowski spacetime and the GNBOis therefore global.

The geodesic distance between the nth copy and the base point on the orbifold can be computed in any set of coordinates and is:

$\Delta s_{(n)}^{2}=\left(\Delta_{2}^{2}+\Delta_{3}^{2}\right)\left(2 \pi n x^{-}\right)^{2} \geq 0$

Closed time-like curves are therefore avoided on the GNBQ, but there are closed null curves on the surface $x^{-}=u=0$ where the Killing vector $\kappa$ vanishes.

\subsection{Free scalar field}

In order to build a quantum theory on the GNBOusing Feynman's approach to quantization, we first solve the eigenvalue equations for the fields and then build their off-shell expansion. We start from a complex scalar field and then consider the free photon before moving to the sQED interactions on the GNBO

Consider the action for a complex scalar field:

$$
\begin{aligned}
& \mathrm{S}_{\text {Scalar kin }} \\
& =\int_{\Omega} \mathrm{d}^{D} x \sqrt{-\operatorname{det} g}\left(-g^{\mu \nu} \partial_{\mu} \phi^{*} \partial_{\nu} \phi-M^{2} \phi^{*} \phi\right) \\
& =\int_{\mathbb{R}^{D-4}} \mathrm{~d}^{D-4} \vec{x} \int_{-\infty}^{+\infty} \mathrm{d} u \int_{-\infty}^{+\infty} \mathrm{d} v \int_{-\infty}^{+\infty} \\
& \mathrm{d} w \int_{0}^{2 \pi} \mathrm{d} z 2\left|\Delta_{2} \Delta_{3}\right| u^{2} \\
& \quad \times\left[\partial_{u} \phi^{*} \partial_{v} \phi+\partial_{v} \phi^{*} \partial_{u} \phi\right. \\
& \quad-\frac{1}{4 u^{2}}\left(\left(\frac{1}{\Delta_{2}^{2}}+\frac{1}{\Delta_{3}^{2}}\right)\left(\partial_{w} \phi^{*} \partial_{w} \phi+\partial_{z} \phi^{*} \partial_{z} \phi\right)\right. \\
& \left.\quad+\left(\frac{1}{\Delta_{2}^{2}}-\frac{1}{\Delta_{3}^{2}}\right)\left(\partial_{w} \phi^{*} \partial_{z} \phi+\partial_{z} \phi^{*} \partial_{w} \phi\right)\right) \\
& \left.\quad-\eta^{i j} \partial_{i} \phi^{*} \partial_{j} \phi-M^{2} \phi^{*} \phi\right] .
\end{aligned}
$$


As in the case of the NBQ the solutions to the equations of motion are necessary to provide the modes of the quantum fields. We study the eigenvalue equation $\square \phi_{r}=r \phi_{r}$, where $r$ is $2 k_{+} k_{-}-\vec{k}$ by comparison with the flat case ( $k$ is the momentum associated to the flat coordinates). We therefore need solve:

$$
\begin{gathered}
\left\{-2 \partial_{u} \partial_{v}-\frac{2}{u} \partial_{v}+\frac{1}{4 u^{2}}\left[\left(\frac{1}{\Delta_{2}^{2}}+\frac{1}{\Delta_{3}^{2}}\right)\left(\partial_{w}^{2}+\partial_{z}^{2}\right)\right.\right. \\
\left.\left.+2\left(\frac{1}{\Delta_{2}^{2}}-\frac{1}{\Delta_{3}^{2}}\right) \partial_{w} \partial_{z}\right]+\eta^{i j} \partial_{i} \partial_{j}-r\right\} \phi_{r}=0
\end{gathered}
$$

To this purpose, we introduce a Fourier transformation over $v, w, z, \vec{x}$ :

$$
\begin{gathered}
\phi_{r}(u, v, w, z, \vec{x})=\sum_{l \in \mathbb{Z}} \int_{\mathbb{R}^{D-4}} \mathrm{~d}^{D-4} \vec{k} \int_{-\infty}^{+\infty} \mathrm{d} k_{+} \\
\int_{-\infty}^{+\infty} \mathrm{d} p e^{i\left(k_{+} v+p w+l z+\vec{k} \cdot \vec{x}\right)} \tilde{\phi}_{\left\{k_{+} p l \vec{k} r\right\}}(u),
\end{gathered}
$$

where we defined $k_{+}, p, l, \vec{k}$ as associated momenta to $v, w, z, \vec{x}$ respectively, and we find:

$$
\begin{aligned}
& \phi_{\left\{k_{+} p l \vec{k} r\right\}}(u, v, w, z, \vec{x}) \\
& \quad=e^{i\left(k_{+} v+p w+l z+\vec{k} \cdot \vec{x}\right)} \tilde{\phi}_{\left\{k_{+} p l \vec{k} r\right\}}(u) .
\end{aligned}
$$

where

$$
\begin{aligned}
\tilde{\phi}_{\left\{k_{+} p l \vec{k} r\right\}}(u)= & \frac{1}{2 \sqrt{(2 \pi)^{D}\left|\Delta_{2} \Delta_{3} k_{+}\right|}} \frac{1}{|u|} \\
& \times e^{-i\left(\frac{1}{8 k_{+} u}\left[\frac{(l+p)^{2}}{\Delta_{2}^{2}}+\frac{(l-p)^{2}}{\Delta_{3}^{2}}\right]-\frac{\vec{k}^{2}+r}{2 k_{+}} u\right) .}
\end{aligned}
$$

These solutions present the right normalization, as we can verify through the product:

$$
\begin{aligned}
& \left(\phi_{\left\{k_{(1)}+p_{(1)} l_{(1)} \vec{k}_{(1)} r_{(1)}\right\}}, \phi_{\left\{k_{(2)+} p_{(2)} l_{(2)} \vec{k}_{(2)} r_{(2)}\right\}}\right)=2\left|\Delta_{2} \Delta_{3}\right| \\
& \quad \times \int_{\mathbb{R}^{D-4}} \mathrm{~d}^{D-4} \vec{x} \int_{\mathbb{R}^{3}} \mathrm{~d} u \mathrm{~d} v \mathrm{~d} w \\
& \quad \int_{0}^{2 \pi} \mathrm{d} z u^{2} \phi_{\left\{k_{(1)+} p_{(1)} l_{(1)} \vec{k}_{(1)} r_{(1)}\right.} \phi_{\left\{k_{(2)+} p_{(2)} l_{(2)} \vec{k}_{(2)} r_{(2)}\right\}} \\
& =\delta^{D-4}\left(\vec{k}_{(1)}+\vec{k}_{(2)}\right) \delta\left(k_{(1)+}+k_{(2)+}\right) \delta\left(p_{(1)}+p_{(2)}\right) \\
& \quad \times \delta\left(r_{(1)}+r_{(2)}\right) \delta_{l_{(1)}, l_{(2)}} .
\end{aligned}
$$

Then we have the off-shell expansion:

$$
\begin{aligned}
\phi_{r}(u, v, w, z, \vec{x}) \\
=\frac{1}{2 \sqrt{(2 \pi)^{D}\left|\Delta_{2} \Delta_{3} k_{+}\right|}} \\
\quad \times \sum_{l \in \mathbb{Z}} \int_{\mathbb{R}^{D-4}} \mathrm{~d}^{D-4} \vec{k} \int_{-\infty}^{+\infty} \mathrm{d} k_{+} \int_{-\infty}^{+\infty} \mathrm{d} p \int_{-\infty}^{+\infty} \mathrm{d} r \\
\quad \times \frac{\mathcal{A}_{\left\{k_{+} p l \vec{k} r\right\}}}{|u|} \\
\quad \times e^{i\left(k+v+p w+l z+\vec{k} \cdot \vec{x}-\frac{1}{8 k_{+} u}\left[\frac{(l+p)^{2}}{\Delta_{2}^{2}}+\frac{(l-p)^{2}}{\Delta_{3}^{2}}\right]+\frac{\vec{k}^{2}+r}{2 k_{+}} u\right) .}
\end{aligned}
$$

\subsection{Free photon action}

We then study the action of the free photon field $a$ using the Lorenz gauge which in the orbifold coordinates it reads:

$$
\begin{aligned}
D^{\alpha} a_{\alpha}= & -\frac{2}{u} a_{v}-\partial_{v} a_{u}-\partial_{u} a_{v} \\
& +\frac{1}{4 u^{2}}\left(\left(\frac{1}{\Delta_{2}^{2}}+\frac{1}{\Delta_{3}^{2}}\right)\left(\partial_{w} a_{w}+\partial_{z} a_{z}\right)\right. \\
& \left.+\left(\frac{1}{\Delta_{2}^{2}}-\frac{1}{\Delta_{3}^{2}}\right)\left(\partial_{w} a_{z}+\partial_{z} a_{w}\right)\right) \\
& +\eta^{i j} \partial_{i} a_{j}=0 .
\end{aligned}
$$

We then solve the eigenvalue equations $\left(\square a_{r}\right)_{v}=r a_{r v}$, which in components read:

$$
\begin{aligned}
\left(\square a_{r}\right)_{u}= & \frac{2}{u^{2}} a_{r v}-\frac{1}{2 u^{3}} \\
& \times\left[\left(\frac{1}{\Delta_{2}^{2}}+\frac{1}{\Delta_{3}^{2}}\right)\left(\partial_{w} a_{r}+\partial_{z} a_{r z}\right)\right. \\
& \left.+\left(\frac{1}{\Delta_{2}^{2}}-\frac{1}{\Delta_{3}^{2}}\right)\left(\partial_{w} a_{r z}+\partial_{z} a_{r} w\right)\right] \\
& +\left\{-2 \partial_{u} \partial_{v}-\frac{2}{u} \partial_{v}+\frac{1}{4 u^{2}}\right. \\
& \times\left[\left(\frac{1}{\Delta_{2}^{2}}+\frac{1}{\Delta_{3}^{2}}\right)\left(\partial_{w}^{2}+\partial_{z}^{2}\right)+\left(\frac{1}{\Delta_{2}^{2}}-\frac{1}{\Delta_{3}^{2}}\right) 2 \partial_{w} \partial_{z}\right] \\
& \left.+\nabla_{T}^{2}\right\} a_{r u}, \\
\left(\square a_{r}\right)_{v}= & \left\{-2 \partial_{u} \partial_{v}-\frac{2}{u} \partial_{v}+\frac{1}{4 u^{2}}\right. \\
& {\left[\left(\frac{1}{\Delta_{2}^{2}}+\frac{1}{\Delta_{3}^{2}}\right)\left(\partial_{w}^{2}+\partial_{z}^{2}\right)+\left(\frac{1}{\Delta_{2}^{2}}-\frac{1}{\Delta_{3}^{2}}\right) 2 \partial_{w} \partial_{z}\right] } \\
& \left.+\nabla_{T}^{2}\right\} a_{r v}, \\
& -\frac{2}{u} \partial_{w} a_{r v}+\left\{-2 \partial_{u} \partial_{v}+\frac{1}{4 u^{2}}\right. \\
\left(\square a_{r}\right)_{w} & {\left[\left(\frac{1}{\Delta_{2}^{2}}+\frac{1}{\Delta_{3}^{2}}\right)\left(\partial_{w}^{2}+\partial_{z}^{2}\right)+\left(\frac{1}{\Delta_{2}^{2}}-\frac{1}{\Delta_{3}^{2}}\right) 2 a_{r} \partial_{w} \partial_{z}\right] } \\
&
\end{aligned}
$$




$$
\begin{aligned}
(\square a)_{z}= & -\frac{2}{u} \partial_{z} a_{r v}+\left\{-2 \partial_{u} \partial_{v}+\frac{1}{4 u^{2}}\right. \\
& {\left[\left(\frac{1}{\Delta_{2}^{2}}+\frac{1}{\Delta_{3}^{2}}\right)\left(\partial_{w}^{2}+\partial_{z}^{2}\right)+\left(\frac{1}{\Delta_{2}^{2}}-\frac{1}{\Delta_{3}^{2}}\right) 2 \partial_{w} \partial_{z}\right] } \\
& \left.+\nabla_{T}^{2}\right\} a_{r z}, \\
(\square a)_{i}= & \left\{-2 \partial_{u} \partial_{v}-\frac{2}{u} \partial_{v}+\frac{1}{4 u^{2}}\right. \\
& {\left[\left(\frac{1}{\Delta_{2}^{2}}+\frac{1}{\Delta_{3}^{2}}\right)\left(\partial_{w}^{2}+\partial_{z}^{2}\right)+\left(\frac{1}{\Delta_{2}^{2}}-\frac{1}{\Delta_{3}^{2}}\right) 2 \partial_{w} \partial_{z}\right] } \\
& \left.+\nabla_{T}^{2}\right\} a_{r i},
\end{aligned}
$$

where $\nabla_{T}^{2}=\eta^{i j} \partial_{i} \partial_{j}$ is the Laplace operator in the transverse coordinates $\vec{x}$. These equations can be solved using standard techniques through a Fourier transform:

$$
\begin{aligned}
& a_{r \alpha}(u, v, w, z, \vec{x}) \\
& =\sum_{l \in \mathbb{Z}} \int_{\mathbb{R}^{D-4}} \mathrm{~d}^{D-4} \vec{k} \int_{-\infty}^{+\infty} \mathrm{d} k_{+} \int_{-\infty}^{+\infty} \mathrm{d} p \\
& \left.\quad \times e^{i\left(k_{+} v+p w+l z+\vec{k} \cdot \vec{x}\right.}\right)_{\tilde{a}_{\left\{k_{+} p l \vec{k} r\right\} \alpha}(u) .}
\end{aligned}
$$

We first solve the equations for $\tilde{a}_{\left\{k_{+} p l \vec{k} r\right\} v}$ and $\tilde{a}_{\left\{k_{+} p l \vec{k} r\right\} i}$ since they are identical to the scalar equation (6.11). We then insert their solutions as sources for the equations for $\tilde{a}_{\left\{k_{+} p l \vec{k} r\right\} u}, \tilde{a}_{\left\{k_{+} p l \vec{k} r\right\} w}$ and $\tilde{a}_{\left\{k_{+} p l \vec{k} r\right\} z}$. The solutions can be written as the expansion:

$$
\begin{aligned}
& \left\|\tilde{a}_{\left\{k_{+} p l \vec{k} r\right\} \alpha}(u)\right\|=\left(\begin{array}{c}
\tilde{a}_{u} \\
\tilde{a}_{v} \\
\tilde{a}_{w} \\
\tilde{a}_{z} \\
\tilde{a}_{i}
\end{array}\right) \\
& =\sum_{\underline{\alpha} \in\{\underline{u}, \underline{v}, \underline{w}, \underline{z}, \underline{i}\}} \mathcal{E}_{\left\{k_{+} p l \vec{k} r\right\} \underline{\alpha}}\left\|\tilde{a}_{\left\{k_{+} p l \vec{k} r\right\} \alpha}(u)\right\|
\end{aligned}
$$

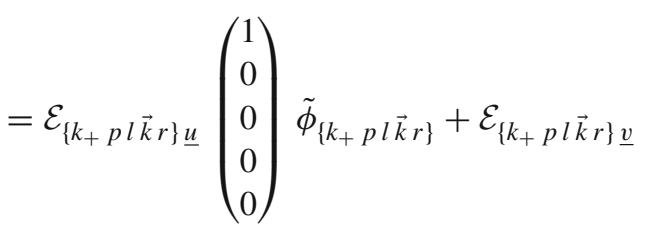

$$
\begin{aligned}
& \left(\begin{array}{c}
\frac{i}{2 k_{+} u}+\frac{1}{8 k_{+}{ }^{2} u^{2}}\left(\frac{(l+p)^{2}}{\Delta_{2}^{2}}+\frac{(l-p)^{2}}{\Delta_{3}^{2}}\right) \\
1 \\
\frac{p}{k_{+}} \\
\frac{l}{k_{+}} \\
0
\end{array}\right) \tilde{\phi}_{\left\{k_{+} p l \vec{k} r\right\}}
\end{aligned}
$$

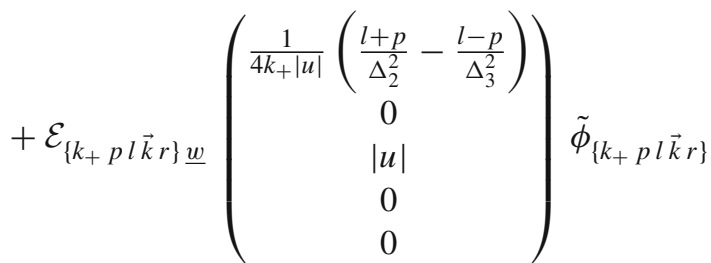

$$
\begin{aligned}
& +\mathcal{E}_{\left\{k_{+} p l \vec{k} r\right\} \underline{z}}\left(\begin{array}{c}
\frac{1}{4 k_{+}|u|}\left(\frac{l+p}{\Delta_{2}^{2}}+\frac{l-p}{\Delta_{3}^{2}}\right) \\
0 \\
0 \\
|u| \\
0
\end{array}\right) \tilde{\phi}_{\left\{k_{+} p l \vec{k} r\right\}}
\end{aligned}
$$

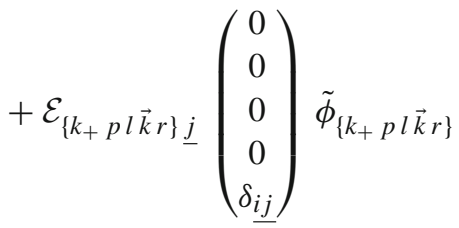

Consider the Fourier transformed functions:

$$
\begin{aligned}
& a_{\left\{k_{+} p l \vec{k} r\right\} \alpha}^{\alpha}(u, v, w, z, \vec{x}) \\
& \quad=e^{i\left(k_{+} v+p w+l z+\vec{k} \cdot \vec{x}\right)} \tilde{a}_{\left\{k_{+} p l \vec{k} r\right\} \alpha}^{\underline{\alpha}}(u),
\end{aligned}
$$

then we can expand the off shell fields as

$$
\begin{aligned}
a_{\alpha}(x)= & \sum_{l \in \mathbb{Z}} \int \mathrm{d}^{D-4} \vec{k} \int \mathrm{d} k_{+} \int \mathrm{d} p \int \mathrm{d} r \\
& \sum_{\underline{\alpha} \in\{\underline{u}, \underline{w}, \underline{w}, \underline{z}, \underline{i}\}} \mathcal{E}_{\left\{k_{+} l \vec{k} r\right\} \underline{\alpha}} a_{\left\{k_{+} p l \vec{k} r\right\} \alpha}(x) .
\end{aligned}
$$

We can compute the normalization as:

$$
\begin{aligned}
\left(a_{(1)}, a_{(2)}\right) & \\
= & \int_{\mathbb{R}^{D-4}} \mathrm{~d}^{D-4} \vec{x} \int_{\mathbb{R}^{3}} \mathrm{~d} u \mathrm{~d} v \mathrm{~d} w \int_{0}^{2 \pi} \mathrm{d} z, 2\left|\Delta_{2} \Delta_{3}\right| u^{2} \\
& \times\left(g^{\alpha \beta} a_{\left\{k_{(1)+} p_{(1)} l_{(1)} \vec{k}_{(1)} r_{(1)}\right\}} a_{\left\{k_{(2)+} p_{(2)} l_{(2)} \vec{k}_{(2)} r_{(2)}\right\}}\right) \\
= & \delta^{D-4}\left(\vec{k}_{(1)}+\vec{k}_{(2)}\right) \delta\left(p_{(1)}+p_{(2)}\right) \\
& \delta\left(k_{(1)+}+k_{(2)+}\right) \delta_{l_{(1)}+l_{(2)}, 0} \delta\left(r_{1}-r_{2}\right) \\
& \times \mathcal{E}_{\left\{k_{(1)+}\right.} p_{(1)} l_{(1)} \vec{k}_{(1)} r_{(1)} \circ \mathcal{E}_{\left\{k_{(2)+} p_{(2)} l_{(2)} \vec{k}_{(2)} r_{(2)}\right\}}
\end{aligned}
$$

where

$$
\begin{aligned}
\mathcal{E}_{(1)} \circ & \mathcal{E}_{(2)} \\
= & -\mathcal{E}_{(1) \underline{u}} \mathcal{E}_{(2) \underline{v}}-\mathcal{E}_{(1) \underline{v}} \mathcal{E}_{(2) \underline{u}} \\
+ & \frac{1}{4}\left[\left(\frac{1}{\Delta_{2}^{2}}+\frac{1}{\Delta_{3}^{2}}\right)\left(\mathcal{E}_{(1) \underline{w}} \mathcal{E}_{(2) \underline{w}}+\mathcal{E}_{(1) \underline{z}} \mathcal{E}_{(2) \underline{z}}\right)\right. \\
& \left.+\left(\frac{1}{\Delta_{2}^{2}}-\frac{1}{\Delta_{3}^{2}}\right)\left(\mathcal{E}_{(1) \underline{w}} \mathcal{E}_{(2) \underline{z}}+\mathcal{E}_{(1) \underline{z}} \mathcal{E}_{(2) \underline{w}}\right)\right]
\end{aligned}
$$

is independent of the coordinates. The Lorenz gauge now reads: 


$$
\begin{aligned}
& \eta^{i \underline{j}} k_{i} \mathcal{E}_{\left\{k_{+} p l \vec{k} r\right\} \underline{j}}-k_{+} \mathcal{E}_{\left\{k_{+} p l \vec{k} r\right\} \underline{u}} \\
& -\frac{\vec{k}^{2}+r}{2 k_{+}} \mathcal{E}_{\left\{k_{+} p l \vec{k} r\right\} \underline{v}}=0 .
\end{aligned}
$$

As in the previous case, it does not pose any constraint on the transverse polarizations $\mathcal{E}_{\left\{k_{+} p l \vec{k} r\right\} \underline{w}}$ and $\mathcal{E}_{\left\{k_{+} p l \vec{k} r\right\} \underline{z}}$.

\subsection{Cubic interaction}

As previously studied on the NBQ we can now show the SQED 3-points vertex computation using the previously computed eigenmodes. The presence of a continuous momentum in the non compact direction plays a major role in saving the convergence of the integrals. In the case of the GNBOwe find:

$$
\begin{aligned}
& \mathrm{S}_{\text {cubic }}=\int_{\Omega} \mathrm{d}^{D} x \sqrt{-\operatorname{det} g}\left(-i e g^{\mu v} a_{\mu}\left(\phi^{*} \partial_{\nu} \phi-\partial_{\nu} \phi^{*} \phi\right)\right) \\
& =\prod_{i=1}^{3} \sum_{l_{(i)} \in \mathbb{Z}} \int \mathrm{d}^{D-4} \vec{k}_{(i)} \int \mathrm{d} k_{(i)+} \int \mathrm{d} p_{(i)} \int \mathrm{d} r_{(i)} \\
& \times(2 \pi)^{D-1} \delta^{D-4}\left(\sum_{i=1}^{3} \vec{k}_{(i)}\right) \delta\left(\sum_{i=1}^{3} p_{(i)}\right) \\
& \delta\left(\sum_{i=1}^{3} k_{(i)+}\right) \delta_{\sum_{i=1}^{3} l_{(i)}, 0} \\
& \times e \mathcal{A}_{\left\{-k_{(2)+}-k w N 2-l_{(2)}-\vec{k}_{(2)} r_{(2)}\right\}} \mathcal{A}_{\left\{k_{(3)+} p_{(3)} l_{(3)} \vec{k}_{(3)} r_{(3)}\right\}} \\
& \times\left\{\mathcal{E}_{\left\{k_{(1)+} p_{(1)} l_{(1)} \vec{k}_{(1)} r_{(1)}\right\}} k_{(2)+} \mathcal{I}_{\{3\}}^{[0]}\right. \\
& \left.+\mathcal{E}_{\left\{k_{(1)+} p_{(1)}\right.} l_{(1)} \vec{k}_{(1)} r_{(1)}\right\} \underline{v} \\
& {\left[\left(\frac{\vec{k}_{(2)}^{2}+r_{(2)}}{2 k_{(2)+}}\right) \mathcal{I}_{\{3\}}^{[0]}+i \frac{k_{(2)+}}{k_{(1)+}} \mathcal{I}_{\{3\}}^{[-1]}+\frac{k_{(2)+}}{8}\right.} \\
& {\left[\frac{1}{\Delta_{2}^{2}}\left(\frac{l_{(1)}+p_{(1)}}{k_{(1)+}}+\frac{l_{(2)}+p_{(2)}}{k_{(2)+}}\right)^{2}\right.} \\
& \left.\left.+\frac{1}{\Delta_{3}^{2}}\left(\frac{l_{(1)}-p_{(1)}}{k_{(1)+}}+\frac{l_{(2)}-p_{(2)}}{k_{(2)+}}\right)^{2}\right] \mathcal{I}_{\{3\}}^{[-2]}\right] \\
& +\left(\mathcal{E}_{\left\{k_{(1)+} p_{(1)} l_{(1)} \vec{k}_{(1)} r_{(1)} \underline{w}\right.}-\mathcal{E}_{\left\{k_{(1)+} p_{(1)} l_{(1)} \vec{k}_{(1)} r_{(1)} \underline{z}\right.}\right) \\
& \times\left[\frac{1}{\Delta_{2}^{2}}\left(\frac{k_{(1)+}\left(l_{(2)}+p_{(2)}\right)+k_{(2)+}\left(l_{(1)}+p_{(1)}\right)}{k_{(1)+}}\right)\right. \\
& \left.-\frac{1}{\Delta_{3}^{2}}\left(\frac{k_{(1)+}\left(l_{(2)}-p_{(2)}\right)+k_{(2)+}\left(l_{(1)}-p_{(1)}\right)}{k_{(1)+}}\right)\right] \\
& \left.\mathcal{J}_{(3)}^{[-1]}+((2) \leftrightarrow(3))\right\}
\end{aligned}
$$

where we defined:

$\mathcal{I}_{\{N\}}^{[v]}=\int_{\mathbb{R}} \mathrm{d} u 2\left|\Delta_{2} \Delta_{3}\right| u^{2} u^{\nu} \prod_{i=1}^{N} \tilde{\phi}_{\left\{k_{(i)+} p_{(i)} l_{(i)} \vec{k}_{(i)} r_{(i)}\right\}}$,

$\mathcal{J}_{(N)}^{[v]}=\int_{\mathbb{R}} \mathrm{d} u 2\left|\Delta_{2} \Delta_{3}\right| u^{2}|u|^{v} \prod_{i=1}^{N} \tilde{\phi}_{\left\{k_{(i)+} p_{(i)} l_{(i)} \vec{k}_{(i)} r_{(i)}\right\}}$.

While in the NBOcase we need to regularize the integrals at least taking their principal part when all $l_{(*)}=0$ in (2.35), the GNBOdoes not need any specific manipulation. In fact the form of $\tilde{\phi}_{\left\{k_{(i)}+p_{(i)} l_{(i)} \vec{k}_{(i)} r_{(i)\}}\right.}$ in (6.14) prevents the formation of isolated zeros in the phase factor proportional to $u^{-1}$ : the presence of the continuous momentum $p$, contrary to the NBOwhere all momenta are discrete, gives the integrals a distributional interpretation, similar to a derivative of a Dirac $\delta$ function.

\subsection{Quartic interactions}

As for the NBO, we consider the quartic interaction for the sQED action:

$$
\begin{aligned}
& \mathrm{S}_{\text {quartic }}=\int_{\Omega} \mathrm{d}^{D} x \sqrt{-\operatorname{det} g}\left(e^{2} g^{\mu \nu} a_{\mu} a_{\nu}|\phi|^{2}-\frac{g_{4}}{4}|\phi|^{4}\right) \\
& \times \prod_{i=1}^{3}\left(\frac{1}{4 \pi \sqrt{\left((2 \pi)^{D} \mid \Delta_{2} \Delta_{3} k_{(i)+1}\right.}}\right) \\
& \times \sum_{l_{(i)} \in \mathbb{Z}} \int \mathrm{d}^{D-4} \vec{k}_{(i)} \int \mathrm{d} k_{(i)+} \int \mathrm{d} p_{(i)} \int \mathrm{d} r_{(i)} \\
& \times(2 \pi)^{D-1} \delta^{D-4}\left(\sum_{i=1}^{3} \vec{k}_{(i)}\right) \delta\left(\sum_{i=1}^{3} p_{(i)}\right) \\
& \delta\left(\sum_{i=1}^{3} k_{(i)+}\right) \delta_{\sum_{i=1}^{3} l_{(i)}, 0} \\
& \times\left\{e^{2} \mathcal{A}_{\left\{-k_{(3)+}-k w N 3-l_{(3)}-\vec{k}_{(3)} r_{(3)}\right\}} \mathcal{A}_{\left\{k_{(4)+} p_{(4)} l_{(4)} \vec{k}_{(4)} r_{(4)}\right\}}\right. \\
& \times\left[\mathcal{E}_{\left\{k_{(1)+} p_{(1)} l_{(1)} \vec{k}_{(1)} r_{(1)}\right\}} \circ \mathcal{E}_{\left\{k_{(2)+} p_{(2)} l_{(2)} \vec{k}_{(2)} r_{(2)}\right\}} \mathcal{I}_{\{4\}}^{[0]}\right.
\end{aligned}
$$

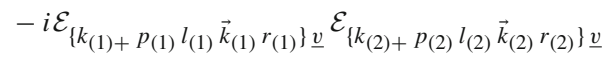

$$
\begin{aligned}
& \left(\left(\frac{1}{k_{(1)+}}+\frac{1}{k_{(2)+}}\right) \mathcal{I}_{\{4\}}^{[-1]}\right. \\
& \left.-i\left(\frac{\mathcal{G}_{+(1,2)}}{\Delta_{2}^{2}}+\frac{\mathcal{G}_{-(1,2)}}{\Delta_{3}^{2}}\right) \mathcal{I}_{\{4\}}^{[-2]}\right) \\
& \left.+\frac{1}{4}\left(\tilde{\mathcal{E}}_{+(1,2)} \frac{\mathcal{G}_{+(1,2)}}{\Delta_{2}^{2}}-\tilde{\mathcal{E}}_{-(1,2)} \frac{\mathcal{G}_{-(1,2)}}{\Delta_{2}^{2}}\right) \mathcal{J}_{(4)}^{[-1]}\right] \\
& -\frac{g_{4}}{4} \mathcal{A}_{\left\{-k_{(1)+}-k w N 1-l_{(1)}-\vec{k}_{(1)} r_{(1)}\right\}}
\end{aligned}
$$




$$
\begin{aligned}
& \mathcal{A}_{\left\{-k_{(2)+}-k w N 2-l_{(2)}-\vec{k}_{(2)} r_{(2)}\right\}}^{*} \\
& \left.\times \mathcal{A}_{\left\{k_{(3)+} p_{(3)} l_{(3)} \vec{k}_{(3)} r_{(3)}\right\}} \mathcal{A}_{\left\{k_{(4)+} p_{(4)} l_{(4)} \vec{k}_{(4)} r_{(4)}\right\}} \mathcal{I}_{\{4\}}^{[0]}\right\},
\end{aligned}
$$

where we defined:

$$
\begin{aligned}
\mathcal{G}_{ \pm(a, b)}= & \frac{l_{(a)} \pm p_{(a)}}{k_{(a)+}}-\frac{l_{(b)} \pm p_{(b)}}{k_{(b)+}}, \\
\tilde{\mathcal{E}}_{ \pm(a, b)}= & \mathcal{E}_{\left\{k_{(a)+} p_{(a)} l_{(a)} \vec{k}_{(a)} r_{(a)}\right\} \underline{v}} \\
& \times\left(\mathcal{E}_{\left\{k_{(b)+} p_{(b)} l_{(b)} \vec{k}_{(b)} r_{(b)}\right\} \underline{w}} \pm \mathcal{E}_{\left\{k_{(b)+} p_{(b)} l_{(b)} \vec{k}_{(b)} r_{(b)}\right\}}\right) \\
& -\mathcal{E}_{\left\{k_{(b)+} p_{(b)} l_{(b)} \vec{k}_{(b)} r_{(b)}\right\} \underline{v}} \\
& \times\left(\mathcal{E}_{\left\{k_{(a)+} p_{(a)} l_{(a)} \vec{k}_{(a)} r_{(a)}\right\} \underline{w}} \pm \mathcal{E}_{\left\{k_{(a)+} p_{(a)} l_{(a)} \vec{k}_{(a)} r_{(a)}\right\} \underline{z}}\right)
\end{aligned}
$$

for simplicity.

As the four points function in the NBOcase shows with clear evidence the presence of divergences when all $l_{(*)}=0$, the GNBOallows a distributional interpretation of the integrals $\mathcal{I}_{\{N\}}^{[\nu]}$ and $\mathcal{J}_{(N)}^{[\nu]}$ in the previous expression. In fact the regularization occurs in the same way as in the three points function in the GNBO the phase factor proportional to $u^{-1}$ has a continuous value due to the continuous momentum $p$ and it does not present isolated zeros which would prevent the interpretation as distribution.

\subsection{Resurgence of divergences}

Looking back at the metric (6.2) and at the identifications (6.8) where we compactified only the coordinate $z$ through the Killing vector $2 \pi \partial_{z}$ it seems reasonable to wonder what would happen if we acted in the same way over $w$, since $2 \pi \partial_{w}$ is a Killing vector as well and it commutes with $2 \pi \partial_{z}$. However the lesson we learnt from our whole study on NBOand GNBOis that in absence of at least one continuous transverse direction it is not possible to avoid the divergences associated with discrete zero energy modes and this is exactly what happens.

\subsection{A comment on the null brane regularization}

As mentioned in the introductory section, there have been attempts to regularize the NBOusing the Null Brane. Differently from the NBO, in this case the orbifold generator (2.8) includes an additional translation along an extra spatial dimension, namely

$$
\begin{aligned}
\kappa & =-2 \pi i \Delta J_{+2}-2 \pi i R P_{3} \\
& =2 \pi\left(\Delta \partial_{z}+R \partial_{3}\right) .
\end{aligned}
$$

with metric

$$
\mathrm{d} s^{2}=-2 \mathrm{~d} u \mathrm{~d} v+\Delta^{2} u^{2}(\mathrm{~d} z)^{2}+\left(\mathrm{d} x^{3}\right)^{2}+\eta_{i j} \mathrm{~d} x^{i} \mathrm{~d} x^{j}
$$

Even though similar in appearance to the GNBOKilling vector, this Killing vector is substantially different from (6.5).

The scalar field satisfies the same equation of motion as in the $\mathrm{NBO}$

$$
\begin{aligned}
& -2 \partial_{u} \partial_{v} \phi_{r}-\frac{1}{u} \partial_{v} \phi_{r}+\frac{1}{(u \Delta)^{2}} \partial_{z}^{2} \phi_{r}+\partial_{x^{3}}^{2} \phi_{r}+\eta^{i j} \partial_{i} \partial_{j} \phi_{r} \\
& =r \phi_{r}
\end{aligned}
$$

where $i, j=4,5, \ldots D-1$. The solution is as usual

$\tilde{\phi}_{\left\{k_{+} k_{z} k_{3} \vec{k} r\right\}}(u) \propto \frac{1}{\sqrt{|u|}} e^{-i \frac{k_{z}^{2}}{2 k_{+}} \frac{1}{u}+i \frac{k_{3}^{2}+\vec{k}^{2}+r}{2 k_{+}} u}$.

but with different periodicity conditions:

$e^{i 2 \pi n\left(\Delta k_{z}+R k_{3}\right)}=1$

This obscures the issue of the presence of a non compact direction. To show the non compact direction hidden in this system we may define the coordinates $\hat{z}=\frac{1}{2}\left(\frac{x^{3}}{R}+\frac{z}{\Delta}\right)$ and $\hat{x}^{3}=\frac{1}{2}\left(\frac{x^{3}}{R}-\frac{z}{\Delta}\right)$ such that $\kappa=2 \pi \partial_{\hat{z}}$ and

$$
\left(\begin{array}{c}
\hat{z} \\
\hat{x}^{3}
\end{array}\right) \equiv\left(\begin{array}{c}
\hat{z}+2 \pi n \\
\hat{x}^{3}
\end{array}\right)
$$

upon the orbifold identification. Then the momenta are $\hat{k}_{\hat{z}}=$ $\hat{l} \in \mathbb{Z}$ and $\hat{k}_{3} \in \mathbb{R}$ and they are related to the the momenta of the other coordinates as

$k_{3}=\frac{\hat{l}+\hat{k}_{3}}{2 R}, \quad k_{z}=\frac{\hat{l}-\hat{k}_{3}}{2 \Delta}$,

so that the solution can be written as

$\tilde{\phi}_{\left\{k_{+} \hat{l} \hat{k}_{3} \vec{k} r\right\}}(u) \propto \frac{1}{\sqrt{|u|}} e^{-i \frac{\left(\hat{l}-\hat{k}_{3}\right)^{2}}{8 \Delta^{2} k_{+}} \frac{1}{u}+i \frac{(2 R)^{-2}\left(\hat{k}_{3}-\hat{l}\right)^{2}+\vec{k}^{2}+r}{2 k_{+}} u}$,

which shows in a clear way that there is a non compact direction which allows a distributional interpretation as discussed in [3]. However this direction cannot be easily decoupled from the compact one.

\section{Quick analysis of the BO}

In this section we would like to quickly show the analysis performed in the previous sections for the NBObut in the case of the BO The results are not very different apart from the fact that divergences are milder, in fact it is possible to construct the full sQED but nevertheless it is impossible to consider higher derivative terms in the effective theory and some three point amplitudes with a massive state diverge. 


\subsection{Geometric preliminaries}

In $\mathbb{R}^{1,1}$ we consider the change of coordinates:

$\left\{\begin{array}{l}x^{+}=t e^{+\Delta \varphi} \\ x^{-}=\sigma_{-} t e^{-\Delta \varphi}\end{array} \Leftrightarrow\left\{\begin{array}{l}t=\operatorname{sgn}\left(x^{+}\right) \sqrt{\left|x^{+} x^{-}\right|} \\ \varphi=\frac{1}{2 \Delta} \log \left|\frac{x^{+}}{x^{-}}\right| \\ \sigma_{-}=\operatorname{sgn}\left(x^{+} x^{-}\right)\end{array}\right.\right.$

where $\sigma_{-}= \pm 1$ and $t, \varphi \in \mathbb{R}$. The metric reads

$$
\begin{aligned}
\mathrm{d} s^{2} & =-2 \mathrm{~d} x^{+} \mathrm{d} x^{+} \\
& =-2 \sigma_{-}\left(\mathrm{d} t^{2}-(\Delta t)^{2} \mathrm{~d} \varphi^{2}\right),
\end{aligned}
$$

and its determinant is:

$$
-\operatorname{det} g=4 \Delta^{2} t^{2} \text {. }
$$

In orbifold coordinates the non vanishing Christoffel symbols are:

$$
\Gamma_{\varphi \varphi}^{t}=\Delta^{2} t, \quad \Gamma_{t \varphi}^{\varphi}=\frac{1}{t} .
$$

Using the orbifold coordinates $(t, \varphi)$, the BOis obtained by requiring the identification $\varphi \equiv \varphi+2 \pi$ along the orbit of the global Killing vector $\kappa_{\varphi}=2 \pi \partial_{\varphi}$. We will therefore use the recurrent parameter $\Lambda=e^{2 \pi \Delta}$ in what follows.

\subsection{Free scalar action}

The action for a complex scalar $\phi$ is given by

$$
\begin{gathered}
S_{\text {Scalar kin }}=\int d^{D} x \sqrt{-\operatorname{det} g}\left(-g^{\mu \nu} \partial_{\mu} \phi^{*} \partial_{\nu} \phi-M^{2} \phi^{*} \phi\right) \\
=\sum_{\sigma_{-} \in\{ \pm 1\}} \int d^{D-2} \vec{x} \int_{-\infty}^{+\infty} d t \int_{0}^{2 \pi} d \phi \Delta|t| \\
\left(\frac{1}{2} \sigma_{-} \partial_{t} \phi^{*} \partial_{t} \phi+\frac{1}{2} \sigma_{-} \frac{1}{(\Delta t)^{2}} \partial_{\varphi} \phi^{*} \partial_{\varphi} \phi\right. \\
\left.-\partial_{i} \phi^{*} \partial_{i} \phi-M^{2} \phi^{*} \phi\right),
\end{gathered}
$$

As before we solve the associated eigenfunction problem for the d'Alembertian operator

$$
-\frac{1}{2} \sigma_{-} \partial_{t}^{2} \phi_{r}-\frac{1}{2} \sigma_{-} \frac{1}{t} \partial_{t} \phi_{r}+\frac{1}{2} \sigma_{-} \frac{1}{(\Delta t)^{2}} \partial_{\varphi}^{2} \phi_{r}+\partial_{i}^{2} \phi_{r}=r \phi_{r}
$$

with

$r=2 k_{+} k_{-}-\vec{k}^{2}=2 \varsigma_{-} m^{2}-\vec{k}^{2}$

where for later convenience (see the transformation of $k$ under the induced action of the Killing vector (7.17)) we parameterize the momenta as the coordinates

$$
\left\{\begin{array} { l } 
{ k _ { + } = m e ^ { + \Delta \beta } } \\
{ k _ { - } = \varsigma _ { - } m e ^ { - \Delta \beta } }
\end{array} \Leftrightarrow \left\{\begin{array}{l}
m=\operatorname{sgn}\left(k_{+}\right) \sqrt{\left|k_{+} k_{-}\right|} \\
\beta=\frac{1}{2 \Delta} \log \left|\frac{k_{+}}{k_{-}}\right| \\
\varsigma_{-}=\operatorname{sgn}\left(k_{+} k_{-}\right)
\end{array}\right.\right.
$$

where $\varsigma_{-}= \pm 1$ and $m, \beta \in \mathbb{R}$. To solve the problem we use standard techniques and perform the Fourier transform wrt $\vec{x}$ and $\phi$ as

$\phi(t, \varphi, \vec{x})=\int d^{D-2} \vec{x} \sum_{l \in \mathbb{Z}} e^{i \vec{k} \cdot \vec{x}} e^{i l \varphi} H_{l \vec{k} r \sigma_{-}}(t)$,

so that the new function $H_{l \vec{k} r \sigma_{-}}$satisfies

$$
\begin{aligned}
& \partial_{t}^{2} H_{l \vec{k} r \sigma_{-}}+\frac{1}{t} \partial_{t} H_{l \vec{k} r \sigma_{-}} \\
& \quad+\left[\frac{l^{2}}{(\Delta t)^{2}}+2 \sigma_{-}\left(r+\vec{k}^{2}\right)\right] H_{l \vec{k} r \sigma_{-}}=0,
\end{aligned}
$$

which upon the introduction of the natural quantities (see also (7.19) for an explanation of the naturalness of $\lambda$ )

$\tau=m t, \quad \lambda=e^{\Delta(\varphi+\beta)}, \quad \hat{\sigma}_{-}=\sigma_{-} \varsigma_{-}$,

shows that the actual dependence on parameters is

$H_{l \vec{k} r \sigma_{-}}(t)=\tilde{\phi}_{l \hat{\sigma}_{-}}(\tau)$

so that

$\partial_{\tau}^{2} \tilde{\phi}_{l \hat{\sigma}_{-}}+\frac{1}{\tau} \partial_{\tau} \tilde{\phi}_{l \hat{\sigma}_{-}}+\left[\frac{l^{2}}{(\Delta \tau)^{2}}+4 \hat{\sigma}_{-}\right] \tilde{\phi}_{l \hat{\sigma}_{-}}=0$.

The solutions have asymptotics

$\tilde{\phi}_{l \hat{\sigma}_{-}} \sim\left\{\begin{array}{ll}A_{+}|\tau|^{i \frac{l}{\Delta}}+A_{-}|\tau|^{-i \frac{l}{\Delta}} & l \neq 0 \\ A_{+} \log (|\tau|)+A_{-} & l=0\end{array}\right.$,

and we will be more concerned on the $l=0$ case as before.

\subsection{Eigenmodes on BOfrom covering space}

We now repeat the essential part of the analysis performed in the NBOcase. As in the NBOcase we use the wording wave function and not the eigenfunction because eigenfunctions for non scalar states require some constraints on polarizations which we do not impose.

\subsubsection{Spin O}

We start as usual with the Minkowskian wave function and we write only the dependence on $x^{+}$and $x^{-}$since all the other coordinates are spectators 


$$
\begin{aligned}
\psi_{k_{+} k_{-}}\left(x^{+}, x^{-}\right) & =e^{i\left(k_{+} x^{+}+k_{-} x^{-}\right)} \\
& =\psi_{k_{+} k_{-}}\left(t, \varphi, \sigma_{-}\right) \\
& =e^{i m t\left[e^{+\Delta(\varphi+\beta)}+\hat{\sigma}_{-} t e^{-\Delta(\varphi+\beta)}\right]} .
\end{aligned}
$$

We can compute the wave function on the orbifold by summing over all images

$$
\begin{aligned}
\Psi_{\left[k_{+} k_{-}\right]}\left(\left[x^{+}, x^{-}\right]\right) & =\sum_{n \in \mathbb{Z}} \psi_{k_{+} k_{-}}\left(\mathcal{K}^{n}\left(x^{+}, x^{-}\right)\right) \\
& =\sum_{n \in \mathbb{Z}} \psi_{k_{+} k_{-}}\left(x^{+} e^{2 \pi \Delta n}, x^{-} e^{-2 \pi \Delta n}\right) \\
& =\sum_{n \in \mathbb{Z}} e^{i\left\{\left[k_{+} e^{2 \pi \Delta n}\right] x^{+}+\left[k_{-} e^{-2 \pi \Delta n}\right] x^{-}\right\}} \\
& =\sum_{n \in \mathbb{Z}} \psi_{\mathcal{K}^{-n}\left(k_{+} k_{-}\right)}\left(x^{+}, x^{-}\right)
\end{aligned}
$$

where we write $\left[k_{+} k_{-}\right]$because the function depends on the equivalence class of $k_{+} k_{-}$only. The equivalence relation is given by

$k=\left(\begin{array}{l}k_{+} \\ k_{-}\end{array}\right) \equiv \mathcal{K}^{-n} k=\left(\begin{array}{c}k_{+} e^{2 \pi \Delta n} \\ k_{-} e^{-2 \pi \Delta n}\end{array}\right)$.

The previous equation explains the rationale for the parametrization (7.8) so that we can always choose a representative

$0 \leq \beta<2 \pi, \quad m \neq 0$,

or differently said $\beta \equiv \beta+2 \pi$ and therefore we can use the dual quantum number $l$ using a Fourier transform. Using the well adapted set of coordinates we can write the spin-0 wave function in a way to show the natural variables as

$$
\begin{aligned}
\Psi_{\left[k_{+} k_{-}\right]}\left(\left[x^{+}, x^{-}\right]\right) & =\sum_{n} e^{i \tau\left[\lambda e^{+2 \pi \Delta n}+\hat{\sigma}_{-} \lambda^{-1} e^{-2 \pi \Delta n}\right]} \\
& =\hat{\Psi}\left(\tau, \lambda, \hat{\sigma}_{-}\right) .
\end{aligned}
$$

Again the scalar eigenfunction has a unique equivalence class which mixes coordinates and momenta.

Now we use the basic trick used in Poisson resummation

$$
\begin{aligned}
\Psi_{\left[k_{+} k_{-}\right]}\left(\left[x^{+}, x^{-}\right]\right) \\
=\int_{-\infty}^{\infty} d s \delta_{P}(s) e^{i\left\{k_{+} x^{+} \Lambda^{s}+k_{-} x^{-} \Lambda^{-s}\right\}} \\
=\frac{1}{2 \pi} \sum_{l \in \mathbb{Z}}\left|\frac{k_{+} x^{+}}{k_{-} x^{-}}\right|^{-i \frac{l}{2 \Delta}} \\
\int_{-\infty}^{\infty} d s e^{i 2 \pi l s} e^{i \operatorname{sgn}\left(k_{+} x^{+}\right) \sqrt{\left|k_{+} k_{-} x^{+} x^{-}\right|}\left\{\Lambda^{s}+\sigma_{-} \varsigma_{-} \Lambda^{-s}\right\}} \\
=\frac{1}{2 \pi} \sum_{l \in \mathbb{Z}}\left(e^{\Delta(\varphi+\beta)}\right)^{-i \frac{l}{\Delta}}
\end{aligned}
$$

$$
\begin{aligned}
& \int_{-\infty}^{\infty} d s e^{i 2 \pi l s} e^{i m t\left\{\Lambda^{s}+\sigma_{-} \varsigma_{-} \Lambda^{-s}\right\}} \\
= & \frac{1}{2 \pi} \sum_{l \in \mathbb{Z}} e^{i l \beta}\left[e^{i l \varphi} \int_{-\infty}^{\infty} d s e^{-i 2 \pi l s} e^{i m t\left\{\Lambda^{s}+\sigma_{-} \varsigma_{-} \Lambda^{-s}\right\}}\right],
\end{aligned}
$$

where the last line represents the change of quantum number from $m \beta$ to $m l$ and allows us to identify

$\mathcal{N}_{B O} \tilde{\phi}_{l \hat{\sigma}_{-}}(\tau)=\frac{1}{2 \pi} \int_{-\infty}^{\infty} d s e^{-i 2 \pi l s} e^{i \tau\left\{\Lambda^{s}+\hat{\sigma}_{-} \Lambda^{-s}\right\}}$

where $\mathcal{N}_{B O}$ is a constant which depends on the normalization chosen for $\tilde{\phi}_{l} \hat{\sigma}_{-}$. This expression gives an integral representation of the o.d.e. solutions.

\subsubsection{Spin 2}

We start with the Minkowskian tensorial wave function where we suppress all directions but $x^{+}, x^{-}$and $x^{2}$ since all other directions behave as $x^{2}$. In this case differently from spin 0 we need to keep the dependence on $x^{2}$ since it is needed for non trivial physical polarizations since it enters in the transversality conditions. Explicitly

$$
\begin{aligned}
\mathcal{N}_{\text {BO }} \psi_{k S}^{[2]}\left(x^{+}, x^{-}, x^{2}\right) \\
=S_{\mu \nu} \mathrm{d} x^{\mu} \mathrm{d} x^{\nu} \psi_{k}(x) \\
=\left[S_{++}\left(\mathrm{d} x^{+}\right)^{2}+2 S_{+-} \mathrm{d} x^{+} \mathrm{d} x^{-}++2 S_{+2} \mathrm{~d} x^{+} \mathrm{d} x^{2}\right. \\
\left.\quad+S_{--}\left(\mathrm{d} x^{-}\right)^{2}+2 S_{-2} \mathrm{~d} x^{2} \mathrm{~d} x^{2}++S_{22}\left(\mathrm{~d} x^{2}\right)^{2}\right] \\
\quad e^{i\left(k_{+} x^{+}+k_{-} x^{-}+k_{2} x^{2}\right)}
\end{aligned}
$$

which we rewrite in orbifold coordinates

$$
\begin{aligned}
& \mathcal{N}_{B O} \psi_{k S}^{[2]}\left(t, \varphi, x^{2}, \sigma_{-}\right) \\
& =S_{\alpha \beta} \mathrm{d} x^{\alpha} \mathrm{d} x^{\beta} \psi_{k}(x) \\
& \quad\left[\mathrm{d} t^{2}\left(2 S_{+-} \sigma_{-}+S_{++} e^{2 \Delta \varphi}+S_{--} e^{-2 \Delta \varphi}\right)\right. \\
& \quad+2 \Delta t \mathrm{~d} t \mathrm{~d} \varphi\left(S_{++} e^{2 \Delta \varphi}-S_{--} e^{-2 \Delta \varphi}\right) \\
& \quad+\Delta^{2} t^{2} d \varphi^{2}\left(-2 S_{+-} \sigma_{-}+S_{++} e^{2 \Delta \varphi}+S_{--} e^{-2 \Delta \varphi}\right) \\
& \quad+2 \mathrm{~d} t \mathrm{~d} x^{2}\left(S_{-} e^{-\Delta \varphi} \sigma_{-}+S_{+2} e^{\Delta \varphi}\right) \\
& \quad+2 \Delta t \mathrm{~d} x^{2} d \varphi\left(S_{+2} e^{\Delta \varphi}-S_{-2} e^{-\Delta \varphi} \sigma_{-}\right) \\
& \left.\quad+\left(\mathrm{d} x^{2}\right)^{2} S_{22}\right] e^{i m t\left[e^{+\Delta(\varphi+\beta)}+\hat{\sigma}_{-} e^{-\Delta(\varphi+\beta)}\right]+i k_{2} x^{2}} .
\end{aligned}
$$


Now we define the tensor wave on the orbifold as a sum over all images as

$$
\begin{aligned}
\mathcal{N}_{B O} \Psi_{[k S]}^{[2]}([x]) & =\sum_{n}\left(\mathcal{K}^{n} d x\right) \cdot S \cdot\left(\mathcal{K}^{n} d x\right) \psi_{k}\left(\mathcal{K}^{n} x\right) \\
& =\sum_{n} d x \cdot\left(\mathcal{K}^{-n} S\right) \cdot d x \psi_{\mathcal{K}^{-n} k}(x)
\end{aligned}
$$

In the last line we have defined the induced action of the Killing vector on $(k, S)$ which can be explicitly written as

$\mathcal{K}^{-n}\left(\begin{array}{c}S_{++} \\ S_{+-} \\ S_{--} \\ S_{+2} \\ S_{-2} \\ S_{22}\end{array}\right)=\left(\begin{array}{c}e^{2 n \Delta \varphi} S_{++} \\ S_{+-} \\ e^{-2 n \Delta \varphi} S_{--} \\ e^{n \Delta \varphi} \Delta S_{+2} \\ e^{-n \Delta \varphi} S_{-2} \\ S_{22}\end{array}\right)$

and it amounts to a trivial scaling.

In orbifold coordinates computing the tensor wave on the orbifold simply amounts to sum over all the shifts $\varphi \rightarrow \varphi+$ $2 \pi n$. Then we have to give a close expression for the sum involving powers $e^{2 \pi \Delta n}$, explicitly we find

$$
\begin{aligned}
& \sum_{n}\left(e^{2 \pi \Delta n}\right)^{N} e^{i \tau\left[\lambda e^{+2 \pi \Delta n}+\hat{\sigma}_{-} \frac{1}{\lambda} e^{-2 \pi \Delta n}\right]} \\
& \quad=\left\{\begin{array}{ll}
{\left[\frac{1}{2}\left(\frac{1}{\lambda} \partial_{\tau}+\frac{1}{\tau} \partial_{\lambda}\right)\right]^{N} \hat{\Psi}\left(\tau, \lambda, \hat{\sigma}_{-}\right)} & N>0 \\
{\left[\frac{1}{2}\left(\lambda \partial_{\tau}-\frac{\lambda^{2}}{\tau} \partial_{\lambda}\right)\right]^{N} \hat{\Psi}\left(\tau, \lambda, \hat{\sigma}_{-}\right)} & N<0
\end{array},\right.
\end{aligned}
$$

where $\tau$ derivatives higher than 2 of $\tilde{\phi}_{l} \hat{\sigma}_{-}$can be reduced with the help of the differential equation (7.13).

We now have to identify the basic polaritazions on the orbifold. There are three basic observations. The quantum number $\beta$ is no longer a good quantum number on the orbifold and it is replaced by $l$. The relations among orbifold polarizations and Minkowski polarizations may depend on $\beta$ as long as the traceless and transversality conditions on the orbifold are independent of it. These conditions may be a linear combinations of the ones in Minkowski. Finally it seems reasonable to use the natural variable $\lambda=e^{\Delta(\varphi+\beta)}$. Therefore, as we could guess, we have:

$$
\begin{aligned}
& \mathcal{S}_{t t}=e^{-2 \Delta \beta} S_{++} \\
& \mathcal{S}_{t \varphi}=S_{+-} \\
& \mathcal{S}_{t 2}=e^{-\Delta \beta} S_{+2} \\
& \mathcal{S}_{\varphi \varphi}=e^{2 \Delta \beta} S_{--} \\
& \mathcal{S}_{\varphi 2}=e^{\Delta \beta} S_{-2} \\
& \mathcal{S}_{22}=S_{22}
\end{aligned}
$$

which can be trivially inverted as

$$
\begin{aligned}
& S_{++}=e^{2 \Delta \beta} \mathcal{S}_{t t} \\
& S_{+-}=\mathcal{S}_{t \varphi} \\
& S_{+2}=e^{\Delta \beta} \mathcal{S}_{t 2} \\
& S_{--}=e^{-2 \Delta \beta} \mathcal{S}_{\varphi \varphi} \\
& S_{-2}=e^{-\Delta \beta} \mathcal{S}_{\varphi 2} \\
& S_{22}=\mathcal{S}_{22} .
\end{aligned}
$$

When they are inserted into the trace condition they give

$\operatorname{tr}(S)=-2 \mathcal{S}_{t \varphi}+\mathcal{S}_{22}$,

while the transversality conditions become

$$
\begin{aligned}
& (k \cdot S)_{+}=-e^{\Delta \beta}\left(m \hat{\sigma}_{-} \sigma_{-} \mathcal{S}_{t t}+m \mathcal{S}_{t \varphi}-k_{2} \mathcal{S}_{t 2}\right) \\
& (k \cdot S)_{-}=-e^{-\Delta \beta}\left(m \hat{\sigma}_{-} \sigma_{-} \mathcal{S}_{t \varphi}+m \mathcal{S}_{\varphi \varphi}-k_{2} \mathcal{S}_{\varphi 2}\right) \\
& (k \cdot S)_{2}=-\left(m \hat{\sigma}_{-} \sigma_{-} \mathcal{S}_{t 2}+m \mathcal{S}_{\varphi 2}-k_{2} \mathcal{S}_{22}\right)
\end{aligned}
$$

which are independent of $\beta$ when set to zero.

The final expression for the wave function for the symmetric tensor on the orbifold reads

$$
\begin{aligned}
\Psi_{[k S]}^{[2]}([x]) & =\sum_{l \in \mathbb{Z}} e^{i l \beta}\left[S_{m l, t t}(\mathrm{~d} t)^{2}+2 S_{m l, t \varphi} \mathrm{d} t \mathrm{~d} \varphi+\right. \\
& +2 S_{m l, t 2} \mathrm{~d} t \mathrm{~d} x^{2}+S_{m l, \varphi \varphi}(\mathrm{d} \varphi)^{2} \\
& +2 S_{m l, \varphi 2} \mathrm{~d} \varphi \mathrm{d} x^{2}+ \\
& \left.+S_{m l, 22}\left(\mathrm{~d} x^{2}\right)^{2}\right]
\end{aligned}
$$

where the explicit expressions for the components are

$$
\begin{aligned}
& S_{m l, t t}=+\left[-\frac{\tilde{\phi}_{l \hat{\sigma}_{-}}(\tau) l \lambda^{\frac{i l}{\Delta}}\left(l \mathcal{S}_{t t}+i \Delta \mathcal{S}_{t t}+l \mathcal{S}_{\varphi \varphi}-i \Delta \mathcal{S}_{\varphi \varphi}\right)}{2 \Delta^{2}}\right] \frac{1}{\tau^{2}} \\
& +\left[\frac{1}{2 \Delta} \frac{d}{d \tau} \tilde{\phi}_{l \hat{\sigma}_{-}}(\tau) \lambda^{\frac{i l}{\Delta}}\left(i l \mathcal{S}_{t t}-i l \mathcal{S}_{\varphi \varphi}-\Delta \mathcal{S}_{t t}-\Delta \mathcal{S}_{\varphi \varphi}\right)\right] \frac{1}{\tau} \\
& +\left[\tilde{\phi}_{l \hat{\sigma}_{-}}(\tau) \lambda^{\frac{i l}{\Delta}}\left(\hat{\sigma}_{-} \mathcal{S}_{t t}+2 \sigma_{-} \mathcal{S}_{t \varphi}+\hat{\sigma}_{-} \mathcal{S}_{\varphi \varphi}\right)\right],
\end{aligned}
$$

and

$$
\begin{aligned}
S_{m l, t \varphi} & =+\left[-\frac{\tilde{\phi}_{l \hat{\sigma}_{-}}(\tau) l \lambda^{\frac{i l}{\Delta}}\left(l \mathcal{S}_{t t}+i \Delta \mathcal{S}_{t t}-l \mathcal{S}_{\varphi \varphi}+i \Delta \mathcal{S}_{\varphi \varphi}\right)}{2 \Delta m}\right] \frac{1}{\tau} \\
+ & {\left[\frac{\frac{d}{d \tau} \tilde{\phi}_{l \hat{\sigma}_{-}}(\tau) \lambda^{\frac{i l}{\Delta}}\left(i l \mathcal{S}_{t t}-\Delta \mathcal{S}_{t t}+i l \mathcal{S}_{\varphi \varphi}+\Delta \mathcal{S}_{\varphi \varphi)}\right)}{2 m}\right] } \\
+ & {\left[\frac{\Delta \hat{\sigma}_{-} \tilde{\phi}_{l \hat{\sigma}_{-}}(\tau) \lambda^{\frac{i l}{\Delta}}\left(\mathcal{S}_{t t}-\mathcal{S}_{\varphi \varphi}\right)}{m}\right] \tau, }
\end{aligned}
$$


and

$$
\begin{aligned}
& S_{m l, \varphi \varphi}=+\left[-\frac{1}{2 m^{2}} \tilde{\phi}_{l \hat{\sigma}_{-}}(\tau) l \lambda^{\frac{i l}{\Delta}}\right. \\
& \left.\quad\left(l\left(\mathcal{S}_{t t}+\mathcal{S}_{\varphi \varphi}\right)+i \Delta\left(\mathcal{S}_{t t}-\mathcal{S}_{\varphi \varphi}\right)\right)\right] \\
& +\left[\frac{1}{2 m^{2}} \Delta\left(\frac{d}{d \tau} \tilde{\phi}_{l \hat{\sigma}_{-}}(\tau)\right) \lambda^{\frac{i l}{\Delta}}\right. \\
& \left.\quad\left(i l \mathcal{S}_{t t}-i l \mathcal{S}_{\varphi \varphi}-\Delta \mathcal{S}_{t t}-\Delta \mathcal{S}_{\varphi \varphi}\right)\right] \tau \\
& +\left[\frac{1}{m^{2}} \Delta^{2} \tilde{\phi}_{l \hat{\sigma}_{-}}(\tau) \lambda^{\frac{i l}{\Delta}}\left(\hat{\sigma}_{-} \mathcal{S}_{t t}+\hat{\sigma}_{-} \mathcal{S}_{\varphi \varphi}-2 \sigma_{-} \mathcal{S}_{t \varphi}\right)\right] \tau^{2},
\end{aligned}
$$

and the effectively vector components in the orbifold directions

$$
\begin{aligned}
& S_{m l, t 2}=+\left[\frac{i}{2 \Delta} \tilde{\phi}_{l \hat{\sigma}_{-}}(\tau) l \lambda^{\frac{i l}{\Delta}}\left(\mathcal{S}_{t 2}-\mathcal{S}_{\varphi 2} \sigma_{-}\right)\right] \frac{1}{\tau} \\
& +\left[\frac{1}{2} \frac{d}{d \tau} \tilde{\phi}_{l \hat{\sigma}_{-}}(\tau) \lambda^{\frac{i l}{\Delta}}\left(\mathcal{S}_{t 2}+\mathcal{S}_{\varphi 2} \sigma_{-}\right)\right],
\end{aligned}
$$

and

$$
\begin{aligned}
& S_{m l, \varphi 2}=+\left[\frac{i}{2 m} \tilde{\phi}_{l \hat{\sigma}_{-}}(\tau) l \lambda^{\frac{i l}{\Delta}}\left(\mathcal{S}_{t 2}+\mathcal{S}_{\varphi 2} \sigma_{-}\right)\right] \\
& +\left[\frac{1}{2 m} \Delta\left(\frac{d}{d \tau} \tilde{\phi}_{l \hat{\sigma}_{-}}(\tau)\right) \lambda^{\frac{i l}{\Delta}}\left(\mathcal{S}_{t 2}-\mathcal{S}_{\varphi 2} \sigma_{-}\right)\right] \tau,
\end{aligned}
$$

and finally the effectively scalar component

$$
S_{m l, 22}=\mathcal{S}_{22} \tilde{\phi}_{l \hat{\sigma}_{-}}(\tau) \lambda^{i \frac{i l}{\Delta}} .
$$

7.4 Overlap of wave functions and their derivatives and a divergent three points String Amplitude

Now we consider some overlaps as done for the NBQ The connection between the overlaps on the orbifold and the sums of images remains unchanged when we change the Killing vector $\mathcal{K}$, hence we can limit ourselves to discuss the integrals on the orbifold space.

\subsubsection{Overlaps without derivatives}

Let us start with the simplest case of the overlap of $N$ scalar wave functions

$$
\begin{aligned}
I^{(N)}= & \left.\int_{\Omega} \mathrm{d}^{3} x \sqrt{-\operatorname{det} g} \prod_{i=1}^{N} \Psi_{\left[k_{+(i)} k_{-(i)}\right]}\left(\left[x^{+}, x^{-}, x^{2}\right]\right)\right) \\
= & \mathcal{N}_{B O}^{N} \sum_{\left\{l_{(i)}\right\} \in \mathbb{Z}^{N}} e^{i \sum_{i=1}^{N} l_{(i)} \beta_{(i)}} \\
& \int_{\Omega} \mathrm{d}^{3} x \sqrt{-\operatorname{det} g} \prod_{i=1}^{N} \phi_{l_{(i)} \hat{\sigma}_{-(i)} .}
\end{aligned}
$$

This is always a distribution since the problematic $l_{(*)}=0$ sector gives a divergence like $(\log (|t|))^{N}$ around zero. All other sectors have no issues because of the asymptotics (7.14).

\subsubsection{An overlap with two derivatives}

We consider in orbifold coordinates the overlap needed for the amplitude involving two tachyons and one massive state, i.e.

$$
\begin{gathered}
K=\int_{\Omega} \mathrm{d}^{3} x \sqrt{-\operatorname{det} g} g^{\alpha \beta} g^{\gamma \delta} \Psi_{\left[k_{(3)}, S_{(3)}\right] \alpha \gamma}^{[2]}([x]) \\
D_{\beta} \partial_{\delta} \Psi_{\left[k_{(2)}\right]}([x]) \Psi_{\left[k_{(1)}\right]}([x]) .
\end{gathered}
$$

Since we want to use the traceless condition we need to keep all momenta and polarizations and not only the ones along the orbifold, then we can write

$$
\begin{aligned}
K= & \int_{\Omega} \mathrm{d}^{3} x \sqrt{-\operatorname{det} g}\left[+\Psi_{\left[k_{(3)}, S_{(3)}\right] t t}^{[2]} \partial_{t}^{2} \Psi_{\left[k_{(2)}\right]}\right. \\
& -2\left(\frac{1}{\Delta t}\right)^{2} \Psi_{\left[k_{(3)}, S_{(3)}\right] t \varphi}^{[2]}\left(\partial_{t} \partial_{\varphi} \Psi_{\left[k_{(2)}\right]}-\frac{1}{t} \partial_{\varphi} \Psi_{\left[k_{(2)}\right]}\right) \\
& +\left(\frac{1}{\Delta t}\right)^{4} \Psi_{\left[k_{(3)}, S_{(3)}\right] \varphi \varphi}^{[2]}\left(\partial_{\varphi}^{2} \Psi_{\left[k_{(2)}\right]}-\Delta^{2} t \partial_{t} \Psi_{\left[k_{(2)}\right]}\right) \\
& -2 \Psi_{\left[k_{(3)}, S_{(3)}\right] t 2}^{[2]} \partial_{t} \partial_{2} \Psi_{\left[k_{(2)}\right]} \\
& +2\left(\frac{1}{\Delta t}\right)^{2} \Psi_{\left[k_{(3)}, S_{(3)}\right] \varphi 2}^{[2]} \partial_{\varphi} \partial_{2} \Psi_{\left[k_{(2)}\right]} \\
& \left.+\Psi_{\left[k_{(3)}, S_{(3)}\right] 22}^{[2]} \partial_{2}^{2} \Psi_{\left[k_{(2)}\right]}\right] \Psi_{\left[k_{(1)}\right]} .
\end{aligned}
$$

Now consider the behavior for $l_{(*)}=0$ for small $t$. All the $\partial_{\varphi}$ can be dropped since they lower a $l_{(2)}$. The leading contributions from spin 2 components are $S_{m l} t t \sim \frac{1}{t^{2}}$, $S_{m l \varphi \varphi}, S_{m l 22} \sim 1$ and $S_{m l t 2} \sim \frac{1}{t}$ therefore the leading $\frac{1}{t^{4}}$ reads

$$
\begin{aligned}
& K \sim \int_{t \sim 0} \mathrm{~d} t|t| \\
& \quad\left[-\frac{1}{2} \frac{d}{d \tau} \tilde{\phi}_{l \hat{\sigma}_{-}}\left(\mathcal{S}_{t t}+\mathcal{S}_{\varphi \varphi}\right) \frac{1}{\tau} \times \partial_{t}^{2} \Psi_{\left[k_{(2)}\right]}\right. \\
& \quad+\left(\frac{1}{\Delta t}\right)^{4} \times \frac{-\Delta^{2}}{2 m^{2}} \frac{d}{d \tau} \tilde{\phi}_{l \hat{\sigma}_{-}}\left(\mathcal{S}_{t t}+\mathcal{S}_{\varphi \varphi}\right) \tau \\
& \left.\quad \times\left(-\Delta^{2} t \partial_{t} \Psi_{\left[k_{(2)}\right]}\right)\right] \Psi_{\left[k_{(3)}\right]}
\end{aligned}
$$

In the limit of our interest $\left.\Psi_{[k]}\right|_{l=0} \sim \tilde{\phi}_{\left.l \hat{\sigma}_{-}\right|_{l=0} \sim \log (|t|)}$ then the two terms add together because of sign of the covariant derivative to give 
K

$$
\begin{gathered}
\sim \int_{t \sim 0} \mathrm{~d} t|t| \\
{\left[\left(\frac{1}{2}+\frac{1}{2}\right) \frac{\mathcal{S}_{t t}+\mathcal{S}_{\varphi \varphi}}{m^{4}} \frac{\log (|t|)}{t^{4}}+O\left(\frac{\log ^{2}(|t|)}{t}\right)\right],}
\end{gathered}
$$

which is divergent for the physical polarization $\mathcal{S}_{t}=\mathcal{S}_{\varphi \varphi}=$ $-\hat{\sigma}_{-} \sigma_{-} \mathcal{S}_{t \varphi}=-\frac{1}{2} \hat{\sigma}_{-} \sigma_{-} \mathcal{S}_{22}$.

Acknowledgements We thank Marco Billó and Domenico Orlando for discussions. The work is partially supported by the MIUR PRIN Contract 2015 MP2CX4 "Non-perturbative Aspects Of Gauge Theories And Strings".

Data Availability Statement This manuscript has no associated data or the data will not be deposited. [Author's comment: No data have been generated.]

Open Access This article is licensed under a Creative Commons Attribution 4.0 International License, which permits use, sharing, adaptation, distribution and reproduction in any medium or format, as long as you give appropriate credit to the original author(s) and the source, provide a link to the Creative Commons licence, and indicate if changes were made. The images or other third party material in this article are included in the article's Creative Commons licence, unless indicated otherwise in a credit line to the material. If material is not included in the article's Creative Commons licence and your intended use is not permitted by statutory regulation or exceeds the permitted use, you will need to obtain permission directly from the copyright holder. To view a copy of this licence, visit http://creativecomm ons.org/licenses/by/4.0/.

Funded by SCOAP ${ }^{3}$.

\section{A Complete tensor wave function in the NBO}

For the sake of completeness we report the expression of the full NBOtensor wave function $\left(=\frac{l}{k_{+}}\right)$:

$$
\begin{aligned}
& \left(\begin{array}{l}
S_{u u} \\
S_{u v} \\
S_{u z} \\
S_{u i} \\
S_{v v} \\
S_{v z} \\
S_{v i} \\
S_{z z} \\
S_{z i} \\
S_{i i}
\end{array}\right)=\mathcal{S}_{u u}\left(\begin{array}{c}
1 \\
0 \\
0 \\
0 \\
0 \\
0 \\
0 \\
0 \\
0 \\
0
\end{array}\right) \phi_{\left\{k_{+} l \vec{k} r\right\}}+\mathcal{S}_{u v}\left(\begin{array}{c}
\frac{i}{k_{+} u}+\frac{L^{2}}{\Delta^{2} u^{2}} \\
1 \\
L \\
0 \\
0 \\
0 \\
0 \\
0 \\
0 \\
0
\end{array}\right) \phi_{\left\{k_{+} l \vec{k} r\right\}} \\
& +\mathcal{S}_{u z}\left(\begin{array}{c}
\frac{2 L}{\Delta u} \\
0 \\
\Delta u \\
0 \\
0 \\
0 \\
0 \\
0 \\
0 \\
0
\end{array}\right) \phi_{\left\{k_{+} l \vec{k} r\right\}}+\mathcal{S}_{u i}\left(\begin{array}{c}
0 \\
0 \\
0 \\
1 \\
0 \\
0 \\
0 \\
0 \\
0 \\
0
\end{array}\right) \phi_{\left\{k_{+} l \vec{k} r\right\}}
\end{aligned}
$$

$$
+\mathcal{S}_{v v}\left(\begin{array}{c}
-\frac{3}{4 k_{+}^{2} u^{2}}+\frac{3 i L^{2}}{2 \Delta^{2} k_{+} u^{3}}+\frac{L^{4}}{4 \Delta^{4} u^{4}} \\
\frac{i}{2 k_{+} u}+\frac{L^{2}}{2 \Delta^{2} u^{2}} \\
\frac{3 i L}{2 k_{+} u}+\frac{L^{3}}{2 \Delta^{2} u^{2}} \\
0 \\
1 \\
L \\
0 \\
\frac{i \Delta^{2} u}{k_{+}}+L^{2} \\
0 \\
0
\end{array}\right) \phi_{\left\{k_{+} l \vec{k} r\right\}}
$$$$
+\mathcal{S}_{v z}\left(\begin{array}{c}
\frac{3 i L}{\Delta k_{+} u^{2}}+\frac{L^{3}}{\Delta^{3} u^{3}} \\
\frac{L}{\Delta u} \\
\frac{3 L^{2}}{2 \Delta u}+\frac{3 i \Delta}{2 k_{+}} \\
0 \\
0 \\
\Delta u \\
0 \\
2 \Delta u \\
0 \\
0
\end{array}\right) \phi_{\left\{k_{+} l \vec{k} r\right\}}
$$$$
+\mathcal{S}_{v i}\left(\begin{array}{c}
0 \\
0 \\
0 \\
\frac{i}{2 k_{+} u}+\frac{L^{2}}{2 \Delta^{2} u^{2}} \\
0 \\
0 \\
1 \\
0 \\
L \\
0
\end{array}\right) \phi_{\left\{k_{+} l \vec{k} r\right\}}
$$$$
+\mathcal{S}_{z z}\left(\begin{array}{c}
\frac{i}{k_{+} u}+\frac{L^{2}}{\Delta^{2} u^{2}} \\
0 \\
L \\
0 \\
0 \\
0 \\
0 \\
\Delta^{2} u^{2} \\
0 \\
0
\end{array}\right) \phi_{\left\{k_{+} l \vec{k} r\right\}}
$$ 


$$
\begin{aligned}
& +\mathcal{S}_{z i}\left(\begin{array}{c}
0 \\
0 \\
0 \\
\frac{L}{\Delta u} \\
0 \\
0 \\
0 \\
0 \\
\Delta u \\
0
\end{array}\right) \phi_{\left\{k_{+} l \vec{k} r\right\}} \\
& +\mathcal{S}_{i j}\left(\begin{array}{c}
0 \\
0 \\
0 \\
0 \\
0 \\
0 \\
0 \\
0 \\
0 \\
\delta_{i j}
\end{array}\right) \phi_{\left\{k_{+} l \vec{k} r\right\}} .
\end{aligned}
$$

\section{B Complete overlap with two derivatives in the NBO}

For the sake of completeness we report the full expression of the overlap with two derivatives considered in the main text which corresponds to the colour ordered amplitude of two tachyons and one level 2 massive state:

$$
\begin{aligned}
K= & \mathcal{N}^{2} \int \mathrm{d}^{D} x \sqrt{-\operatorname{det} g} \\
& {\left[\mathfrak{s}^{(-3)}\left(\left\{k_{(i)+} l_{(i)} \vec{k}_{(i)} r_{(i)}\right\}_{i=1,2,3},\{\mathcal{S}\}\right) u^{-3}\right.} \\
& +\mathfrak{s}^{(-2)}\left(\left\{k_{(i)+} l_{(i)} \vec{k}_{(i)} r_{(i)}\right\}_{i=1,2,3},\{\mathcal{S}\}\right) u^{-2} \\
& +\mathfrak{s}^{(-1)}\left(\left\{k_{(i)+} l_{(i)} \vec{k}_{(i)} r_{(i)}\right\}_{i=1,2,3},\{\mathcal{S}\}\right) u^{-1} \\
& +\mathfrak{s}^{(0)}\left(\left\{k_{(i)+} l_{(i)} \vec{k}_{(i)} r_{(i)}\right\}_{i=1,2,3},\{\mathcal{S}\}\right) \\
& \left.+\mathfrak{s}^{(1)}\left(\left\{k_{(i)}+l_{(i)} \vec{k}_{(i)} r_{(i)}\right\}_{i=1,2,3},\{\mathcal{S}\}\right) u\right] \\
& \prod_{i=1}^{3} \phi_{\left\{k_{(i)+} l_{(i)} \vec{k}_{(i)} r_{(i)}\right\}}
\end{aligned}
$$

where:

$$
\begin{aligned}
& \mathfrak{s}^{(-3)}=\left(-\frac{k_{(2)+}^{4} l_{(3)}{ }^{4}-4 k_{(2)+}^{3} k_{(3)+} l_{(2)} l_{(3)}{ }^{3}}{4 k_{(2)+}^{2} k_{(3)+}^{4} \Delta^{3}}\right. \\
& \left.-\frac{6 k_{(2)+}^{2} k_{(3)+}^{2} l_{(2)}^{2} l_{(3)}^{2}+k_{(3)+}^{4} l_{(2)}{ }^{4}}{4 k_{(2)+}^{2} k_{(3)+}^{4} \Delta^{3}}\right) \mathcal{S}_{v v}, \\
& \mathfrak{s}^{(-2)} \\
& =\left(-\frac{i\left(3 k_{(2)+}^{2} k_{(3)+} l_{(3)}{ }^{2}+3 k_{(2)+}^{3} l_{(3)}{ }^{2}-4 k_{(2)+} k_{(3)+}^{2} l_{(2)} l_{(3)}-6 k_{(2)+}^{2} k_{(3)+} l_{(2)} l_{(3)}\right)}{2 k_{(2)+k_{(3)+}^{3}} \Delta}\right.
\end{aligned}
$$

$$
\begin{aligned}
& \left.+\frac{-i\left(+3 k_{(3)+}^{3} l_{(2)}^{2}+3 k_{(2)+} k_{(3)+}^{2} l_{(2)}^{2}\right)}{2 k_{(2)}+k_{(3)+}^{3} \Delta}\right) \mathcal{S}_{v v} \\
& +\left(-\frac{l_{(3)}\left(k_{(2)+}^{2} l_{(3)}^{2}-3 k_{(2)}+k_{(3)}+l_{(2)} l_{(3)}+3 k_{(3)+}^{2} l_{(2)}^{2}\right)}{k_{(3)+}^{3} \Delta^{2}}\right) \mathcal{S}_{v z}, \\
& \mathfrak{s}^{(-1)}=\left(-\frac{\left(k_{(2)}+l_{(3)}-k_{(3)}+l_{(2)}\right)^{2}}{k_{(3)+}^{2} \Delta}\right) \mathcal{S}_{u v} \\
& +\left(-\frac{2 k_{(2)+}^{2} l_{(3)}^{2}\left(r_{(2)}+\vec{k}_{(2)}^{2}\right)+2 k_{(3)+}^{2} l_{(2)}^{2}\left(r_{(2)}+\vec{k}_{(2)}^{2}\right)-8 k_{(2)+}^{3} k_{(3)}+l_{(2)} l_{(3)}}{4 k_{(2)+}^{2} k_{(3)+}^{2} \Delta}\right. \\
& \left.-\frac{-3 k_{(2)+}^{2} k_{(3)+}^{2} \Delta^{2}-6 k_{(2)+}^{3} k_{(3)+} \Delta^{2}-3 k_{(2)+}^{4} \Delta^{2}}{4 k_{(2)+}^{2} k_{(3)+}^{2} \Delta}\right) \mathcal{S}_{v v} \\
& +\left(-\frac{i\left(3 k_{(2)+} k_{(3)+} l_{(3)}+3 k_{(2)+}^{2} l_{(3)}-2 k_{(3)+}^{2} l_{(2)}-3 k_{(2)+} k_{(3)+} l_{(2)}\right)}{k_{(3)+}^{2}}\right) \mathcal{S}_{v z} \\
& +\left(\frac{k_{(2) i} l_{(3)}\left(k_{(2)}+l_{(3)}-2 k_{(3)}+l_{(2)}\right)}{k_{(3)+}^{2} \Delta}\right) \mathcal{S}_{v i} \\
& +\left(-\frac{\left(k_{(2)}+l_{(3)}-k_{(3)+} l_{(2)}\right)^{2}}{k_{(3)+}^{2} \Delta}\right) \mathcal{S}_{z z}, \\
& \mathfrak{s}^{(0)}=\left(-\frac{i k_{(2)+}\left(k_{(3)+}+k_{(2)+}\right) \Delta}{k_{(3)+}}\right) \mathcal{S}_{u v} \\
& +\left(-\frac{2 k_{(2)}+\left(k_{(2)}+l_{(3)}-k_{(3)}+l_{(2)}\right)}{k_{(3)+}}\right) \mathcal{S}_{u z} \\
& +\left(-\frac{i\left(k_{(3)+}+k_{(2)+}\right) \Delta\left(r_{(2)}+\vec{k}_{(2)}^{2}\right)}{2 k_{(2)}+k_{(3)+}}\right) \mathcal{S}_{v v} \\
& +\left(-\frac{l_{(3)}\left(r_{(2)}+\vec{k}_{(2)}^{2}\right)-2 k_{(2)}+k_{(3)}+l_{(2)}}{k_{(3)+}}\right) \mathcal{S}_{v z} \\
& +\left(\frac{i k_{(2) i} k_{(2)}+\Delta}{k_{(3)+}}\right) \mathcal{S}_{v i} \\
& +\left(-\frac{i k_{(2)+}\left(k_{(3)+}+k_{(2)+}\right) \Delta}{k_{(3)+}}\right) \mathcal{S}_{z z} \\
& +\left(\frac{2 k_{(2) i}\left(k_{(2)}+l_{(3)}-k_{(3)}+l_{(2)}\right)}{k_{(3)+}}\right) \mathcal{S}_{z i}, \\
& \mathfrak{s}^{(1)}=\left(-k_{(2)+}^{2} \Delta\right) \mathcal{S}_{u u} \\
& +\left(-\Delta\left(r_{(2)}+\vec{k}_{(2)}^{2}\right)\right) \mathcal{S}_{u v} \\
& +\left(2 k_{(2) i} k_{(2)}+\Delta\right) \mathcal{S}_{u i} \\
& +\left(-\frac{\Delta\left(r_{(2)}+\vec{k}_{(2)}^{2}\right)^{2}}{4 k_{(2)+}^{2}}\right) \mathcal{S}_{v v} \\
& +\left(2 k_{(2) i} k_{(2)}+\Delta\right) \mathcal{S}_{v i} \\
& +\left(-k_{(2) i} k_{(2) j} \Delta\right) \mathcal{S}_{i j} \text {. }
\end{aligned}
$$

\section{References}

1. B. Craps, D. Kutasov, G. Rajesh, String propagation in the presence of cosmological singularities. JHEP 06, 053 (2002)

2. H. Liu, G.W. Moore, N. Seiberg, Strings in a time dependent orbifold. JHEP 06, 045 (2002)

3. H. Liu, G.W. Moore, N. Seiberg, Strings in time dependent orbifolds. JHEP 10, 031 (2002)

4. L. Cornalba, M.S. Costa, Time dependent orbifolds and string cosmology. Fortsch. Phys. 52, 145-199 (2004)

5. B. Craps, Big Bang Models in String Theory. Class. Quant. Grav. 23, S849-S881 (2006)

6. M. Berkooz, B. Craps, D. Kutasov, G. Rajesh, Comments on cosmological singularities in string theory. JHEP 03, 031 (2003)

7. G.T. Horowitz, J. Polchinski, Instability of space-like and null orbifold singularities. Phys. Rev. D 66, 103512 (2002)

8. B. Pioline, M. Berkooz, Strings in an electric field, and the milne universe. JCAP 11, 007 (2003) 
9. J.M. Figueroa-O'Farrill, J. Simon, Generalized supersymmetric fluxbranes. JHEP 12, 011 (2001)

10. L. Cornalba, M.S. Costa, A new cosmological scenario in string theory. Phys. Rev. D 66, 066001 (2002)

11. G.T. Horowitz, A.R. Steif, Singular string solutions with nonsingular initial data. Phys. Lett. B 258, 91-96 (1991)

12. J. Khoury, B.A. Ovrut, N. Seiberg, P.J. Steinhardt, N. Turok, From big crunch to big bang. Phys. Rev. D 65, 086007 (2002)

13. A. Arduino, R. Finotello, I. Pesando, Work in progress

14. R. Jackiw, D.N. Kabat, M. Ortiz, Electromagnetic fields of a massless particle and the eikonal. Phys. Lett. B 277, 148-152 (1992)
15. G. 't Hooft, Graviton dominance in ultrahigh-energy scattering. Phys. Lett. B 198, 61-63 (1987)

16. M. Soldate, Partial wave unitarity and closed string amplitudes. Phys. Lett. B 186, 321-327 (1987)

17. D. Amati, M. Ciafaloni, G. Veneziano, Superstring collisions at planckian energies. Phys. Lett. B 197, 81 (1987)

18. W. Black, C. Monni, High energy string-brane scattering for massive states. Nucl. Phys. B 859, 299-320 (2012) 